\title{
WestVirginiaUniversity
}

THE RESEARCH REPOSITORY @ WVU

Graduate Theses, Dissertations, and Problem Reports

2011

\section{Role of Inhibition in Binaural Processing}

Jesse M. Thompson

West Virginia University

Follow this and additional works at: https://researchrepository.wvu.edu/etd

\section{Recommended Citation}

Thompson, Jesse M., "Role of Inhibition in Binaural Processing" (2011). Graduate Theses, Dissertations, and Problem Reports. 4804.

https://researchrepository.wvu.edu/etd/4804

This Dissertation is protected by copyright and/or related rights. It has been brought to you by the The Research Repository @ WVU with permission from the rights-holder(s). You are free to use this Dissertation in any way that is permitted by the copyright and related rights legislation that applies to your use. For other uses you must obtain permission from the rights-holder(s) directly, unless additional rights are indicated by a Creative Commons license in the record and/ or on the work itself. This Dissertation has been accepted for inclusion in WVU Graduate Theses, Dissertations, and Problem Reports collection by an authorized administrator of The Research Repository @ WVU.

For more information, please contact researchrepository@mail.wvu.edu. 
Role of Inhibition in Binaural Processing

Jesse M. Thompson

\begin{abstract}
Dissertation submitted to the School of Medicine at West Virginia University In partial fulfillment of the requirements for the degree of

Doctor of Philosophy In Cellular and Integrative Physiology
\end{abstract}

George A. Spirou, Ph.D. Chair

Albert S. Berrebi, Ph.D.

Kevin C. Daly, Ph.D.

Stan M. Hileman, Ph.D.

James W. Lewis, PhD

Department of Physiology and Pharmacology

Morgantown, West Virginia

2011

Keywords: Sound localization, Temporal processing 


\section{Abstract \\ Role of Inhibition in Binaural Processing}

Jesse M. Thompson

The medial and lateral superior olives (MSO, LSO) are the lowest order cell groups in the mammalian auditory circuit to receive massive binaural input. The MSO functions in part to encode interaural time differences (ITD), the predominant cue for localization of low frequency sounds. Binaural inputs to the MSO consist of excitatory projections from the cochlear nuclei $(\mathrm{CN})$ and inhibitory projections from both the medial nucleus of the trapezoid body (MNTB) and lateral nucleus of the trapezoid body (LNTB). The interaction of excitatory and inhibitory currents within an MSO cell's soma and dendrites over the backdrop of its intrinsic ionic conductances imbues ITD sensitivity to these neurons. Lloyd Jeffress proposed a coincidence detection circuit in which arrays of neurons receive sub-threshold excitatory inputs via delay lines that represent sound location as a place code of activity patterns within the cell group (Jeffress, 1948). The Jeffress place code model later found a neural instantiation in the MSO. Recent in vivo (McAlpine et al., 2001; Brand et al., 2002) studies have shown that peak discharge rates do not fall within the ecological range as the Jeffress model predicts but instead ITD is coded by changes in discharge rate. The timing of inhibition relative to excitation modulates the discharge rates of MSO cells (Brand et al., 2002; Chirila et al., 2007); however, the details of this circuit, such as the onset time of inhibition, are not well known. Although the MNTB and LNTB have been investigated in vivo and in vitro, they have not been well characterized with respect to their function in ITD processing in larger mammals. Additionally, inhibition is modulated by anesthesia and confounds in vivo experiments that examine the careful interplay of excitatory and inhibitory effects in the MSO. For this reason, these physiological experiments were performed on decerebrate unanaesthetized animals. Further investigation of the anatomical organization of inhibitory inputs was carried out as the basis for a comprehensive model of the MSO that incorporates the effects of binaural inhibiting projections to MSO neurons.

Unbiased stereological counts of the MNTB, MSO and subdivisions of the LNTB showed that the MSO and MNTB contain approximately the same number of cells. The main $(\mathrm{m})$ LNTB, posteroventral $(\mathrm{pv})$ LNTB and the hilus $(\mathrm{h})$ LNTB are estimated to contain 3800,1400 , and 200 neurons respectively. Tonotopic organization of the MNTB and MSO show that in the low frequency area, MSO cells outnumber MNTB cells 2 to 1 , suggesting a divergent innervation of the MSO from the MNTB. Injection of the retrograde tracer, biotinylated dextrane amine, in the MSO, labeled cells in the MNTB, pvLNTB and mLNTB and defines the important role that these sub-nuclei, and in particular the pvLNTB, have in ITD coding. Computational modeling of a single MSO cell suggests that when two sources of inhibition temporally frame excitation the coincidence detection window is refined and less sensitive to temporal fluctuations that otherwise might degrade ITD sensitivity. Finally, physiological properties of MNTB cells reveal a heterogeneous population of responses and less precise temporal coding than are found in their inputs, globular bushy cells. 
Acknowledgments

Many people were necessary in the completion of this dissertation and I would like to thank them all, as a group, in case I fail to thank them in the following acknowledgements. First I would like to thank my advisor, Dr. George Spirou. His approach to scientific problems and mentoring may not be the most expeditious but it is thorough. l'd like to thank him for his "see one, do one, teach one" mantra which forced me to learn a lot on my own but was beneficial in that a deep understanding and appreciation of an entire problem is gained.

I would like to thank my current committee members, Dr. Albert Berrebi, Dr. Kevin Daly, Dr. Stan Hileman and Dr. James Lewis. Your feedback and advice have been valuable and your patience and flexibility in these last difficult months has been greatly appreciated. I would also like to thank former committee members, Dr. Paul Brown and Dr. Ronald Millecchia for their contributions in the sounds booth.

Shout out to the Spirou lab, in no particular order. Thank you, Kevin Rowland (and Darcy) for housing me for the first couple months of my graduate career. Kevin you were instrumental in teaching me how to patch clamp. l'd like to thank Doug for our extracurricular fishing trips, Florin for the reintroduction of soccer into my adult life and professional advice. I'd like to thank Glen "Nomnom" Marrs for his childlike humor and his questionable scientific writing advice. Special thanks to Brian Pope for teaching me the tedious lab skills of tissue-prep and brain reconstruction. Your musical tastes are questionable but your beer palate spot on.

None of this would be possible without my parents. In my formative years, my exposure to the outdoors and your taxonomic knowledge of trees and insects sparked an interest in the biological world around me. This need, to know how things work, first directed me into computer engineering and later into neuroscience. Finally l'd like to thank Lori for all of her love, support and patience with George. Thanks for all of the fantastic meals you have cooked and helped me cook over the years. These meals and our time together acted as a safety valve to the pressure cooker that is grad school. Thank you for pushing me back to lab to write when I wanted to stay on the couch and crip-out. With these words, I thank thee. 


\section{Table of Contents}

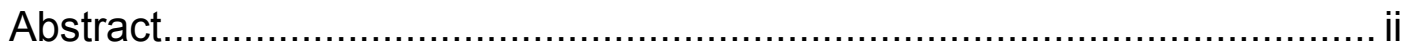

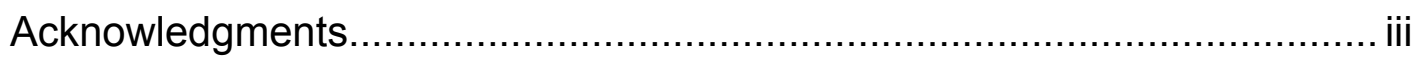

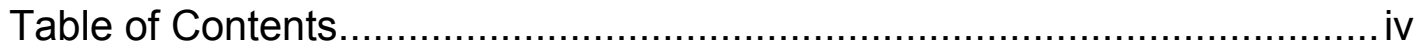

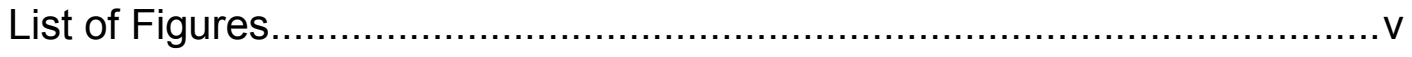

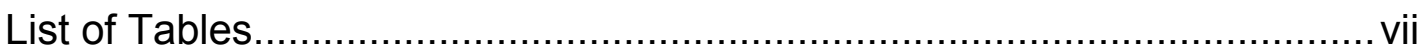

Glossary of Abbreviations.................................................................ii

1. Aims

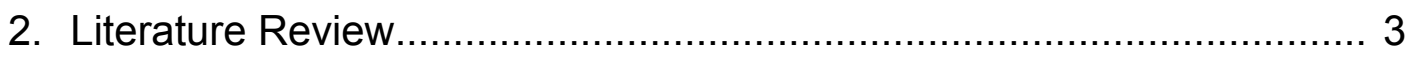

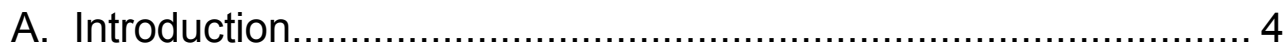

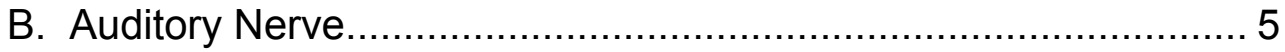

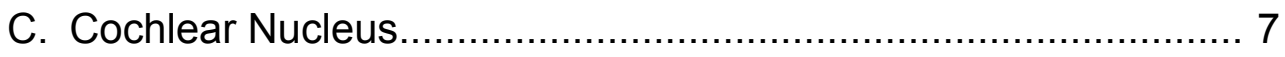

i. Cochlear Nucleus Anatomy................................................ 8

ii. Cochlear Nucleus Physiology......................................... 10

D. Superior Olivary Complex.................................................... 14

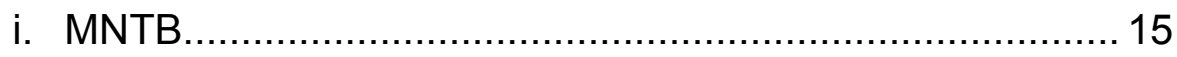

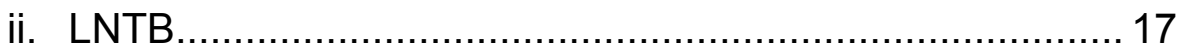

iii. MSO

a. MSO Anatomy ............................................... 20

b. MSO Physiology.................................................. 23

3. Binaural Inhibition in the Medial Superior Olive ................................. 33

4. Neural Encoding of Sound in the MNTB........................................... 86

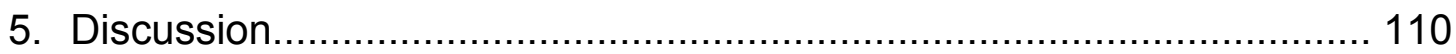

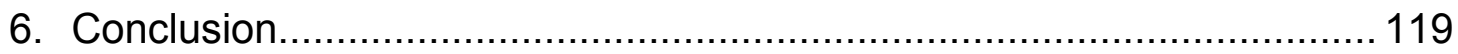

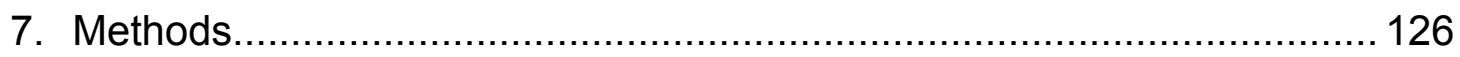

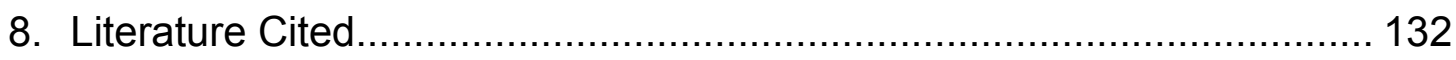

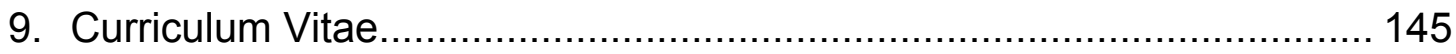




\section{List of Figures}

Literature review

Figure 2-1. Physical characteristics of sound relevant to the duplex theory of sound localization......................................................... 28

Figure 2-2. Jeffress coincidence detection circuit........................... 29

Figure 2-3. Phase locked and primary-like (PL) peri-stimulus time histograms

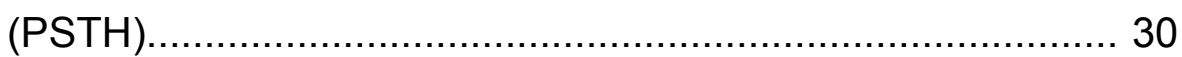

Figure 2-4. Schematic diagram of the cochlear nucleus (CN) .............. 31

Figure $2-5 . \quad$ The superior olivary complex..................................... 32

Binaural Inhibition in Medial Superior Olive

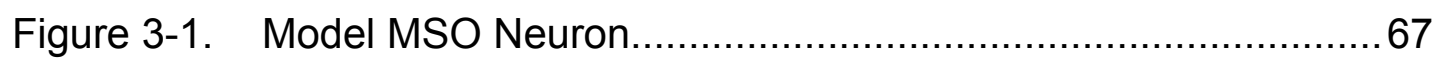

Figure 3-2. Cell density varies across tonotopic location in MNTB and MSO.................................................. 68

Figure 3-3. Retrograde labeling of inputs to the MSO

Figure 3-4. Distribution of retrogradely labeled cells in MNTB and LNTB from focal MSO injection site....................................... 70

Figure 3-5. Composite IPSPs from inhibitory cell groups based on location and axonal conduction velocity.................................... 72

Figure 3-6. ITD curves generated with bilateral inputs, EE model

Figure 3-7. ITD curves generated with bilateral excitatory and unilateral inhibitory inputs, EIE model. 75

Figure 3-8. ITD curves generated with bilateral excitatory and bilateral inhibitory inputs, EIEl model..................................... 76

Figure 3-9. EIEl model for a range of MNTB delays............................ 78

Figure 3-10. Variation in conductance and number of inputs demonstrate advantages in EIEI model compared w/ EIE and EE model... 80

Figure 3-11. EIE models generate ITD curves with steeper slopes. 81 
Neural encoding of sound in the MNTB

Figure 4-1. 3D reconstruction of the brainstem and SOC .......................95

Figure 4-2. Phase-locked responses for low frequency units................... 96

Figure 4-3. Phase-locked and primary-like with notch PSTHs................ 97

Figure 4-4. Primary-like with multiple notches and sustained-chopper

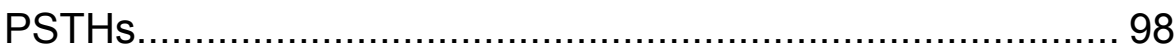

Figure 4-5. Transient-chopper and primary-like with multiple notches

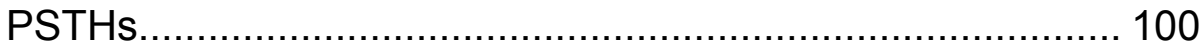

Figure 4-6. Primary-like with notch PSTH............................................. 101

Figure 4-7. First spike latency for tone and click stimuli......................... 103

Figure 4-8. Spontaneous and max sustained discharge rates..................104

Figure 4-9. Phase-locking to $500 \mathrm{~Hz}$ pure tone.....................................105

Figure 4-10. Phase-locking in low CF MNTB units................................. 106

Figure 4-11. Temporal modulation transfer functions (tMTFs) ...................108

Figure 4-12. Phase PSTHs for SAM tones............................................ 109

Conclusion

Figure 6-1. ITD curves of the MSO and IC....................................... 125

Methods

Figure 7-1. Landmarks of the MNTB on the floor of the fourth ventricle 


\section{List of Tables}

Binaural Inhibition in Medial Superior Olive

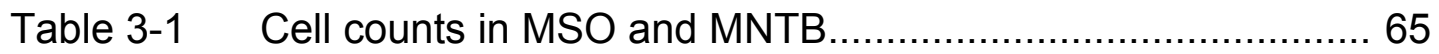

Table 3-2 Number of cells labeled retrogradely via BDA........................ 66

Neural encoding of sound in the MNTB

Table 4-1 Response characteristics of MNTB cells.............................. 102 
Glossary of Abbreviations

$\begin{array}{ll}\text { ABRs } & \text { Auditory brainstem responses } \\ \text { AM } & \text { Amplitude modulation } \\ \text { ANFs } & \text { Auditory nerve fibers } \\ \text { AVCN } & \text { Anteroventral cochlear nucleus } \\ \text { AP } & \text { Action potential } \\ \text { BDA } & \text { Biotinylated dextran amine (retrograde tracer) } \\ \text { BV } & \text { Biological variation } \\ \text { CD } & \text { Characteristic delay } \\ \text { CE } & \text { Coefficient of error } \\ \text { CF } & \text { Characteristic frequency } \\ \text { CMF } & \text { Characteristic modulation frequency } \\ \text { CN } & \text { Cochlear nucleus } \\ \text { CV } & \text { Coefficient of variation } \\ \text { DCN } & \text { Dorsal cochlear nucleus } \\ \text { EE } & \text { Excitatory excitatory } \\ \text { EIE } & \text { Excitatory inhibitory excitatory } \\ \text { EIEI } & \text { Excitatory inhibitory excitatory inhibitory } \\ \text { EPSPs } & \text { Excitatory post-synaptic potentials } \\ \text { EM } & \text { Electron microscopy } \\ \text { FSL } & \text { First spike latency } \\ \text { GABA } & \text { Gamma-aminobutyric acid } \\ \text { GBCs } & \text { Globular bushy cells } \\ \text { hLNTB } & \text { Hilus of the lateral nucleus of the trapezoid body } \\ \text { HRP } & \text { Horse radish peroxidase } \\ \text { IC } & \text { Inferior colliculus } \\ \text { ILD } & \text { Interaural level difference } \\ \text { IPD } & \text { Interaural phase difference } \\ \text { IPSPs } & \text { Inhibitory post-synaptic potentials } \\ \text { ISIH } & \text { Interspike interval histogram } \\ \text { ITD } & \text { Interaural temporal delay } \\ \text { LCZ } & \text { Large cell zone } \\ \text { LL } & \text { Lateral lemniscus } \\ \text { LNTB } & \text { Lateral nucleus of the trapezoid body } \\ \text { LSO } & \text { Lateral superior olive } \\ \text { MF } & \text { Modulation frequency } \\ \text { mLNTB } & \text { Main lateral nucleus of the trapezoid body } \\ \text { MNTB } & \text { Medial nucleus of the trapezoid body } \\ \text { MPO } & \text { Medial periolivary nucleus } \\ \text { MSO } & \text { Medial superior olive } \\ \text { NL } & \text { Nucleus laminaris } \\ \text { PL } & \text { Primary-like } \\ \text { PLN } & \text { Primary-like with notch } \\ & \end{array}$


PSTH Peri-stimulus time histogram

pvLNTB Posteroventral lateral nucleus of the trapezoid body

PVCN Posterior ventral cochlear nucleus

R Synchronization coefficient

SAM Sinusoidally amplitude modulated

SBCs Spherical bushy cells

SCR Spherical cell region

SCZ Small cell zone

SD Standard deviation

SOC Superior olivary complex

SR Spontaneous rate

TDT Tucker-Davis Technologies

tMTF Temporal modulation transfer function

VCN Ventral cochlear nucleus

VDL Visual detection level

VS Vector strength 


\section{Aims}

AIM 1 is to count the number of cells in the nuclei that make up ITD circuitry and determine the contributions that MNTB and LNTB make in local projections to MSO neurons. Estimates, using modern stereological techniques, of the number of cells in the MNTB, MSO and LNTB are absent in mammals sensitive to low frequency. The MNTB and LNTB do have projections terminating in the MSO (Cant and Hyson, 1992; Kuwabara and Zook, 1992; Smith et al., 1998). However, the topography of projections to particular regions of the MSO is unknown. The LNTB is composed of three subnuclei and it is unknown which of these subnuclei have significant inputs to the MSO. Numbers of cells, their patterns of projection and distances to postsynaptic targets in the MSO serve as a basis for understanding the ITD coding circuitry and creating computational models. I hypothesize that we will observe a tonotopic distribution of inputs from the MNTB and that the pVLNTB and mLNTB will provide substantial innervation to the MSO.

AIM 2 is to characterize responses of MNTB cells to sound in the absence of anesthesia, and to quantify their phase-locking ability. MNTB cells receive excitation from globular bushy cells via the calyx of Held and inhibitory inputs from unknown sources. Previously it has been argued that the MNTB functions simply as a signal-inverter converting temporally precise phase-locked excitation into temporally precise phase-locked inhibition; however, recent studies have shown that discharge of the calyx does not always lead to action potential generation in the MNTB cell, indicating a more complicated transfer function (KoppScheinpflug et al., 2003). I hypothesize that inhibitory inputs will be unmasked, generating a greater variety of peri-stimulus time histogram (PSTH) patterns and better phase locking than are found in anesthetized animals. Additionally, evidence of inhibition will be illustrated via an increase in spontaneous discharge rates and temporally complex phase-locking to pure tones and sinusoidally amplitude modulated tones (SAMs).

AIM 3 is to create a computational model of an MSO cell that incorporates data gathered from Aims $1-2$, and more specifically to examine the effect that onset time of two sources of 
inhibition and excitation have on ITD coding. Current models have established that a single source of inhibition preceding excitation is capable of shifting the peak discharge rate 100$200 \mu$ s. A linear summation model has examined ITD curves in the context of a system having two sources of inhibition, but absent from this model was anatomically accurate cell morphology and ion channels known of MSO cells. I propose that an additional source of inhibition, as suggested by connectivity of the MSO, will modify the ITD curve in ways not possible with a single source of inhibition. By parameterizing the temporal onset space of IPSPs and EPSPs, our computational model will allow us to explore and rule out onset times incongruent with ITD coding in small mammals utilizing a rate code, and provide insight to how a second source of inhibition would be utilized in larger mammals whose model of sound localization is currently under debate. 
2. Literature Review 


\section{A. Introduction}

The ability to localize sound is an amazing feat performed by many invertebrates and most vertebrates; it is essential for survival (avoid predation, find/attract a mate, locate sustenance). The auditory pathway in birds and mammals reflects the complexity of sound localization in the multitude, organization and anatomy of nuclei and neurons required to perform this task and additionally serves as a model system for other neural circuits which may be less organized or serve unknown functions. This review will provide a general overview of acoustical processing, starting in the cochlea and ending with the ITD machinery found in the superior olivary complex (SOC). To frame this discussion of the neural circuitry involved in sound localization, I will review two classic studies, Lord Rayleigh's duplex theory of sound localization and Lloyd Jeffress's coincidence detection model.

Modern auditory science began when Lord Rayleigh (John Strutt) published his psychophysical studies on the perception of sound frequency and intensity. Rayleigh reasoned that sound is localized in two ways, depending on frequency. In several simple experiments using tuning forks (to produce low frequency sounds), gas bags (to produce high frequency sounds) and observational analysis, Rayleigh developed the duplex theory of sound localization (Strutt, 1876, 1877). Rayleigh found that when a high frequency sound strikes a person's head from the side, the perceived intensity of the sound is reduced by blocking the ear closest to source, but if one blocks the opposite ear (furthest from the sound) the intensity is not affected (Fig. 2-1). Hence, an acoustic shadow is cast, resulting in a sound being louder on the exposed side relative to the side in the shadow (Strutt, 1877). This phenomenon is called interaural level difference, or ILD. Per contra, Rayleigh observed that when a subject was exposed to low frequency sounds, blocking either ear had no appreciable affect on sound intensity between the 2 ears; thus, he proposed that low frequency sounds wrap around the subject's head, reducing ILDs to indistinguishable differences (Fig. 2-1). Rayleigh hypothesized that a subject employs the time delay created when sound strikes one ear before the other (interaural temporal delay, ITD) to localize low frequency sound. Several decades later, Lloyd Jeffress proposed a coincidence detection model describing a circuit that could encode ITDs (Jeffress, 1948). According to the Jeffress model, two subthreshold inputs, one from each ear, travel along neural delay lines that terminate on an array of neurons in the brain. These neurons, which are tuned to different ITDs via differences in the 
lengths of their delay lines, only generate action potentials when their inputs arrive close enough in time to sum to supra-threshold levels. Figure 2-2 is a diagram of a coincidence detection circuit on the right side of the brain. If a sound, for example, arrives from the right then the input would reach the right ear and thus point $X$ before reaching the left ear and point $Y$. The signal would travel down the blue delay lines crossing nodes $1-3$ and reaching node 4 when the signal from the left is entering the delay line structure near point $Y$. Both signals arrive in coincidence only at node 5 to activate the tertiary fiber. A stimulus arriving to the left ear before the right mirrors the previous description, the signal reaches nodes $5-2$ unilaterally before bilateral coincidence occurs at node 1 . The location of this nucleus that coded for ITDs was, at that time, unknown; however, morphological evidence pointed to an area in the brainstem known as the superior olivary complex (SOC), and more specifically, the medial superior olive (MSO) (Stotler, 1953). Sensitivity to the location of low frequency sounds in the SOC is governed by small differences in the arrival time of excitatory and inhibitory inputs to the MSO. Below is a description of the anatomy and physiology of the auditory system in relation to the coding of low frequency sounds.

\section{B. Auditory Nerve}

Spiral ganglion neurons extend dendrites to hair cells in the snail shaped cochlea and project axons, which constitute the auditory nerve, to the cochlear nucleus (CN) (Lorente de No, 1981). Transduction of sounds into electrical signals occurs in the cochlea, which is tonotopically organized such that the base, nearest the round window, is tuned to high frequency sounds, and the apex is tuned to low frequency sounds. The audible spectrum varies from species to species. In humans the frequency range is from $20 \mathrm{~Hz}$ to $20 \mathrm{kHz}$ while in other species frequency response varies from a low frequency limit of $17 \mathrm{~Hz}$ to $12 \mathrm{kHz}$ in elephants (Heffner and Heffner, 1982), and a high frequency limit over $110 \mathrm{kHz}$ in echolocating bats (Koay et al., 2002). Transduction of sound from a pressure wave to an electrical signal is accomplished by displacement of stereocilia in response to resonating vibration of the basilar membrane and organ of Corti. Deflection of stereocilia activates mechanically coupled ion channels resulting in the depolarization of the hair cell at its base and the release of the neurotransmitter glutamate onto auditory nerve afferents. Spiral ganglion neurons have spontaneous rates or resting discharge rates ranging from $0 \mathrm{sp} / \mathrm{s}$ to well over $100 \mathrm{sp} / \mathrm{s}$. 
Recordings from their axons, referred to as auditory nerve fibers (ANFs), reveals three populations based on spontaneous discharge rate and threshold in cats. High-spontaneous rate units, defined as those cells having discharge rates over $18 \mathrm{sp} / \mathrm{s}$, had low thresholds. Fibers having discharge rates below $0.5 \mathrm{sp} / \mathrm{s}$ and a range of thresholds from 10 to $60 \mathrm{~dB}$ were categorized as low-spontaneous rate units. Medium-spontaneous units had discharge rates which varied from 0.5 to $18 \mathrm{sp} / \mathrm{s}$ and low thresholds typically less than $20 \mathrm{~dB}$ (Liberman, 1978). Action potential trains generated in auditory nerve fibers demonstrate two discharge patterns, depending on the frequency of the stimulus. These discharge patterns are shaped by the ability of hair cell receptor potentials to track the vibrating basilar membrane and the ability of auditory nerve fibers to fire in response to depolarization of the hair cells. Low frequency sounds ( $<4 \mathrm{kHz}$ in cats, but at different cutoff frequency depending on species), which resonate in the cochlear apex, cause sinusoidal depolarization and hyperpolarization of hair cells, resulting in cochlear afferents phase-locking (Fig. 2-3, top) or discharging at approximately the same phase of each cycle of a periodic sound (Rose et al., 1967; Johnson, 1980; Weiss and Rose, 1988). Above $4 \mathrm{kHz}$ the cellular membrane, which acts as a low pass filter, cannot follow cycle-by-cycle bending of stereocilia and the hair cells instead respond with a steady depolarization. For these high frequency tones the auditory nerve fibers demonstrate a "Primary" discharge pattern (an initial well-timed action potential followed by a decrease in discharge rate eventually adapting to a rate greater than spontaneous levels, Fig. $2-3$, bottom). When a low frequency sound is very intense a larger portion of the basilar membrane oscillates, so that many hair cells at higher frequency locations depolarize in a rhythmic fashion, giving rise to a phase-locked response in cells that also demonstrate a primary discharge pattern to higher frequency sound. Auditory nerve fibers then project tonotopically to various areas of the CN (Lorente de No, 1981; Fekete et al., 1984; Rouiller and Ryugo, 1984; Rhode and Smith, 1985; Liberman et al., 1990). Discharge patterns are displayed as peri-stimulus time histograms (PSTH), which are plots of the AP firing probability over time before, during and following an acoustic stimulus.

More recently Dreyer and Delgutte (2006) have examined phase-locking ability of high frequency ANFs by utilizing more temporally complex stimuli such as SAM tones (Joris and Yin, 1992) and transposed tones, previously used to study neurons of the inferior colliculus (IC) (Griffin, 2005; Dreyer and Delgutte, 2006) and auditory perception (Bernstein and 
Trahiotis, 2002). A mathematical description of SAM tones can be found in the methods section. SAM tones, briefly, are pure tones whose amplitude and thus temporal envelope has been modulated sinuisoidally at frequencies that range from a few $\mathrm{Hz}$ to many $\mathrm{kHz}$ (Glattke, 1969; Frisina et al., 1985; Langner and Schreiner, 1988; Kim et al., 1990). Phase locking to pure tones is the result of a neuron firing at the same phase of a periodic waveform. With transposed or SAM tones the unit phase locks to the envelope of the waveform. In the case of SAM tones the envelope is a sinusoid and in the case of transposed tones the envelope is a half-wave rectified sinusoid. The envelope of transposed tones generates deflections in the cochlea that more resembles the deflection generated by pure tones. These envelopes have been described as being similar to speech phonemes in the lower frequency ranges (3-20 $\mathrm{Hz}$ ) and, in the higher frequency ranges (>100 Hz), associated with pitch vocalizations. Responses to SAM and transposed tones are unlike responses to pure tones in that their vector strengths (VS), a measurement of phase locking precision, decrease quickly with increasing intensity, which in part is due to discharge rate saturation and the stimulus waveform occupying a larger and larger area of excitation in the tuning curves. Aim 2 will examine phase locking to SAM tones in cells of the MNTB in the context of ITD processing.

\section{Cochlear nucleus}

Cells in the $\mathrm{CN}$ receive input from the ANFs and have excitatory projections to the inferior colliculus (IC), lateral lemniscus (LL) and the various cell groups comprising the SOC. The $\mathrm{CN}$ can be divided into two broad areas based on structure, the dorsal cochlear nucleus (DCN) and ventral cochlear nucleus (VCN) (Fig. 2-4). The DCN is a complex structure having several layers and an architecture that resembles the cerebellum (Mugnaini et al., 1980; Mugnaini, 1985). Physiological resemblance to the cerebellum is evident in synaptic long term depression found in responses of principal fusiform cells and cartwheel cells (Tzounopoulos et al., 2004). The main function of the DCN is to process complex sounds directionally filtered by the pinna and project this information to the central nucleus of the IC. The VCN, which can be subdivided into anterior and posterior regions (AVCN and PVCN), functions in part as a preprocessor, temporally refining and organizing input from the cochlea before projecting conditioned auditory representations to the sound localization machinery of the SOC. Because the VCN projection to the SOC drive and constrained response properties 
of the MSO, MNTB and LNTB neurons, the following text will dwell mainly on studies of the VCN, especially the AVCN and rostral PVCN. Studies of the CN have taken two different approaches to subdividing the $\mathrm{CN}$ into functional groups: (1) a physiological approach (Pfeiffer, 1966; Godfrey et al., 1975), and (2) an anatomical approach based on the distribution of cell types (Osen, 1969; Brawer et al., 1974) or the distribution of nerve fiber terminals (Lorente de No, 1933; Brawer and Morest, 1975). Two types of bushy cells, located in the AVCN and rostral PVCN, (Brawer et al., 1974) play important roles in sound localization. Spherical bushy cells (SBCs) and globular bushy cells (GBCs) demonstrate similar response properties and have projections terminating in the medial and lateral superior olives and the MNTB/LNTB, respectively. In the next section a historical review of the anatomy and physiology of the cochlear nucleus is presented.

\section{i. Cochlear Nucleus Anatomy}

As mentioned above, the $\mathrm{CN}$ consists of three sub-nuclei (Fig. 2-4). Anatomical experiments carried out in the 1960s identified nine cell types in the CN in cats, which established the terminology and organization applied to other species. The cell types were classified by physical appearance in the light microscope using Nissl and Glees staining (Osen, 1969). Octopus cells are restricted to the caudal part of the PVCN. Large spherical cells and small spherical cells are restricted to the rostral third of the AVCN and middle third of the AVCN, respectively. Multipolar cells are distributed throughout the VCN. Globular cells are located in the central region of the VCN, within and just caudal to the cochlear nerve root. In the DCN, pyramidal cells are restricted to the granular cell layer. Giant cells are found throughout the DCN. "Small" cells are found throughout the CN. Granular cells form a thin layer covering the entire $\mathrm{CN}$ and lamella separating the ventral and dorsal nuclei.

Goldberg and Brownell (1973) characterized responses of neurons in the AVCN located in the rostral pole, known as the large-celled zone (LCZ), and the more caudally located small-celled zone (SCZ) (Osen, 1969). Tonotopically organized from high to low (dorsocaudal to rostroventral), the characteristic frequency (CF) of cells located in the SCZ and LCZ ranged from $0.25 \mathrm{kHz}$ to $27 \mathrm{kHz}$ (CF was restricted by the upper limit of their sound system) and $0.25 \mathrm{kHz}$ to $10 \mathrm{kHz}$, respectively. Neurons in the SCR had tuning curve shapes that were dependent upon the cell's best frequency. Tuning curves are created by plotting 
threshold intensity at a range of frequencies. Cells with low CFs $(<3 \mathrm{kHz})$ had broad responses and the tuning curves had long tails extending to lower frequencies. Cells with high CFs had narrow tuning curves. Goldberg and Brownell quantified the shape of these curves by calculating a $Q_{10}$ factor and the slopes of the tuning curve at lower and upper frequency range. $Q_{10}$ is calculated by taking the ratio of $C F$ to width of the tuning curve $10 \mathrm{db}$ above threshold. Frequency dependence of $Q_{10}$ and slope steepness confirmed the dependency of tuning curve shape on CF. Most SCR cells had primary-like (PL) discharge patterns (similar to auditory nerve fiber responses) in response to tone bursts and those with CFs below $3 \mathrm{kHz}$ tended to phase-lock. Increasing the stimulus intensity led to a decrease in first spike latency, jitter and an increase in discharge rate. Two other measurements Goldberg and Brownell used to quantify a cell's discharge pattern were the coefficient of variation and vector strength. Coefficient of variation (CV), which provides information as to the regularity at which a cell discharges, is determined by calculating the ratio of standard deviation of intervals to the mean interval. SCR cells had irregular discharge patterns and thus high CVs. Vector strength, a measure of phase-locking precision to the stimulus period, was high in units that responded to low frequency sounds. The region referred to as the SCR by Goldberg and Brownell correlates to the AVCN. Although the response characteristics of ANFs and SCR cells are similar, a few differences exist. These differences, which can be attributed to the distribution of synaptic input and synaptic morphology, are demonstrated in better phase-locking (Joris et al., 1994b; Joris et al., 1994a) and the presence of PL with notch (PLN) PSTH patterns (Pfeiffer, 1966; Goldberg and Brownell, 1973).

In early studies, large and small nerve terminals were described in the AVCN, ranging from large end-bulbs of Held to small boutons (Cajal, 1909; Lorente de No, 1933). Cajal found that end-bulbs of Held became larger from the posterior AVCN to the anterior AVCN; however, these end-bulbs could not be unequivocally associated with a particular cell morphology. Further clarification of the relationship between synaptic geometry and distribution to cell morphology was established (Brawer and Morest, 1975) utilizing the Golgi method in cats. Brawer and Morest found no nerve terminals on the cell bodies of granule or small cells and no end-bulbs of Held associated with stellate cells. The end-bulbs of Held could only be seen in contact with the perikerya of spherical cells, which were also termed bushy cells based on dendritic arbor morphology. More importantly, a size discrepancy in 
end-bulbs existed among the bushy cells indicating possibly more than one type of bushy cell, in agreement with Cajal's study. Brawer and Morest speculated that the larger end-bulbs of Held could be responsible for the complex wave-forms (pre, then post synaptic potentials) found in recordings made from bushy cells of the AVCN (Goldberg and Brownell, 1973; Bourk, 1976). The prepotential, the first peak of a multi-peaked waveform, results when many large boutons depolarize synchronously or when a single large bouton depolarizes.

Prepotentials are recorded from cell types having large terminals on the cell body such as MNTB cells and bushy cells of the AVCN. The smaller end-bulbs require synchronicity to depolarize a cell and are incapable of generating visually detectable prepotentials, explaining the simpler waveforms recorded in the posterior AVCN. Prepotentials could be observed in these cells when voltage waveforms were averaged across multiple action potentials (AP)(Bourk, 1976). The soma of a GBC in cat receives anywhere from 9-69 (average 23) specialized synapses (modified endbulbs of Held) from the auditory nerve fiber (Spirou et al., 2005). At low frequency, these inputs are phase locked to the frequency of the sound and convergence imparts greater precision in phase locking to the GBC than is found in ANFs (Rothman et al., 1993; Smith et al., 1998; Spirou et al., 2005). An anatomical study by Cant and Morest in 1979 provided evidence that spherical cells defined by Nissl stain corresponded to bushy cells defined by Golgi stain. Nissl and Golgi stains performed in the $A V C N$ of cats showed correspondence in the locations of these cell types (Cant and Morest, 1979; Tolbert et al., 1982). Spherical and bushy cells were rounder and had similar diameters $(30.2 \pm 0.1 \mathrm{~mm}$ and $28.9 \pm 0.4 \mathrm{~mm})$ compared to stellate and small cells. Ergo, evidence existed at that time that many cells in the AVCN have PL responses to short tone bursts and that bushy/spherical cells likely comprise the cell types giving rise to that response.

\section{ii. Cochlear Nucleus Physiology}

$\mathrm{CN}$ cells exhibit a variety of response patterns to sounds. Russell Pfeiffer developed a classification scheme for PSTH response patterns using short tone bursts ( $25 \mathrm{msec}$ ) at each cell's CF, with the intensity delivered varying from threshold (visual detection level VDL) to 20 $\mathrm{dB}$ above threshold. The characteristic frequency (CF) is the frequency to which a cell is most sensitive, resulting in an increase of discharge rate above spontaneous levels. These response patterns are characterized as primary-like $(\mathrm{PL}), \mathrm{PL}$ with notch $\left(\mathrm{PL}_{\mathrm{N}}\right)$, Chopper, 
Pause, On and Buildup (Pfeiffer, 1966). The $P L$ and $P L_{N}$ patterns have a similar response pattern to that of the primary discharge pattern of auditory nerve fibers; however $\mathrm{PL}_{N}$ units have a notch $(0.5-2 \mathrm{~ms})$ in their discharge pattern when no spikes occur after the initial welltimed spike. Chopper cells have regularly spaced periods of discharge that for some cells become more distinct at higher sound pressures. Other units having chopper responses at low stimulus intensities demonstrate pause discharge patterns at higher stimulus intensities (Pfeiffer, 1966). Chopper responses can be further characterized as sustained choppers (Godfrey et al., 1975) or transient choppers (Bourk, 1976) using discharge regularity throughout the stimulus length, as measured by CV (Young et al., 1988). Cells defined as Pause units respond initially with 1 or 2 action potentials followed by $5-100 \mathrm{~ms}$ of silence and then an increase in discharge rate, and On units respond with an initial one or two spikes followed by discharge rate that is $0 \mathrm{sp} / \mathrm{s}$ or close to the spontaneous discharge rate. The last category of response patterns, called buildup units, resemble pause units but lack the initial one or two spikes. Pfeiffer did not associate these response patterns with any particular cells; however, they were localized to several sub-nuclei of the CN. PL and Pause response patterns were found exclusively in the VCN and DCN respectively. Chopper and On responses are associated with the PVCN; however, these response types can be found throughout the $\mathrm{CN}$. In 1976 Bourk proposed that $\mathrm{PL}$ and $\mathrm{PL}_{\mathrm{N}}$ discharge patterns correspond to spherical bushy cells (SBCs) and globular bushy cells (GBCs) respectively of the AVCN in Nissl stained tissue. A series of studies in cats, using horseradish peroxidase to intracellularly label physiologically characterized neurons, cemented firm associations between response types and cell morphologies (Rhode et al., 1983; Rouiller and Ryugo, 1984). Stellate cells demonstrate chopper discharge patterns in response to pure tones (Rhode et al., 1983; Rouiller and Ryugo, 1984). Onset responses are associated with two morphologies, octopus cells in the octopus cell area of the PVCN and stellate cells found in the multipolar area (Rhode et al., 1983). Spherical bushy cells found in the AVCN were correlated with PL discharge patterns and GBCs located in the nerve root area responded to tone stimuli with $\mathrm{PL}_{\mathrm{N}}$ (Smith and Rhode , 1985), On-L and PL discharge patterns (Rhode, 2008). A similar study in the rat VCN utilized intra-axonal injections of horseradish peroxidase to make similar associations between morphological characteristics and PSTH response types (Friauf and Ostwald, 1988). 
Another classification scheme, which we did not utilize but is used in characterizing the responses of auditory units examines the frequency response map. Frequency response maps are similar to tuning curves except the discharge rate is plotted at several different intensities across frequency and have the advantage of revealing the characteristics of inhibitory inputs. Units are classified as types I through $\mathrm{V}$ depending on response map shape, which is dependent on the interplay of excitatory and inhibitory inputs (Evans and Nelson, 1973; Young and Brownell, 1976). Therefore, many of these studies have been carried out in the decerebrate cat. Type I units respond to noise and tones but have no inhibitory side bands sharpening the tuning in their response maps. Type II units are activated by tones but show little or no activation when stimulated with noise and are not associated with any distinct response pattern to short tone bursts. Type III units are similar to type I units but have inhibitory side bands. The type IV response map is complex; cells respond to noise and CF tones at threshold but are inhibited across a wide frequency range and at higher stimuli intensities at CF. Generally these cells have two excitatory regions and two inhibitory regions. The excitatory regions, which can be connected, are found in a small area around CF and are limited to intensities around threshold and a narrow region at higher frequencies and greater intensities. The two inhibitory regions found in type IV are found flanking the high frequency excitatory region and at CF at higher sound intensity. There is also a broad region below CF that can be excitatory or inhibitory (Young and Brownell, 1976; Spirou and Young, 1991). Type $\mathrm{V}$ responses are purely inhibitory with discharge rates falling below the spontaneous discharge rate at CF (Evans and Nelson, 1973). In a comprehensive examination of two categorization schemes in decerebrate cats, PSTH type and receptive field (response maps), Shofner and Young found that the two schemes were not independent (1985). Types II through IV were found in the DCN. Units in the VCN had primarily PL, $P L_{N}$ and chopper PSTHs and were classified as types I and III, and cells localized to the AVCN demonstrated exclusively type I responses.

Additional physiological distinctions can be made between a cell's inputs and its resulting output by exploring a unit's response properties at non-CF frequencies or by utilizing more complex stimuli. In the AVCN, stimuli have been restricted to sounds that would help determine levels of phase locking ability due to their importance in low frequency sound localization. Phase locking to pure tones can be measured in cells with CFs less than $4 \mathrm{kHz}$ 
(Rose et al., 1967; Johnson, 1980) or in cells with higher CFs by exciting them with low frequency tones that reside in the tail of their tuning curve (Joris et al., 1994a). A subpopulation of globular and spherical bushy cells were found to have higher synchronization coefficients (symbolized as ' $R$ ') than auditory nerve fibers when stimulated with a $500 \mathrm{~Hz}$ pure tone. Though not as robust as trapezoid body fibers, high frequency ANFs had high $R$ values compared to low frequency ANFs when stimulated with the $500 \mathrm{~Hz}$ tone. Additionally, the phase locking ability of AVCN cells tuned to higher frequencies can be measured by presenting SAM tones or transposed tones (in brief a half-wave rectified SAM tone). Responses of AVCN to transposed tones have not been studied, but responses to SAM tones have been used to characterize PL and $\mathrm{PL}_{N}$ cells in the VCN (Rhode and Greenberg, 1994). Temporal modulation transfer function (tMTFs) can be generated by plotting the synchronization coefficient versus the modulation frequency of the SAM tone; these plots generally take the shape of a low pass filter (phase locking deteriorating as modulation frequencies increase) or band pass filter (units having a preferred modulation frequency). Rhode and Greenberg found that $P L$ and $P L_{N}$ units have tMTFs that resemble tMTFs of ANFs, except that they tend to be slightly band pass shaped. The mean peak synchronization values were higher for $\mathrm{PL}_{N}$ than $\mathrm{PL}$ units $(0.78$ vs 0.56$)$. These units also had corner and cutoff frequencies of $0.5 \mathrm{kHz}$ and $1.3 \mathrm{kHz}$ respectively, which were similar to ANFs. Cut off frequency in this experiment was defined as a synchronization coefficient of less than 0.1 and the corner frequency was defined as the point at which the synchronization coefficient drops below $80 \%$ of the maximum synchronization coefficient. Because natural sounds do not consist of long pure tones but rather sounds that sweep across frequency and vary temporally, SAM and transposed tones provide a method of investigating the processing of natural sounds and species-specific communication in the SOC. Additionally SAM tones provide an easily generated stimulus with known characteristic that we utilized to investigate the phase-locking ability of high frequency cells in the MNTB (Aim 2).

In summary, two types of bushy cells (Brawer et al., 1974) play important roles in low frequency sound localization. SBCs and GBCs of the AVCN (Cant and Morest, 1979) behave similarly in response to pure tones with $\mathrm{PL}$ and $\mathrm{PL}_{N}$ discharge patterns and both project to cell groups involved in low frequency sound localization (Cant and Casseday, 1986). GBCs receive several specialized inputs called modified endbulbs of Held and these bushy cells 
project mainly to the contralateral MNTB with a collateral branch in the ipsilateral LNTB (Tolbert et al., 1982; Spirou et al., 1990; Liberman, 1991; Smith et al., 1991). SBCs receive input in the form of several large synapses called end-bulbs of Held and have projections terminating in the LSO and MSO. These terminal projections in the MNTB, LNTB and MSO shape and constrain ITD coding in mammals.

\section{Superior Olivary Complex}

In the brainstem of all mammals lies the anatomically diverse SOC, where complex binaural processing of acoustic stimuli occurs. The size and number of nuclei vary across species, and are determined and constrained by head size and the animal's acoustic environment. Different cell groups of the SOC receive inputs from different cells of the $\mathrm{CN}$ and perform different roles in sound processing and localization. The three most prominent nuclei of the SOC, the lateral superior olive (LSO), medial superior olive (MSO) and medial nucleus of the trapezoid body (MNTB), are conserved across species and receive inputs from the $\mathrm{CN}$, neighboring nuclei in the $\mathrm{SOC}$, as well as descending projections from the lateral lemniscus, IC and higher auditory structures.

The duplex theory of sound localization predicts that because high frequency sounds and low frequency sounds are processed differently, the neural circuitry encoding ILDs (of high frequency sounds) and ITDs (of low frequency sounds) would be separate. The IC was shown to contain cells having low CFs that were sensitive to temporal differences in the onset of binaural tones. Other cells in the IC were sensitive to small changes in the intensity of binaural sounds: discharge rates decreasing as the intensity of the ipsilateral sound increased (Rose et al. 1966). Galambos et al. found cells of the accessory nucleus (i.e. MSO) to be sensitive to time differences in the onsets of binaural clicks (1959). Whether ITD and ILD processing starts in the IC or in nuclei of the SOC projecting to the IC was unknown at that time; however, later studies confirmed that MSO neurons are ITD sensitive (Goldberg and Brown, 1969; Yin and Chan, 1990; Spitzer and Semple, 1995), and LSO neurons are ILD sensitive (Boudreau and Tsuchitani, 1968, 1970; Caird and Klinke, 1983).

MSO neurons receive bilateral excitatory input from the $\mathrm{CN}$ and inhibitory input from the contralaterally driven MNTB and ipsilaterally driven lateral nucleus of the trapezoid body (LNTB) (Kiss and Majorossy, 1983; Spangler et al., 1985; Cant and Hyson, 1992; Kuwabara 
and Zook, 1992). Because recent studies have shown that inhibition modulates ITD sensitivity in the MSO (Brand et al., 2002; Pecka et al., 2008a), a deeper understanding of how these inhibitory cell groups respond to sound is warranted.

\section{i. MNTB}

Cells of the MNTB receive input from globular bushy cells (GBC) of the contralateral cochlear nucleus in the form of a single large nerve terminal, the calyx of Held, enveloping the soma (Cajal, 1909; Morest, 1968; Friauf and Ostwald, 1988; Spirou et al., 1990; Smith et al., 1991). The primary target of MNTB neurons is the LSO (Glendenning et al., 1985; Spangler et al., 1985), although collaterals of many MNTB cells terminate in the MSO (Spangler et al., 1985; Kuwabara and Zook, 1992), the lateral lemniscus (Glendenning et al., 1981), the MNTB itself (Smith et al., 1998) and other periolivary regions (Spangler et al., 1985; Smith et al., 1998). To understand the functional effect of inhibition in an ITD circuit, it is necessary to understand the physiological response of MNTB neurons to low frequency stimuli and stimuli rich in temporal features.

Due to the large size and encompassing nature of the calyx of Held, the MNTB has been thought to act as a signal inverter, turning precisely timed excitation into precisely timed inhibition. The calyx of Held is a large enough current sink that it generates a prepotential that is visible without signal averaging in extracellular recordings made in MNTB cells of cats (Guinan and $\mathrm{Li}, 1990$ ). However, the prepotential and postsynaptic potential are not always one-to-one, indicating failure of excitatory synaptic transmission or inhibition prevailing from non-calyceal inputs (Kopp-Scheinpflug et al., 2003b; Kopp-Scheinpflug et al., 2008). More recent studies in cats showed that calyceal failures are infrequent. Estimates of higher failure rates may actually be recordings from nearby calyces and the result of the close proximity of cells in the MNTB. Anatomical data suggest that, regardless of the synaptic security of the calyx of Held to the MNTB, other excitatory and inhibitory terminals modulate its discharge rate. Electron micrographs reveal that $25-50 \%$ of the soma is covered in terminals having round excitatory vesicles originating from small boutons and from the calyx of Held. Roughly $20 \%$ of the terminals have flattened vesicles, indicating inhibitory synapses (Smith et al., 1998). Supporting these EM studies, immunocytochemical labeling reveals the presence of 
GABAergic and glycinergic boutons on the cell bodies (Roberts and Ribak, 1987; Adams and Mugnaini, 1990; Spirou and Berrebi, 1997).

One to two dendrites extend from the cell body, giving rise to a complex, profusely branching dendritic tree. The proximal dendrites are innervated similarly to the soma, having approximately $65 \%$ of the surface covered in boutons, half from round vesicles. While these sparsely innervated dendrites might only be capable of driving the cell in conjunction with non-calyceal inputs found on the cell body (Hamann et al., 2003), $\mathrm{Na}^{+}$channels located on the dendrites maintain AP dynamics which contribute to the ability of MNTB neurons to discharge at high rates (Leão et al., 2008). Inhibitory inputs have been proposed as originating from nearby MNTB cells (Kuwabara and Zook, 1991; Smith et al., 1998), the ventral nucleus of the trapezoid body (Ostapoff et al., 1997), and possibly other SOC nuclei. The effect that these inhibitory inputs have on MNTB cells is not clear because most of these studies were conducted in anesthetized animals.

An aside, we performed our studies in an unanesthetized decerebrate preparation in contrast to the majority of studies in the SOC, which have been performed in anesthetized animals and thus under the effects of synaptic modulation from these drugs. Typical usage of pentobarbital and ketamine as anesthetics have been shown to affect GABAergic inhibitory inputs (Richter and Holtman, 1982) and NMDA excitatory inputs (Anis et al., 1983) respectively. Responses gathered in the absence of or with reduced anesthetic doses in many species have shown faster responses, higher spontaneous discharge rates as well as more complex stimuli (Evans and Nelson, 1973; Young and Brownell, 1976; Batra et al., 1989, personal communication Felix, R.). Decerebration, that is transection of the superior colliculus (SC) just rostral of the inferior colliculus, preserves local inhibitory circuitry in the SOC and within the entire auditory brainstem caudal of the SC.

MNTB cells have response characteristics much like their primary input, and their output reflects the precision and response types of GBCs. In cells with low CF, action potentials will be highly phase-locked $(R \geq 0.9)$ to the phase of the stimulus; however, in high CF cells, the PSTH response will be PL or $\mathrm{PL}_{\mathrm{N}}$ (Joris et al., 1994b). For a GBC with a CF between $1-3 \mathrm{kHz}$, a tightly phase-locked response could be elicited by driving the cell with a tone less than $1 \mathrm{kHz}$ (Joris et al., 1994a). This behavior has not been tested for MNTB cells, although one can assume that a similar response would be found. Another study in rat MNTB 
revealed neurons with CFs ranging from $14 \mathrm{kHz}$ to $47 \mathrm{kHz}$. No low frequency $(<14 \mathrm{kHz})$ cells were found in this study, most likely due to the under representation of low frequency cells in the rat auditory system, or possibly due to a limitation in the piezoelectric speakers used for the experiment whose maximum sensitivity was between 10-55 kHz (Sommer et al., 1993). A more recent study examining tone duration on discharge rate found MNTB cells in rat tuned to a broader range of frequencies, from 1.5 to $56 \mathrm{kHz}$ (Kadner et al., 2006).

To examine the influence of inhibition in the MNTB, Kopp-Scheinpflug et al. (2003a) used a two-tone stimulus consisting of a probe tone at the CF of the cell lasting $100 \mathrm{~ms}$, and a test tone (not at characteristic frequency) which was delayed $30 \mathrm{~ms}$ to the probe tone and lasting only $40 \mathrm{~ms}$. These cells exhibited a decreased firing rate during the test tone stimulation without a decrease in calyceal prepotential discharge rate (Kopp-Scheinpflug et al., 2003b). Furthermore, it has been shown in rats (in vitro) that glycinergic inhibition can shunt action potentials generated in MNTB cells when the inhibitory post synaptic potentials (IPSPs) precede the calyceal driven EPSPs (Awatramani et al., 2004). Strong evidence exists for inhibitory inputs modifying MNTB output; however, the transformation, which these inhibitory projections modify, must be characterized better.

Aim two addresses several issues: (1) Few recordings have been made in the lowfrequency lamina of the MNTB and therefore these cells are not well characterized. This issue is important because low CF MNTB cells likely provide the contralateral phase locked inhibition that MSO cells utilize in ITD processing. (2) Response latencies to clicks and low frequency tones have not been determined. These data can help determine whether inhibition can precede excitation in MSO cells. (3) It is currently unknown, in the unanesthetized cat, if all MNTB principal cells reflect the firing pattern of their calyceal input by exhibiting similar PSTH patterns.

\section{ii. $L N T B$}

Located laterally to the MNTB and MSO, the LNTB is one of the largest periolivary cell groups (PON) of the cat SOC and has projections terminating locally in the SOC as well as the CN (Elverland, 1978; Kuwabara and Zook, 1992). Due to the complex geometry of PON and a multitude of cell types, delineation of cell groups proves difficult. Indeed, some debate existed as to whether the pale oval cells found capping the caudal end of the LNTB and 
extending rostrally and parallel to the LNTB was a unique nucleus, termed the posteroventral periolivary nucleus (pvPO, Warr, 1969; Tsuchitani, 1977), or whether it was a sub-nucleus of the LNTB. A series of anatomical studies by Spirou and Berrebi (described below) addressed these issues by categorizing and associating the terminal patterns of LNTB cells, visualized with PEP-19, with the Nissl appearance.

The LNTB was subdivided into three nuclei by Spirou and Berrebi (1996, 1997), based on cell morphology, including ultrastructure, immunoreactivity to glycine and GABA and the types of nerve terminals synapsing on these cells, visualized in part using immunolabeling for PEP-19, a putative $\mathrm{Ca}^{2+}$ binding protein. Three distinct patterns of labeled terminals and fiber size are associated with the mLNTB, pvLNTB, and hLNTB and are correlated with morphology of these cell bodies in Nissl stained tissue. Pattern 1: mLNTB is characterized by variable fiber size and a high density of differently sized puncta surrounding the cells. Cell bodies are multipolar in shape and have clumps of Nissl substance in the soma. Pattern 2: pvLNTB is defined by large PEP-19 immunoreactive (IR) axons and large puncta, often connected via small PEP-19-IR labeled branches surrounding immuno-negative cells. These observations correlated with the pale oval cells in Nissl stained tissue. Pattern 3: hLNTB found in the ventral hilus of the LSO had few thin immunolabeled fibers and small puncta. Terminals surrounding hLNTB cells often were weakly PEP-19 immuno-reactive and few cells were immunoreactive. The large terminals contacting pvLNTB neurons are branches of GBCs, as revealed by retrograde and intracellular labeling (Tolbert and Morest 1982; Smith et al. 1991). Thus, we hypothesize that MNTB cells and pvLNTB cells integrate inputs similarly. Therefore, pvLNTB cells likely have low first spike response latencies and respond to tone stimuli with PL and/or phase locked discharge patterns, similar to MNTB cells.

Inhibitory projections from the LNTB terminating in the CN (Spangler et al., 1987) and MSO (Cant and Hyson, 1992) were studied prior to the classification by Spirou and Berrebi, and so did not take into account their parcellation of the LNTB. Therefore, which sub-nuclei project to MSO and play important roles in ITD coding is unknown. The largest subnuclei, mLNTB and pvLNTB, could each provide significant innervation of the MSO. The MNTB projects glycinergic terminals to the MSO; however, immunohistochemical studies of the MSO show terminals that are both $\gamma$-aminobutyric acid (GABA) and glycine immunoreactive (see MSO section). Additional evidence in the similarities between pvLNTB cells and MNTB 
cells include strong glycine-IR of pvLNTB cells which matches that of MNTB cells, and pvLNTB, like MNTB, is devoid of GABA-IR (Spirou and Berrebi, 1997). Comparatively, the $m L N T B$ is less immunoreactive for glycine; few cells showed the intensity of staining found in the pvLNTB, but most mLNTB cells showed some glycine-IR. Immunoreactivity to GABA was only found in cells that were also immunoreactive to glycine and only found in approximately $44 \%$ of glycine-IR cells (Spirou and Berrebi, 1997).

Physiological studies preceded the majority of anatomical investigations of the LNTB, and as a result these studies do not distinguish the location of response characteristics among sub-nuclei of the LNTB. One such study found 24 ipsilaterally driven units, located in the LNTB, that were organized tonotopically from high $(11 \mathrm{kHz})$ to low $(200 \mathrm{~Hz}) \mathrm{CFs}$, ventromedial to dorsolateral (Tsuchitani, 1977) in accordance with the tonotopic map of the LNTB established by Guinan et al (1972a). Response latencies for these cells ranged from 5$8 \mathrm{~ms}$ for a pure tone having a $1 \mathrm{~ms}$ onset ramp. LNTB cell responses to pure tones were characterized as chopper and PL; however the sample size was small so that no association could be made between response type and CF. Chopper type responses are associated with cells located in rostral LNTB, which Spirou and Berrebi have shown to have few pvLNTB neurons. mLNTB units may provide phasic inhibition to MSO at low stimulus frequency, as measured from VCN chopper cells, and tonic inhibition at higher frequency, also similar to VCN chopper responses (Blackburn and Sachs, 1989; Wiegrebe and Meddis, 2004).

In summary, pvLNTB, like MNTB, receives input via large terminals, likely from VCN GBCs, and was proposed to provide MSO cells with phase locked inhibition similar to MNTB (Berrebi and Spirou, 1998). Input to the mLNTB may originate from VCN GBCs, SBCs and stellate cells (unpublished, Spirou an Berrebi). Given the variety in terminal size on mLNTB cells, patterns of GABA-IR, glycine-IR and co-localization in some cases, as well as chopper PSTH responses, these cells likely do not phase-lock as well as pvLNTB cells and instead provide tonic inhibition across stimulus frequency. Long duration, tonic inhibition has been shown in cellular models to improve ITD sensitivity of MSO and nucleus laminaris neurons (Reyes et al., 1996; Zhou et al., 2005). In aim 1 we examine the populations of these subnuclei through stereological counts and retrograde injections in the MSO. 


\section{iii. MSO}

Bipolar MSO cells receive excitatory bilateral projections from the cochlear nucleus and receive inhibitory inputs from the MNTB and subnuclei of the LNTB. The mechanism and the degree to which these inhibitory nuclei modify the output of MSO cells is still unknown and addressed in Aim 3. However, a population rate code has been proposed as an alternative to the Jeffress place code as the mechanism by which ITD is coded within the MSO. To facilitate the discussion of the currently available experimental evidence, included is a detailed overview of the anatomy (provided above and below) and existing model for the encoding of sound location using ITD.

\section{a. MSO Anatomy}

With the first modern investigations of neuroanatomy, Cajal and later Winkler utilized silver and ablation/degeneration techniques to visualize nuclei in the brainstem (Winkler, 1907; Cajal, 1909). Cajal described one of these nuclei, termed the accessory superior olivary nucleus, as a stack of cells with their long axes parallel to the horizontal plane, appearing semi-lunar when viewed coronally (Fig. 2-5). The accessory superior olivary nucleus was later renamed the medial superior olive (MSO) due to its prominence in primates, cats and dogs, and location relative to the main olivary nucleus, which was also renamed the lateral superior olive. When viewed coronally, the MSO is a stack of bipolar cells 3 to 4 cell bodies thick (Fig. 3-2), with dendrites extending outward more than 200 microns from the medial and lateral sides of each cell. The axons usually originate from one side of the cell body and sometimes from the dendrites (Smith, 1995).

Innervation of the MSO from the $\mathrm{CN}$ was initially characterized using classical degeneration techniques (Stotler, 1953). Stotler found that ablation of the $\mathrm{CN}$ resulted in degeneration of the neuropil on the proximal side of the ipsilateral MSO, and if animal survival times were long enough, degeneration of the neuropil on the proximal side of the contralateral MSO as well. By lesioning the IC, retrograde chromatolysis revealed that MSO axons project through the lateral lemniscus to the inferior colliculus unilaterally. Since Stotler's early experiments, many studies, such as those described below, have further defined the details of innervation, cell morphology, tonotopic organization and post-synaptic targets of the MSO. Afferent fibers from the $\mathrm{CN}$ reach the MSO by passing through the MNTB 
on the medial side, and passing under or penetrating the LSO on the lateral side (Morest, 1973; Perkins, 1973). EM studies found boutons of varying size containing round vesicles (excitatory) on the soma and dendrites of MSO cells. Boutons containing flattened vesicles (inhibitory) were restricted to the soma and proximal dendrites (Clark, 1969a). Lesions of the $\mathrm{CN}$ caused degeneration of nerve terminals that contained round synaptic vesicles, which were likely excitatory inputs (Perkins, 1973; Lindsey, 1975; Kiss and Majorossy, 1983; Russell and Moore, 1999). These lesions spared endings with pleomorphic or flattened vesicles, which were likely inhibitory inputs (Uchizono, 1965).

Although MSO cells in the cell column appear bipolar in coronal section, a range of dendritic projections have led some investigators to identify additional cell classes that are multipolar and include rostro-caudally projecting dendrites (Scheibel and Scheibel, 1974; Lindsey, 1975; Schwartz, 1977; Kiss and Majorossy, 1983). Spinous multipolar neurons, which receive few synapses and, based upon HRP injection, were described as projecting locally, are centrally located and have spherical dendritic arbors. Kiss and Majorossy proposed that these cells have a local inhibitory function. Elongated marginal cells, which are typically unilaterally innervated by the $\mathrm{CN}$, have cell bodies and dendrites that are restricted to the perimeter of the MSO; however one or two dendrites often extend parallel to fusiform dendrites with very few dendrites extending into other periolivary nuclei.

In the 1970s, Guinan described tonotopic organization of the MNTB and MSO in cat. Low CF cells are located in the dorsal MSO and cells having progressively higher CFs are located more ventrally (Guinan et al., 1972a). Tonotopy of MSO in other species has been described using anatomical and physiological techniques. Muller (1990) used anterograde tract tracing techniques to show that AVCN cells in gerbils with CFs lower than $3.5 \mathrm{kHz}$ projected to the MSO. Tonotopic organization extended throughout the rostral-caudal axis of the MSO resulting in a redundancy of frequency representation.

In birds, evidence exists for not only tonotopic organization, but Young and Rubel found a delay line structure in the excitatory projections from the nucleus magnocellularis (NM, AVCN homologue) to the nucleus laminaris (NL, MSO homologue)(Young and Rubel, 1983). These axonal delay lines form from the ipsilateral and contralateral NM innervating the NL on its dorsal and ventral surfaces, respectively (Carr and Konishi, 1988). Furthermore, intracellular recordings, in owls, showed that movement of the electrode dorsally to ventrally 
along an isofrequency lamina resulted in increased temporal delay for ipsilateral stimuli and decreased temporal delay for contralateral stimuli. This arrangement of afferent axons is consistent with a place code for ITD sensitivity, in effect supporting the Jeffress model.

While there is strong evidence for a place code constructed with delay lines in aves, the anatomical evidence for delay lines in mammals is more complicated. An early study found projections from the contralateral $\mathrm{CN}$ terminating in the rostral MSO before terminating in the caudal MSO; in contrast, projections from the ipsilateral CN showed no such organization (Smith et al., 1993). These results do not rule out a Jeffress type model, as only one set of delay lines is needed to establish locotopic tuning across a nucleus. More recent reconstructions of contralateral AVCN projections support caudally directed delay lines, but reveal more complex projections from the ipsilateral $A \bigvee C N$, demonstrating several distinct innervation patterns (Beckius et al., 1999; Karino et al., 2011). Some ipsilateral projections terminate in restricted delay lines that extend rostrally, while other terminal fields had collaterals having no discernable differences in axonal length (Beckius et al., 1999). Karino et al. found a third arrangement that mirrored the contralateral pattern of caudally directed delay lines in 3 of 7 ipsilateral reconstructions (2011). The consequence of this last innervation pattern would be that inputs to MSO cells would be of equal length, at least locally, and would not support a Jeffress place code. Several studies have attempted to predict conduction velocity of these SBC fibers (Beckius et al., 1999; Karino et al., 2011) by estimating axonal diameters. However, without accurate anatomical measures of diameter, and given the strong dependence of conduction velocity on distance between nodes of Ranvier (Seidl et al., 2010), which is typically not measurable, it remains difficult to obtain good estimates of conduction velocity.

Inhibitory projections to the MSO, briefly discussed in previous sections (MNTB and LNTB), undoubtedly add to the complexity of ITD processing and are addressed in aims 1 and 3 of this dissertation. The possibility of a set of inhibitory delay lines overlaying the excitatory delay lines is quite real and can only be investigated through reconstructions of labeled LNTB and MNTB cells. MNTB axons have collaterals terminating in the MSO (Smith et al., 1998; Kuwabara and Zook, 1992; Spangler et al., 1985) and through careful reconstruction of terminal fields Kuwabara and Zook showed that the MNTB and LNTB are the source of inhibitory inputs to MSO soma. MNTB to MSO connections were tonotopic 
(Kuwabara and Zook, 1992); however, collaterals could be seen innervating MSO cells rostral and caudal to the points where the fiber entered this nucleus (Smith et al., 1998; Kuwabara and Zook 1992). No such organization was apparent in these studies in regards to LNTBMSO connections and is one of the issues discussed in Chapter 3.

\section{b. MSO Physiology}

The earliest physiological evidence of ITD processing in the brainstem appeared in a study by Galambos et. al. in which cells of the accessory nucleus (MSO) were found to be sensitive to time differences in the onsets of binaural clicks (Galambos et al., 1959). Another early single cell recording study found that the IC contained cells sensitive to differences in the phase of binaural low frequency sounds (Rose et al., 1966).

The first substantial experimental evidence for how MSO cells code ITD came from Goldberg and Brown (1969). Of 15 neurons sensitive to ITDs, six were located in the MSO and the remaining cells were located dorsally in the medial periolivary nucleus (MPO). Goldberg and Brown found that MSO cells sensitive to monaural stimuli from either ear typically respond more strongly to one ear. For binaural stimuli, discharge rates of MSO neurons vary in a time/phase dependent manner such that at the most favorable delay the discharge rate is at least the sum of the monaural discharge rates. At the least favorable delay, cells have discharge rates less than either monaural discharge rate and sometimes lower than the spontaneous discharge rate. This observation could result from inhibition actively suppressing MSO cells from firing. Neurons having low CFs discharged in a phaselocked fashion, reflecting their phase-locked excitatory input from SBCs (Moushegian et al., 1975). Goldberg and Brown showed that maximum discharge rate is achieved when the phase-locked ipsilateral and contralateral inputs reach the cells in phase, and that the rate is minimized when the inputs are out of phase. In another comprehensive study of MSO firing characteristics, Yin and Chan showed that MSO neurons are coincidence detectors (Yin and Chan, 1990). In both of these studies, most MSO cells fired maximally when the ipsilateral input lagged the contralateral input (Goldberg and Brown, 1969; Yin and Chan, 1990), an indication that contralateral inputs have a longer transit time to the MSO than ipsilateral inputs. 
Many investigators have encountered difficulty in recording from the MSO, which is located deep within the brainstem (Caird and Klinke, 1983, Goldberg and Brown, 1969). In 1990, Yin and Chan provided a comprehensive overview of ITD sensitive MSO cells and evidence of a delay line organization of the MSO. Of the 39 cells localized to the MSO, only one had a characteristic frequency greater than $3 \mathrm{kHz}$, as data was biased towards low frequency units, and about $80 \%$ were sensitive to ITDs or interaural phase differences (IPD) of pure tones (Yin and Chan, 1990). Additionally, cells showed ITD sensitivity when exposed to broad-band stimuli in the form of a pseudo-gaussian noise limited to $60-4000 \mathrm{~Hz}$. Yin and Chan showed that the MSO was not only tonotopically organized in accordance with other studies (Guinan et al., 1972a; Muller, 1990; Oliver et al., 2003), but also spatially organized such that cells located in the anterior pole of the MSO had peak ITDs near zero, and peak ITDs shifting to positive delays (contralaterally leading stimuli) in the posterior region. Due to the paucity of ITD sensitive cells recorded from a single animal the place code was constructed across multiple experiments with cells having $\mathrm{CF}<500 \mathrm{~Hz}$. At any anteriorposterior location along this spatial map, peak ITDs varied $\pm 100 \mu \mathrm{s}$ from the regression line fit to the data provided. Yin and Chan also determined that cells have a characteristic delay (CD) firing maximally at roughly the same ITD across many frequencies. Additionally, MSO cells are sensitive to ITD of identical noise bands, but predictably, not sensitive to uncorrelated noise bands as these stimuli share no temporal similarities. General response characteristics of MSO cells including rate-level curves, response areas, tuning curves, spontaneous rate and PSTH classification were similar to response characteristics of their SBC excitatory inputs; however the tuning curves were broader, indicating convergence of inputs.

Sensitivity to ITD can be assessed by several different stimuli, each having certain advantages and disadvantages. Galambos utilized binaural clicks having different onset times, thus allowing him to test the ITD sensitivity of cells quickly and without regard to their characteristic frequency (Galambos et al., 1959). Because these stimuli are short and contain a broad spectrum of frequencies it is difficult to discern exactly whether cells are discriminating time or phase. Binaural beats are constructed by presenting pure tones to both ears that vary in frequency by " $\mathrm{x}$ " $\mathrm{Hz}$. Where $\mathrm{x}$ in this case determines the number of times the 2 tones will cycle through $360^{\circ}$ of phase relationships during a one second stimulus. If a $500 \mathrm{~Hz}$ tone is presented in the contralateral ear and a $501 \mathrm{~Hz}$ tone is presented in the 
ipsilateral ear these tones will cycle through their phase relationships once each second. The advantage of binaural beat stimuli is that all phase relationships can be explored more efficiently than stepping through phase angles across separate stimuli. Additionally, because the stimulus is ongoing, the onset response of the cell is removed for all phases except the initial starting phase, which is typically $0^{\circ}$. The most flexible stimuli used for the investigation of ITD sensitivity involve binaurally presented pure tones (the same frequency for both ears) or frozen noise bands whose onset times and, as a result, phase are varied in time. These stimuli allow onset responses to be analyzed separately from ongoing responses and the flexibility to present stimuli outside of the range that the subject typically encounters in its environment.

Another comprehensive study of ITD processing in the SOC, performed by Spitzer and Semple (1995), found 44 ITD sensitive neurons in the MSO column of gerbils. The majority of these cells phase-locked to monaural and binaural low frequency sounds, and those that did not were not sensitive to monaural stimuli. ITD sensitive cells responded similarly to previous studies showing that best ITDs generate summed (ipsi/contra) or facilitated responses and worst ITDs show suppression or occlusion of the response. A noteworthy result was that the average characteristic delay (CD), the ITD that generates maximum discharge rate, was 241 microseconds. The longest ITD an animal can naturally experience is dictated by the distance between the ears. A gerbil's ears are about $3 \mathrm{~cm}$ apart, meaning that the greatest naturally experienced delay is about \pm 132 microseconds; Joris referred to this interaural delay value as the ecological range of hearing, which varies as head size varies among species e.g. Fig. 36. More than a third of the calculated CDs were in the physiological range. If there were a place code, what purpose would an overrepresentation of best ITDs in the non-physiological range serve?

A study examining the sensitivity to ITDs by inferior colliculus (IC) cells provided more evidence of best ITDs outside of the ecological range. McAlpine et al. recorded from 520 ITD sensitive neurons in the IC of guinea pigs and found that the majority $(83 \%)$ of these cells had best ITDs for contralaterally leading stimuli and most of these had peak discharges at ITDs between 150 and 300 microseconds, outside the ecological range of guinea pigs (2001). McAlpine et al. proposed that a rate code, and not the Jeffress place code, is a better model for how ITD information is processed. The IC, then, was detecting the change in 
discharge rate generated by MSO neurons. The greatest change in rate was within the ecological range but, more specifically, at zero ITD, which is where animals have the greatest acuity. A direct comparison between response properties of IC and MSO cells cannot be made, but these data are relevant. Further refinement of the ITD signal at the level of the IC is most likely restricted to convergence of cells tuned to different frequencies and, while not implausible, it is unlikely that the Jeffress place code, so carefully constructed in the MSO would be modified to a population rate code that is found in the IC. Further refinement of the ITD signal at the level of the IC includes the convergence of cells tuned to different frequencies and consequently broadening of IC tuning curves. Despite converging inputs from the SOC, ITD windows of single IC neurons narrow resulting in increased ITD sensitivity concurrent to a reduction in the number of cells needed to represent azimuthal location of sound in space (Fitzpatrick et al., 1997).

Anatomical evidence suggests that the MSO receives sharply phase-locked inhibition from the MNTB and, I propose, the pvLNTB, with as yet unknown timing relative to the arrival time of excitation. In a now classic study, glycinergic inhibition was blocked in vivo, via strychnine iontophoresis, leading to a shift in the best ITD from a positive value (contralateral leading) in most cases outside of the ecological range, to a near zero ITD (Brand et al., 2002). This response property is in contrast to the Jeffress model, where cells are tuned so that best ITDs reflect the sound delays encountered in the animals environment. Modeling studies from the same lab demonstrated that inhibition arriving before excitation results in the shift of best ITD to non-physiological delays. Given that the $\mathrm{CN}$ has direct excitatory projections to the MSO it is difficult to explain how inhibition, originating in the MNTB and LNTB, could arrive before excitation. Anatomical specializations such as the calyx of Held and fast conduction times to and from the MNTB might be capable of compensating for an extra relay in ITD circuitry, but this remains conjecture in the absence of experimental evidence. Additionally, what function might the LNTB serve? In birds, where evidence for the Jeffress model exists, the inhibitory nuclei are necessary to preserve ITD sensitivity in the nucleus laminaris at increasing sound intensities. Might the LNTB serve the same purpose in mammals? Aim 3 addresses these issues of onset latencies of MNTB, LNTB, and the MSO in a computational model. These data will allow us to determine the relative onset of inhibition 
and excitation arriving at the cell body along with the shape and time-course of these waveforms.

In vitro intracellular experiments can provide unique information with regard to ion channel specialization, synapse dynamics and morphological constraints placed on synaptic integration and thus action potential generation. These data have been used to construct ever more realistic computational models that reflect the current, albeit changing, theories about how MSO cells code ITD. These studies have been driven by the question, "What specialization has the MSO and, more generally, the auditory system implemented to allow mammals to reliably navigate their auditory environment ?" These issues are addressed through computational modeling presented Chapter 3. 


\section{Interaural time difference}

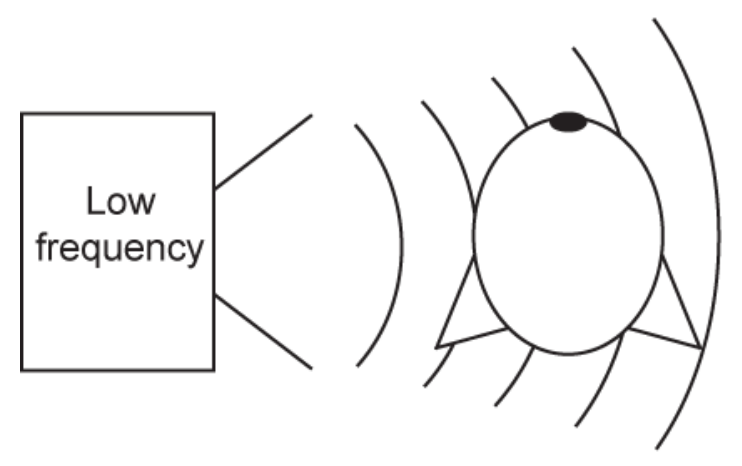

\section{Interaural level difference}

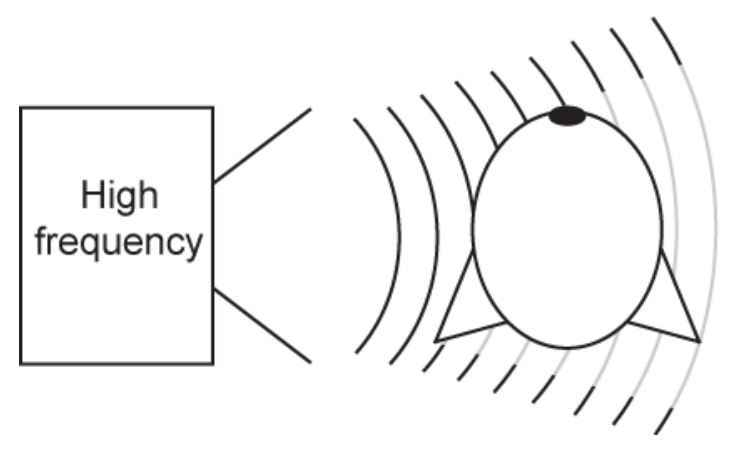

Figure 2-1.

Physical characteristics of sound relevant to the duplex theory of sound localization. When low frequency sound strikes an object (upper panel) the sound wave wraps around the object. Auditory circuitry codes the interaural time differences (ITD) or the time between the sound arriving at one ear relative to the other to localize the source. High frequency sounds cast and acoustic shadow (bottom panel) when striking an object. Our brains use the interaural level differences (ILD), between the two ears, to locate the source of the sound. 
Ipsi leading
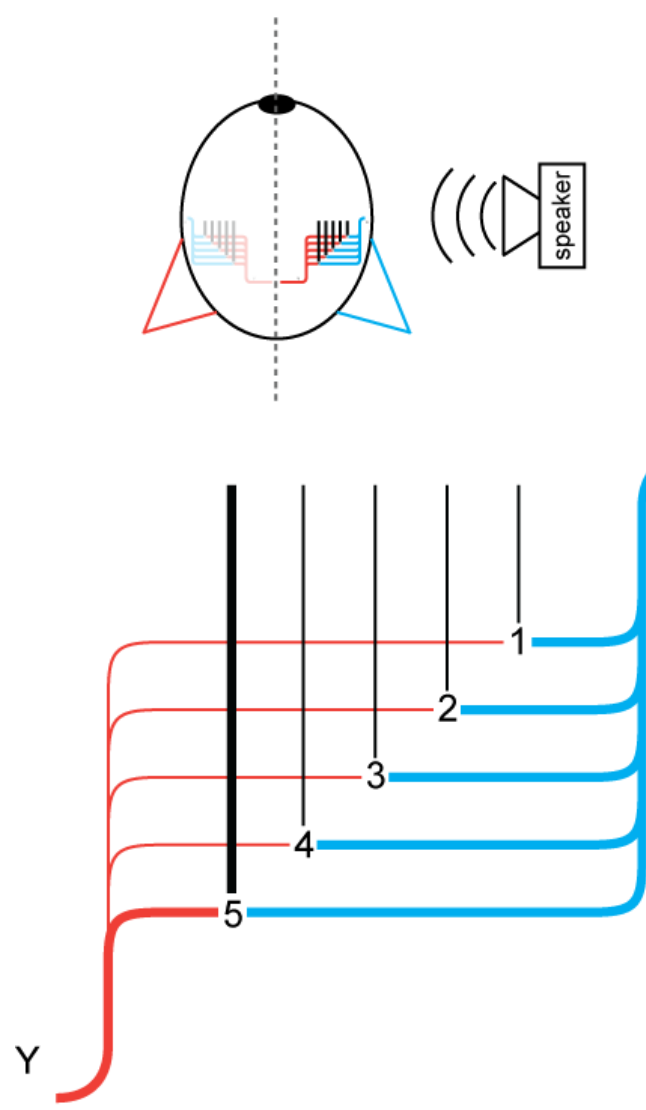

Contra leading

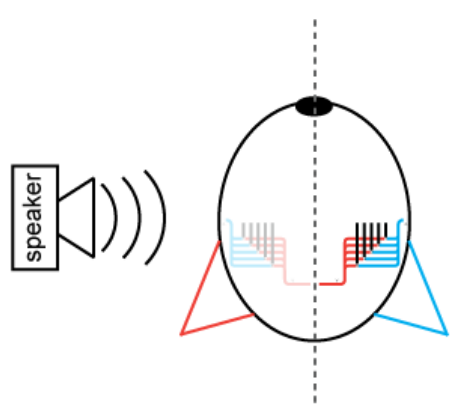

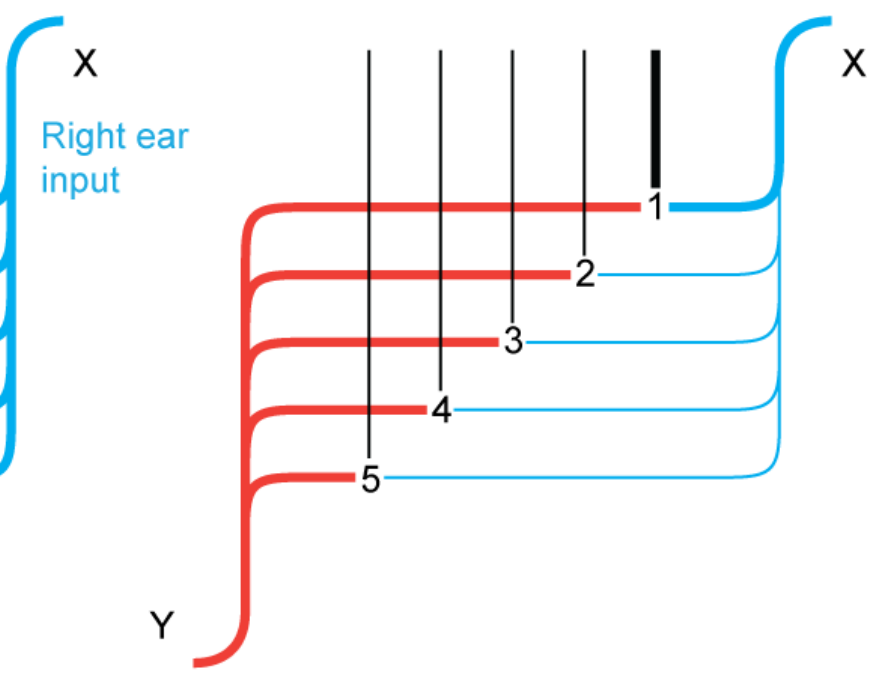

Left ear

input

Figure 2-2.

Jeffress coincidence detection circuit. Sub-threshold signals arrive at points $X$ and $Y$ depending upon the location of the stimulus in space (ipsilateral source in column 1 and contralateral source in column 2). These sub-threshold signals travel down delay lines reaching nodes $1,2,3 \ldots$ and $5,4,3 \ldots$ sequentially; only when signals arrive at a node in coincidence does the neuron respond and the signal propagated to higher auditory centers. 

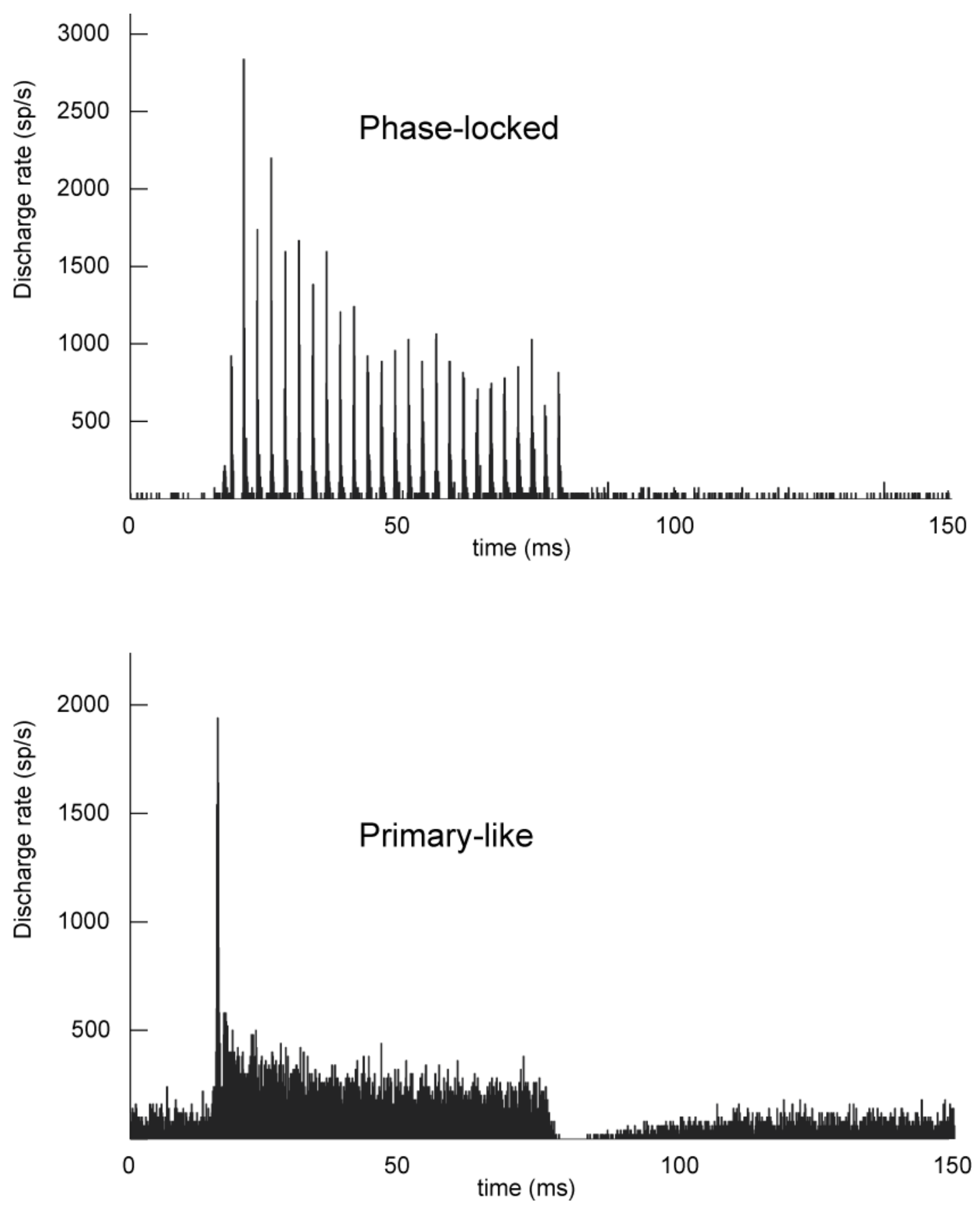

Figure 2-3.

Phase-locked and primary-like (PL) peri-stimulus time histograms (PSTHs). In cat cells having low characteristic frequencies (CFs), up to $4 \mathrm{kHz}$, phase lock to the period of a stimulus (top panel, CF = 400). Primary-like discharge patterns are observed in cells having higher CFs and are named for the similarities in response appearance to high frequency auditory nerve fiber responses (bottom panel, $\mathrm{CF}=12 \mathrm{kHz}$ ). 


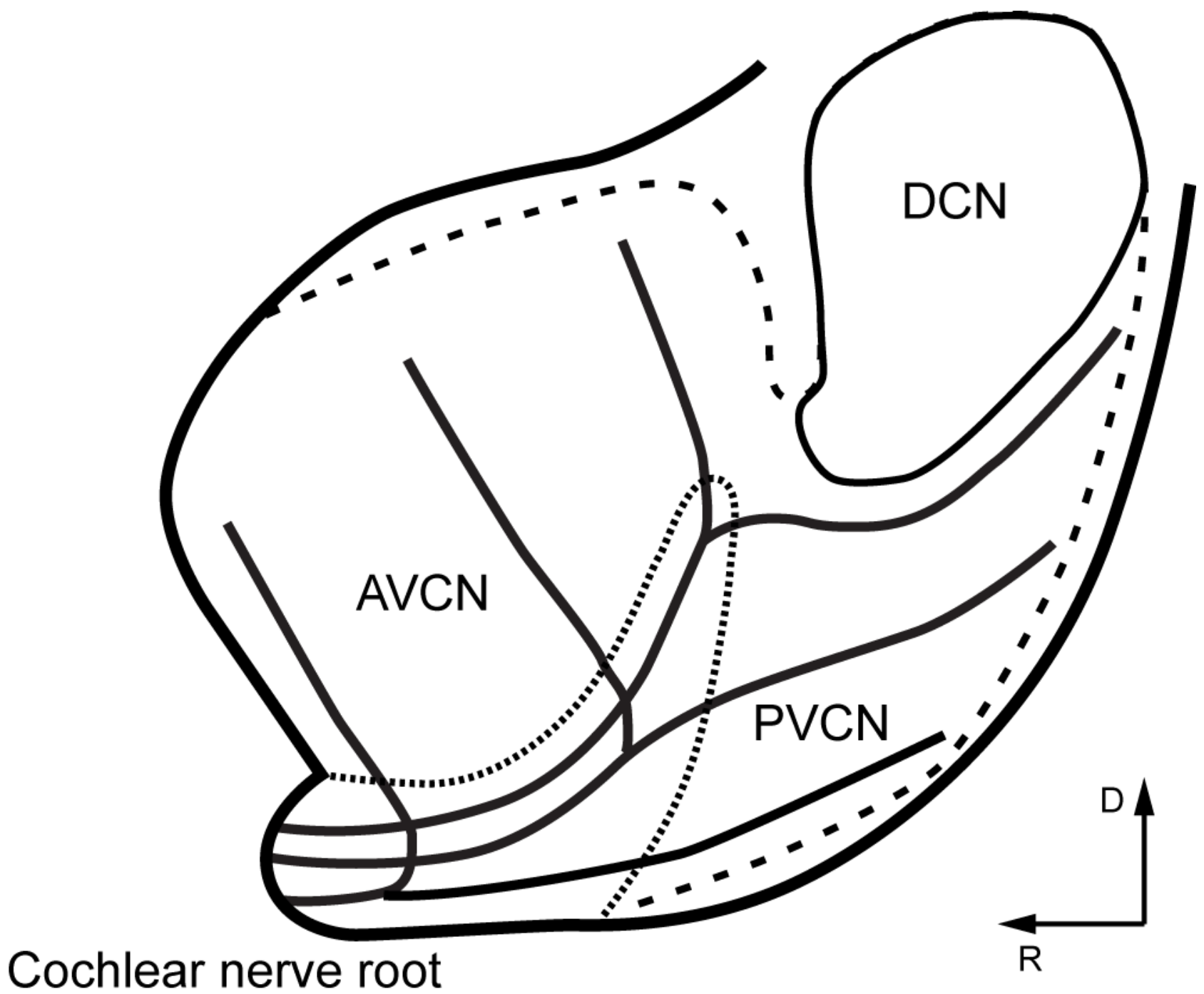

Figure 2-4.

Schematic diagram of the cochlear nucleus $(\mathrm{CN})$, viewed in the sagittal plane. The cochlear nerve root fibers innervate the 3 nuclei of the $C N$, the anteroventral (AVCN), posteroventral (PVCN) and dorsal (DCN). D= dorsal, R=rostral, adapted from Osen 1969. 


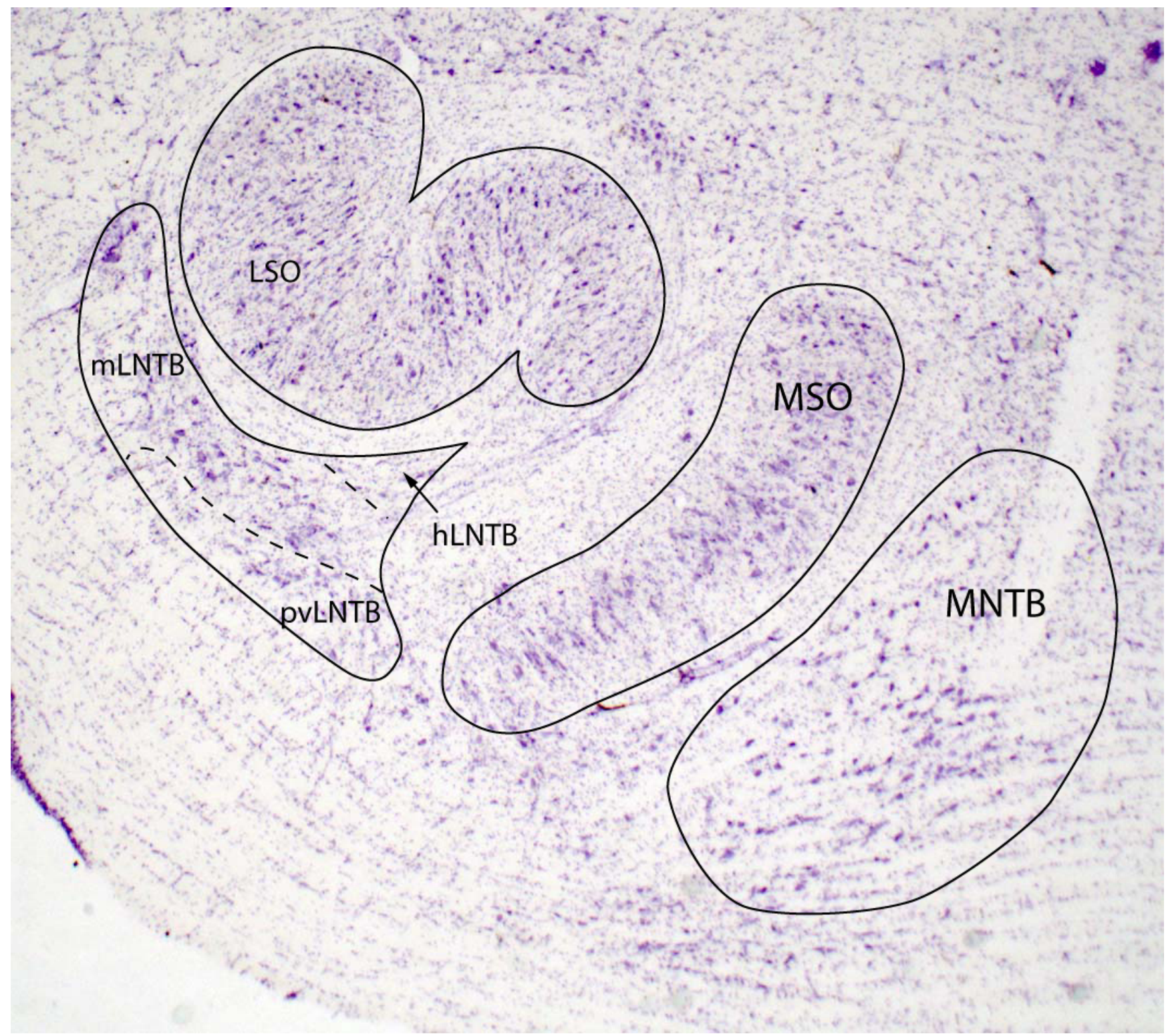

Figure 2-5.

The superior olivary complex. Nissl staining clearly shows the 3 most prominent nuclei of the SOC (solid lines), the medial superior olive (MSO), the lateral superior olive (LSO) and the medial nucleus of the trapezoid body (MNTB). Sub-nuclei of the lateral nucleus of the trapezoid body (LNTB) include the main (m)LNTB, the postero-ventral (pv)LNTB and the hilus (h)LNTB (separated with dashed lines). 


\section{Binaural Inhibition in the Medial Superior Olive}

The following chapter is a manuscript prepared for the Journal of Neuroscience 
Binaural Inhibition in the Medial Superior Olive of Cats: Circuit Structure and Functional Implications

Abbreviated or running title:

Binaural Inhibition in Medial Superior Olive

Jesse M. Thompson ${ }^{1,2}$, Paul B. Brown ${ }^{1,2}$, Ronald Millecchia ${ }^{1,2}$, George A. Spirou ${ }^{1,2,3}$

${ }^{1}$ Sensory Neuroscience Research Center, West Virginia University, Morgantown, WV, USA

${ }^{2}$ Physiology and Pharmacology, West Virginia University, Morgantown, WV, USA

${ }^{3}$ Otolaryngology-HNS, West Virginia University, Morgantown, WV, USA

Corresponding author:

George A. Spirou

One Medical Center Drive

PO Box 9303

Sensory Neuroscience Research Center

WVU School of Medicine

Morgantown, WV 26506

Email: gspirou@hsc.wvu.edu

Tel. 304-293-3490 Fax: 304-293-7182

12 figs, 4 tables, 31 pages

252 abstract, 590 intro \#discussion

Key words: ITD coding, MSO

No conflict of interest

Acknowledgements:

We thank Brian Pope for technical expertise and Philip Joris for critical review of the manuscript.

Grant sponsor and number: NIH/NIDCD 005035 to GS; NIH/NCRR P30 RR031155 to Center for Neuroscience 


\section{ABSTRACT}

Inhibition plays key roles in the neural representation of sound location by neurons of the medial superior olive (MSO), a cell group in the auditory brainstem of mammals. Contralateral, phase-locked inhibition originating in the medial nucleus of the trapezoid body (MNTB) has been studied physiologically and in biophysical models, but basic information about ipsilateral inhibitory projections to MSO is unknown. We studied ipsilateral inhibition in cats, which are sensitive to low frequency sound and have an interaural distance intermediate between more common rodent models and humans. Injection of retrograde tract tracer into low frequency regions of the MSO labeled a population of neurons in the lateral nucleus of the trapezoid body (LNTB) that match the cytology of MNTB cells. Stereological counts parsed by known frequency maps revealed a low ratio of 1 MNTB : 2 MSO neurons at frequencies $<5 \mathrm{kHz}$, and across all frequencies fewer LNTB than MSO neurons. We explored the consequences of bilateral, phase-locked inhibition in a biophysical model of a reconstructed MSO cell. Addition of LNTB inhibition could shift the peaks of interaural temporal delay (ITD) plots, relative to MNTB input only, by 0.1- $0.2 \mathrm{~ms}$ away from or toward a value of $0 \mathrm{~ms}$ ITD. LNTB inhibition also narrowed the ITD curve and increased its slope through the ecological range of hearing for the cat. Thus, bilateral inhibition may afford the binaural circuitry flexibility to tune the ITD curve and offer animals with larger heads mechanisms based upon the ITD curve peak detection and slope to encode sound location. 


\section{INTRODUCTION}

The temporal integration properties for individual neurons place constraints on the locations and functional properties, such as axonal conduction velocity, of cells that provide afferent input. Neurons of the medial superior olive (MSO) in the auditory brainstem of mammals impose very strict limitations on these parameters to perform coincidence detection on converging auditory information from both ears (Goldberg and Brown, 1969; Moushegian et al., 1975; Yin and Chan, 1990), whereby temporal disparities in binaural input on the order of tens to hundreds of microseconds yield large changes in discharge rate. The MSO receives bilateral excitatory projections from spherical bushy cells located somewhat distantly in the cochlear nucleus and bilateral inhibitory projections from the adjacent medial and lateral nuclei of the trapezoid body (MNTB, LNTB) (Stotler, 1953; Cant and Hyson, 1992). Little is known about the structure of the inhibitory circuitry, including basic information on the numbers and locations of inhibitory neurons relative to innervation targets in the MSO.

Cytological characteristics of MNTB and LNTB neurons suggest potential symmetries in the bilateral inhibitory projection. The MNTB is a source of glycinergic inhibition driven by large calyx of Held nerve terminals of globular bushy cells (Morest, 1968; Friauf and Ostwald, 1988; Smith et al., 1991). The LNTB consists of three sub-nuclei; neurons in the largest two subnuclei, the main $(\mathrm{m})$ and posteroventral (pv) LNTB, are mostly glycine and/or GABA immunoreactive (Spirou and Berrebi, 1996, 1997). Like the MNTB, the pvLNTB contains cells that are strongly glycine-immunoreactive and contacted by large terminals of globular bushy cells (Warr, 1969; Tolbert et al., 1982; Smith et al., 1991; Spirou and Berrebi, 1997). A primary goal of this study was to characterize innervation of MSO from LNTB subnuclei in comparison to MNTB projections.

Modeling studies have shown the importance of temporally precise, short duration excitation and inhibition to sensitivity of firing rate to interaural temporal delay (ITD). Current debate compares the roles for peaks of ITD curves, which may be differentially tuned across the MSO cell group into a place code (Jeffress, 1948), and slopes of ITD curves, whereby monotonic changes in rate are reported by all MSO neurons as a population rate code (McAlpine et al., 2001; Brand et al., 2002). Most models of MSO neurons to date have incorporated a single class of inhibitory inputs, assumed to originate from MNTB, mixed with 
bilateral excitatory inputs to explore functional roles of inhibition, but roles of a second inhibitory input originating from LNTB have received little attention (see Leibold, 2010). Because MSO neurons receive bilateral inhibitory inputs, and because of the confirming anatomical data we report here, we explored, in a computational model, the consequences of two distinct inhibitory projections to single MSO neurons. To do so, we reconstructed a neuron from the low frequency $(<1 \mathrm{kHz})$ region of the $\mathrm{MSO}$, developed a compartmental model using recent information on biophysical properties across cellular compartments (Mathews et al., 2010; Scott et al., 2010) and applied independent excitatory and inhibitory inputs through a local circuit that matched the physiological features of these inputs (Joris et al., 1994b). We show that a second inhibitory input, temporally locked to excitatory inputs, could increase slopes and sharpen peaks of ITD curves and exert small shifts (100-200 $\mu$ s) of the peak firing rate to longer ITD values. A second inhibitory input, then, provides the binaural circuit with an additional mechanism to adjust ITD curve parameters in a manner that is applicable across species and factors into both place and population rate mechanisms for ITD coding. 


\section{METHODS:}

\section{Surgical Procedures}

Twelve colony-raised adult cats (Harlan, Minneapolis, MN, USA), between 9 and 24 months of age, were used for these experiments. Anatomical and in vivo electrophysiology experiments were carried out simultaneously in these animals; however, only anatomical data are presented here. All procedures were in accordance with the National Institutes of Health Guide for the Care and Use of Laboratory Animals, and all protocols were approved by the Institutional Animal Care and Use Committee of the West Virginia University School of Medicine.

Animals were decerebrated after initiation of anesthesia with xylazine $(10 \mathrm{mg}$; Phoenix Pharmaceuticals, St. Joseph, MO, USA) and ketamine (10 mg/kg; Fort Dodge Animal Health, Fort Dodge, IA, USA); maintenance doses of $20 \mathrm{mg}$ ketamine were administered as needed. After a tracheal cannula was inserted to maintain a clear airway, a $2.25 \mathrm{~cm}^{2}$ section of the skull overlying the auditory cortex was removed to permit aspiration of a portion of the cortex and hippocampus overlying the midbrain. Decerebration was achieved by transecting the neural axis through the rostral portion of the superior colliculus by aspiration under visual control. Anesthesia was discontinued to permit recording of neural activity without pharmacologic influence. The animal was secured in a stereotaxic frame and the head was rotated $20^{\circ}$ forward from the Horsley-Clark plane to align the dorsal surface of the brainstem close to the horizontal plane. A dorsal approach to the superior olivary complex (SOC) was achieved by removal of a portion of the supra-occipital bone and aspiration of the underlying cerebellum to expose the floor of the fourth ventricle. A sterile saline agar (1\%) solution was applied to the brain stem to minimize cardiac and respiratory pulsations. Ear bars were placed in the ear canals and the bullae were vented with a length of small-bore polyethylene tubing. Temperature was maintained at $36-38{ }^{\circ} \mathrm{C}$ using a rectal probe thermometer and electric heating pad. Sterile lactated Ringer's solution (Baxter Healthcare Corporation, Deerfield, IL, USA) was injected subcutaneously regularly to preserve fluid volume.

\section{Tract Tracer Placement}


Electrode placement was achieved using brainstem surface landmarks. In nine animals, borosilicate glass micropipettes (tips 2 - 4 microns) filled with biotinylated dextran amine (BDA, low molecular weight, $10 \%$ in normal saline; Vector Laboratories, Burlingame, CA, USA) were placed $0.5-1 \mathrm{~mm}$ lateral of midline and in line with the caudal edge of the cerebellar peduncle. To align the track parallel to the dorsomedial to ventrolateral axis of the $\mathrm{MSO}$, the tip of the electrode was angled $30^{\circ}$ rostrally from vertical in the rostral-caudal axis and rotated $45^{\circ}$ laterally. Typically, neurons sensitive to auditory stimuli were encountered at recording depths between 5 and $9 \mathrm{~mm}$. Initial identification of cells within the nuclei of the SOC was made by examining responses to monaural and binaural sounds presented to both ears. In general, MNTB neurons are stimulated by contralateral sound, LNTB neurons are stimulated by ipsilateral sound, and MSO units produce the highest discharge rates when sound is presented to both ears at their characteristic delay (for most MSO cells, the delay in stimulus onset between the two ears which results in the greatest discharge rate).

Electrodes were advanced using a piezoelectric microelectrode positioner (Burleigh, Fishers, NY, USA) and recording depths for isolated units and BDA injections were noted. BDA was iontophoresed into putative MSO regions when isolated neurons responded with excitation to monaural stimuli presented in both ears or when click evoked responses produced ABRs characteristic of MSO recordings (Guinan et al., 1972a). BDA injections were performed using small positive currents $(0.6-2 \mu \mathrm{A}$; Stoelting, Wood Dale, IL, USA) and a $50 \%$ duty cycle every 14 seconds and continued for 10 minutes. Physiological recordings (not reported here) were typically continued for $36-48$ hours following BDA iontophoresis. Animals were then perfused transcardially with $0.9 \%$ saline, $\mathrm{pH} 7.2$ at room temperature (all chemicals from Sigma, St. Louis, MO, USA, unless otherwise noted), followed by fixative solution containing $4 \%$ para-formaldehyde and $0.1 \%$ glutaraldehyde (Polysciences, Warrington, PA, USA) in $0.1 \mathrm{M}$ sodium phosphate buffer. The head was covered with ice for $1-2$ hours post-fixation, and post-fixed overnight prior to removal of the brain.

\section{Tissue Processing}

The brains were carefully dissected from the skull. A section of tissue including the brainstem and midbrain was removed and cryoprotected in a $30 \%$ sucrose solution for $2-5$ days before slicing on a freezing stage microtome (Microm $\mathrm{GmbH}$, Walldorf, Germany). 
Tissue was sectioned in the transverse plane at 45 or $50 \mu \mathrm{m}$ thickness. Tissue sections were collected in $0.5 \mathrm{M}$ Tris-HCl buffer, $\mathrm{pH} 7.6$, pre-incubated in hydrogen peroxide (2\%) and methanol (6.6\%) $0.5 \mathrm{M}$ Tris solution to reduce endogenous peroxidase activity, rinsed in 0.5 $\mathrm{M}$ Tris and washed in $\mathrm{x}-100$ Triton (.75\%) $0.5 \mathrm{M}$ Tris to permeabilize cell membranes. BDA was visualized using an Elite ABC kit (Vector Laboratories, Burlingame, CA, USA) and incubated up to $30 \mathrm{~min}$ in hydrogen peroxide and diaminobenzidine, intensified by the heavy metals nickel and cobalt. After rinsing in Tris buffer, all sections were dry mounted from a gelatin alcohol solution. Sections were counterstained with Cresyl Violet. Every eighth section was unstained to promote localization of labeled structures.

\section{Electrophysiology}

Particular to this report, stimuli were only used to characterize the characteristic frequency (CF) of an area or an isolated single unit before injections were made, so only a limited description of our stimulation and recording system is provided. Stimuli were designed and generated with System 3 hardware and Real-time Processor Visual Design Studio software (Tucker Davis Technologies). Sounds were delivered to the animals through a closed acoustic system via custom-built amplifiers and speakers (Sokolich 1977) calibrated at the beginning of each experiment with a $1 / 2$ inch microphone (model \# 4134, Bruel \& Kjaer) attached to a probe tube. Stimuli were generated at 100 or $200 \mathrm{kHz}$ sampling rate; electrode signals were digitized at $25 \mathrm{kHz}$. The search stimuli (100 ms in duration) consisted of a Gaussian noise or pure tones presented binaurally at $1 \mathrm{~Hz}$. After the CF and threshold were determined audio-visually, stimulus parameters and stimulus types were set using custom C code; stimulus presentation and data collection were managed with Brainware (Jan Schnupp, Oxford, England).

3D Reconstruction of Electrode Tracks, Cell Groups and Injection Sites Injection sites and electrode tracks were reconstructed in three dimensions at 10X or 20X using Stereo Investigator software (MicroBrightfield, Williston, VT, USA) interfaced with a motorized stage and Z-position readout on an upright Leica Leitz DMRB microscope (Leica Microsystems, Wetzlar, Germany). The surface of the tissue, the midline, and outlines of the lateral superior olive (LSO), MSO and MNTB were traced from every fifth slide. Intervening 
sections were used to trace salient features of the BDA injection and electrode tracks to assure accurate reconstructions. Tracks were identified and reconstructed by outlining glial scars created by the electrode penetrations and electrical lesions. Depths of BDA injections and lesions relative to the brainstem surface as recorded during the experiment were plotted on the reconstructed brain to calculate a tissue shrinkage factor.

Analysis of Retrograde Labeling

BDA-labeled cells were identified from surrounding cells by their dark brown or black staining and were assigned to specific nuclei based upon a 3D atlas defined previously in our laboratory (Spirou and Berrebi, 1996). Brains from each experimental animal were reconstructed in 3D. The orientation of the MSO was normalized in the rostro-caudal dimension so that injection site locations could be compared across experiments. The linear distances from all labeled cells to the center of the BDA injection site were calculated for each experiment. These distances were pooled across experiments to yield a distribution of distances for neurons located in the MNTB, and the main (mLNTB) and posteroventral (pvLNTB) subdivisions of the LNTB (see Results for explanation of anatomical regions) to the injection sites in the MSO.

\section{Stereology}

We employed unbiased stereology techniques to count cells in the MNTB, MSO and LNTB. Four animals, all males and of similar weight $(4.1 \pm 0.17 \mathrm{~kg})$, were used for these measurements. To ascertain the appropriate sampling frequency for these counts, we utilized an oversampling method for the optical disector (Slomianka and West, 2005). After outlining each cell group at $5 \mathrm{X}$ or $10 \mathrm{X}$, we set the counting frame and optical disector to the same $\mathrm{X}-\mathrm{y}$ dimensions measuring $60 \times 60 \mu \mathrm{m}$, so that the entire profile of each cell group in the tissue section was sampled. We used a 100x oil immersion objective lens (NA 1.4) to count cell nucleoli (see Reconstructions, Electrode tracks and Injection sites for microscope details). Typical sampling strategies dictate counting at least 10 tissue sections and at least 10 frames per section to yield $100-200$ neuron counts. We counted every frame in every $4^{\text {th }}$ section, thereby counting 22 of 82 sections in the MNTB and 21 of 80 sections in the MSO, sampling 7858 and 4925 counting sites in the MNTB and MSO, respectively. For the sub-nuclei of the 
LNTB, which have unusual shapes, we counted every frame in every other section (mLNTB, 34 of 66 sections; pvLNTB, 35 of 68 ; hLNTB, 13 of 24) for a total of over 9000 counting sites. The goal of these procedures was to quantify both counting error (determined from the sampling strategy) and biological error (the existing variability in biological samples). For this purpose, we first used the Gunderson-Jensen estimator to calculate coefficient of error (CE),

$$
\begin{aligned}
& C E(Q)=\frac{\sqrt{\left(3\left(A-S^{2}\right)-4 B+C\right) \times \alpha+S^{2}}}{Q^{-}} \\
& A=\sum_{i=1}^{n}\left(Q_{i}^{-}\right)^{2} \\
& B=\sum_{i-1}^{n-1} Q_{i}^{-}-Q_{i+1}^{-} \\
& C=\sum_{i-1}^{n-2} Q_{i}^{-}-Q_{i+2}^{-}
\end{aligned}
$$

where $A, B$ and $C$ serve to examine the smoothness in count distribution of 3 consecutive sampled sections. $Q^{-}$represents the nugget or the sum of the counts for all sections of a particular region of interest, where $S^{2}$ is nugget variance, and $\alpha$ is smoothness of the entire sampled tissue. We calculated CE for the 2 extremes of smoothness (smoothness factors of 0 and 1 ) where $\alpha=1 / 12$ and $1 / 240$ for $C E_{0}$ and $C_{1}$, respectively (supplemental table 1). For a more detailed description of this analysis see Gundersen et al. (1999). The coefficient of variation $(\mathrm{CV})$ is dependent on both $\mathrm{CE}$ and biological variation $(\mathrm{BV})$ and is expressed as: $C V^{2}=B V^{2}+$ mean $C E^{2}$ (Gundersen and Jensen, 1987). meanCE was calculated as the average $C E$ of the sub-sampled tissue. Thus, by over-sampling our data, $C E$ approaches 0 and consequently $C V^{2}=B V^{2}$. By sub-sampling our oversampled data we created populations that should have the same BV. We found that sampling every $4^{\text {th }}$ section in the pvLNTB and $m L N T B$ and every $8^{\text {th }}$ section in the MSO and MNTB was adequate for accurate estimates and led to BV values that were roughly equal to $\mathrm{CV}$. Through systematic sub-sampling of our over-sampled tissue we concluded that an accurate count could be attained by analyzing at least 10 sections for the MNTB and MSO and at least 17 sections for the pvLNTB and mLNTB, counting every tissue frame on those sections.

Linear Summation Model of Inhibitory Synaptic Inputs

We explored the effects of inhibitory cell location and action potential conduction velocity on inhibitory post synaptic potential (IPSP) latencies at the MSO using a linear 
summation model. Euclidian distances were measured from labeled cells to the center of the BDA injection site in order to estimate the arrival of IPSPs from the MNTB, mLNTB and pvLNTB. Five conduction velocities $(1.25,2.5,5,10,20 \mathrm{~m} / \mathrm{s})$, encompassing the probable physiological range of axonal conduction velocity for these cell types, were examined across 3 conditions: MNTB and LNTB activated simultaneously; MNTB leading LNTB by $400 \mu \mathrm{s}$; MNTB lagging LNTB by $400 \mu \mathrm{s}$. Individual cell distances were binned (100 $\mu \mathrm{m}$ bins) and convolved with an IPSP modeled as an alpha function of the following form:

$$
\alpha=\mathrm{V}_{\mathrm{rmp}}+\mathrm{V}_{0} \times\left(\left(\mathrm{x}-\mathrm{T}_{0}\right) / \tau\right) \times \exp \left(1-\left(\mathrm{x}-\mathrm{T}_{0}\right) / \tau\right)
$$

Where $V_{r m p}$ is the resting membrane potential, $T_{0}$ is the start time, $\tau$ is the time constant, $x$ is time and $V_{0}$ is the scaling factor. We modeled inhibitory postsynaptic potentials (IPSPs) (Chirila et al., 2007), using the following values: $\tau=0.38 ; V_{\mathrm{rmp}}=-70 \mathrm{mV} ; \mathrm{V}_{0}=-0.95 ; \mathrm{T}_{0}=0.1$. These individual IPSPs in MNTB, mLNTB or pVLNTB were grouped by source, onsets adjusted by the conduction velocity and summed, resulting in compound IPSPs that represented inhibition arriving to MSO cells from each inhibitory nucleus or subnucleus. Calculations were performed using Matlab (MathWorks, Natick, MA).

\section{NEURON Model}

Cell morphology. A cat MSO cell was reconstructed at high resolution (100X oil immersion, NA 1.4) in Neurolucida (MicroBrightfield, Williston, VT, USA) using variable step size. The cell, which was located in the dorsal region of the MSO, had an axon emanating from the middle of the soma. The soma was approximately $40 \mu \mathrm{m}$ long, $18 \mu \mathrm{m}$ in diameter at its center and tapered into primary dendrites located at the medial and lateral poles (Fig. 31A). These dendrites measured $3.6 \mu \mathrm{m}$ and $4.9 \mu \mathrm{m}$ diameter at their base, were oriented approximately in the coronal plane and could be followed across several tissue sections. At a distance of approximately $100 \mu \mathrm{m}$ from the cell body, each proximal dendrite branched and each branch ultimately terminated with tertiary branches having diameters $<2 \mu \mathrm{m}$. Two other dendrites emanated rostrally or caudally from the cell body, could not be traced across sections and were removed from the model cell (grey structures in Fig. 3-1A). The morphological model was converted into a NEURON-ready model using CVAPP (Robert Cannon) and the axon was replaced with a model axon (Spirou et al., 2005). The model axon consisted of an initial heminode, approximately $36 \mu \mathrm{m}$ long and $4 \mu \mathrm{m}$ in diameter followed by 
a myelinated section $4 \mu \mathrm{m}$ in diameter and $1 \mathrm{~mm}$ long followed by a node of Ranvier $4 \mu \mathrm{m}$ in diameter and $2.5 \mu \mathrm{m}$ in length. Four additional myelinated axon/node of Ranvier sequences comprised the axon, with a regular $1 \mathrm{~mm}$ distance between nodes. Simulations were performed using NEURON (Hines and Carnevale, 1998).

Ion channels. The model was populated with ion channels and cellular distributions demonstrated for MSO neurons (Rothman and Manis, 2003a; Mathews et al., 2010; Scott et al., 2010). The soma, dendrites, axon hillock and nodes had a membrane capacitance of 1 $\mu \mathrm{F} / \mathrm{cm}^{2}$ and axial resistance of $200 \mathrm{ohm} \cdot \mathrm{cm}$. The myelinated sections had a membrane capacitance of $0.1 \mu \mathrm{F} / \mathrm{cm}^{2}$ and an axial resistance of $200 \mathrm{ohm} \cdot \mathrm{cm}$. The soma contained an array of ionic conductances $\left(g_{K l t}, g_{K h t}, g_{h}, g_{N a}\right.$; see appendix. Axon parameters were based upon models of bushy cells (Rothman and Manis, 2003b; Spirou et al., 2005). The initial segment of the model axon had a $\mathrm{Na}$ conductance of $0.1\left(\mathrm{~S} / \mathrm{cm}^{2}\right)$ that increased linearly to $0.6\left(\mathrm{~S} / \mathrm{cm}^{2}\right)$ at its final compartment where it attached to the first myelinated compartment. Other ionic conductances $\left(g_{\mathrm{lt}}, \mathrm{g}_{\mathrm{ht}}, \mathrm{g}_{\mathrm{h}}, \mathrm{g}_{\mathrm{leak}}\right)$ did not vary with location in the initial segment. The four myelinated sections of the axon had only passive conductance interspersed with nodes of Ranvier containing the entire assortment of ionic conductances, except $\mathrm{g}_{\mathrm{h}}$. In the nodes, Na conductance $\left(G_{\mathrm{Na}}\right)$ was adjusted so that peak action potential depolarization measured at the last axonal node varied from -10 to $0 \mathrm{mV}$. Excess $\mathrm{G}_{\mathrm{Na}}$ in the initial segment, soma or nodes widened the ITD window and shifted the peak discharge rate to 0 ms ITD (Zhou et al., 2005). The action potentials were counted from voltage waveforms generated at the last node of the axon. Characteristic equations for each conductance are listed in the appendix.

IPSPS and EPSPs. Individual excitatory and inhibitory inputs were modeled as phaselocked activity patterns governed by a Gaussian distribution model for spherical bushy cell activity (Zhou et al., 2005). We examined the effect that excitatory and inhibitory input location had on features of excitatory postsynaptic potentials (EPSPs) and IPSPs recorded at the soma. Locations of inhibitory inputs along the medial-lateral axis of the relatively electrically homogenous soma resulted in negligible differences in the IPSP height and are not shown. Excitatory inputs were distributed along each primary dendrite in a passive model of the cell membrane. To explore the effect of synaptic locations, we varied the location of a 
single excitatory synaptic input from 0.1-1.0 (fractional length) of the distance from the cell body to the first dendritic branch point (tics in Fig. 3-1A "cell model input location"). As expected, inputs located nearest to the soma generated the largest amplitude EPSPs. In each dendrite a uniform decrease in depolarization of the resting membrane potential was observed as the input was moved further away from the soma (Fig. 3-1B). Minimal differences in excitatory synaptic input occurred from each side (Fig. 3-1C).

Given the importance of frequencies less than $500 \mathrm{~Hz}$ to human hearing and sensitivity of cats in this frequency range, we modeled responses to a $250 \mathrm{~Hz}$ stimulus, because the long period ( $4 \mathrm{~ms}$ ) permitted us to describe synaptic integration without interactions across stimulus cycles. We considered also that a long stimulus period would distribute spikes more broadly in time and would not limit the effects of inhibition on shifting the peaks of ITD curves. Each excitatory and inhibitory input was modeled as a Gaussian distribution across stimulus period, and the standard deviation was adjusted to yield inputs with high vector strength $(R=0.954 \pm 0.004)$ to reflect published data for spherical and globular bushy cells and MNTB cells in cats (Fig. 3-1 D, E) and similar characteristics of low frequency globular and MNTB neurons (Joris et al., 1994b). Each input was constrained to a maximum of one discharge per stimulus period and the resulting interspike intervals had Poisson-like distributions (Fig. 3-1E). Simulations were carried out over 1000 stimulus cycles (e.g. 4 seconds at $250 \mathrm{~Hz}$ ) so that enough action potentials could be collected to discriminate successive ITD time points. Onset times of inhibition and excitation were varied at $100 \mu$ S ITD intervals. 


\section{RESULTS}

Stereological counts of MSO, MNTB and LNTB

As an initial estimate of the relative influence of inhibitory cell groups on binaural processing by MSO neurons, we counted the numbers of neurons in the MNTB, LNTB and MSO using the optical fractionator method of unbiased stereology. Counts of LNTB neurons were segregated by subnuclei, defined as the main $(m)$, posteroventral $(p v)$ and hilus $(h)$ LNTB (Spirou and Berrebi, 1996). The MNTB and MSO cell groups are relatively uniform in shape along the rostral-caudal axis, but LNTB subnuclei have more complex geometry. Therefore, we oversampled counts in one animal to determine the optimal sampling frequency for each cell group or subnucleus (see Methods). The MNTB contained slightly more neurons than the MSO (9379 \pm 1336 vs. $7831 \pm 828$; mean $\pm s d, n=4)$ and about 1.7 times the number of LNTB neurons $(5,450 \pm 734)$. These estimates were larger than published values in cats for MNTB and MSO (Moore and Moore, 1971) and smaller than previous studies of LNTB (Spangler et al., 1987; Spirou and Berrebi, 1996) using nonstereological techniques. Approximately $70 \%$ of LNTB neurons were located in the mLNTB (3839 \pm 552 ), about $25 \%$ were located in the pvLNTB (1409 \pm 348$)$, and very few were located in the hLNTB $(202 \pm 64)$. The rank order for numbers of cells (MNTB > MSO > $m L N T B>$ pvLNTB) was the same for each animal.

Both the MSO and MNTB exhibit different arrangements of cells along their tonotopic axes. The density of cells is lower in the dorso-lateral (low frequency region) than ventromedial (high frequency region) MNTB (Fig. 3-2 A-C). The width of MSO, especially rostrally, is greater at dorsal (low frequency region) than at more ventral (higher frequency) locations, where cells can form a single column (Fig. 3-2 D-F). Tonotopic maps are published for sound-evoked activity in cat MSO and MNTB (Guinan et al., 1972a), but tonotopic organization of the LNTB is less certain (Tsuchitani, 1977), especially given the complex geometric arrangement of the pvLNTB (Spirou and Berrebi, 1996). The MSO was parceled into frequency bands ( $<2 \mathrm{kHz}, 2-5 \mathrm{kHz},>5 \mathrm{kHz}$ ) that occupied approximately equal thirds in distance along the dorsal-ventral axis in the coronal plane of section. Corresponding frequency partitions were made in the MNTB. Even given the slightly larger number of neurons in the MNTB than MSO, there was a ratio of about 1 MNTB: 2 MSO neurons in the frequency bands < 2 and $2-5 \mathrm{kHz}$ (Table 3-1; MNTB, 30\%; MSO, $73 \%$ for CF $\leq 5 \mathrm{kHz}$ ). 
Therefore, branching in axonal projections is required to deliver a large number of inputs from the MNTB to individual MSO neurons.

Retrogradely-labeled neurons were located in the MNTB and in both major LNTB subdivisions

We used small amplitude iontophoresis currents $(0.6-2 \mu \mathrm{A})$ to create injection sites of biotinylated dextran amine (BDA) contained entirely within the MSO (injection sites ranged from $50-200 \mu \mathrm{m}$ diameter) to identify cells projecting to localized regions of this cell group. In 7 cases, injections into the MSO were contained entirely within its cellular borders as defined using Nissl stain. These cases were classified according to their dorsal-ventral and rostral-caudal location by dividing each axis into thirds ( $d-v$ orientation is illustrated for 3 cases in Fig. 3-3 A-B; locations of all 7 cases shown in sagittal plane in Fig. 3-5A). Of the 7 total cases, 4 cases were located dorsally (1 dorso-rostral (d-r); 2 dorso-middle (d-m); 1 dorso-caudal ( $d-c)$ ), 2 cases mid-frequency $(2 \mathrm{~m}-\mathrm{m})$ and 1 case ventrally $(1 \mathrm{v}-\mathrm{c})$. In four instances where we isolated single units at the injection site, their CF corresponded to the tonotopic map of the MSO as defined by Guinan et al. (1972a).

Because injection sites were well-contained, small numbers of neurons were labeled retrogradely in SOC cell groups. In 5 of 7 cases, as few as 11 and as many as 111 cells were retrogradely labeled in the MNTB. The other 2 cases, described below, labeled only a few cells in either the MNTB or LNTB. The topography of MNTB-MSO innervation was illustrated by the more medial location of labeled MNTB neurons with more ventral locations of the BDA injection site in the MSO. Even within the restricted frequency range in $\mathrm{MSO} \leq 5 \mathrm{kHz}$, ventral shifts in the injection site led to small shifts in the locations of labeled MNTB cells (Fig. 3-3 BF).

Given the similarity in cytological features of MNTB and pvLNTB neurons and the likely innervation of pvLNTB neurons by globular bushy cells via large excitatory nerve terminals (Warr, 1969; Spirou et al., 1998), one of our key objectives was to determine whether pvLNTB neurons innervate the MSO. As depicted in a stereo-pair image of a 3D rendering of the SOC, labeled cells are located in both the pv and $\mathrm{mLNTB}$ (dots in blue and pink areas of LNTB in Fig. 3-4A labeled from injection site in Fig. 3-3A). A horizontal view of cell groups and labeled cells from this injection reveals that most labeled pvLNTB cells are located in the caudal cluster of this cell group (Fig. 3-4B, compare left and right images). A 
camera lucida depiction of a caudal section shows labeled neurons in the pvLNTB (10 labeled cells in this section, Fig. 3-4C). A summary across cases reveals that the number of cells labeled in the pvLNTB were roughly equal to or greater than the number in $\mathrm{mLNTB}$ (Table 3-2). Innervation of MSO by pvLNTB neurons occurred with injections in the dorsal and middle regions. The ventral injection was very small and labeled only few MNTB neurons, so is not a good indicator of whether pvLNTB neurons innervate the ventral MSO. These data indicate bilateral input to MSO neurons by phase-locked inhibition from MNTB and, based on cytological features (see Introduction), phase-locked inhibition from pvLNTB.

Given the importance of timing of excitatory and inhibitory inputs to ITD processing by MSO neurons, we noted that the range of distances of MNTB and LNTB neurons from the injection site could differ. Even in the same coronal tissue section, labeled MNTB neurons were separated by up to $1 \mathrm{~mm}$ in the same frequency lamina. Therefore, we quantified the relative locations of inhibitory cell populations labeled from single injection sites. For the dorsal MSO injection site shown in Fig. 3-3A, most labeled cells in the MNTB were located at the same coronal plane and along the extent of the lowest isofrequency lamina (horizontal image plane in Fig. 3-4 A, B). A horizontal view of the SOC in this animal shows that in the LNTB, labeled cells were located predominantly at or caudal to the injection site (Fig. 3-4B). The two smallest injection sites were located laterally or medially in the MSO. The medial site labeled one MNTB neuron but no LNTB neurons (\#5 in Fig. 3-4E); the lateral site labeled 3 LNTB (all pvLNTB) neurons but no MNTB neurons (\#7 in Fig. 3-4E; note that these two cases are not reported in Table 3-2).

One important concern with tract tracing experiments is potential labeling of fibers of passage that do not innervate the injection site. We minimized this concern by using micropipettes with small tip diameter $(2-5 \mu \mathrm{m})$ to decrease the chance of severing axons by the pipette, using small iontophoresis currents to limit electroporation of neural structures and, perhaps most important, by approaching the MSO dorsally to avoid penetrating the MNTB or LNTB. As a control, when injection sites were located in the fiber capsule surrounding the MSO, axons were rarely labeled and these could only be traced over short distances. Anterograde tracing from MNTB and LNTB is confounded by axons of spherical bushy cells, the excitatory projections to MSO, passing through these cell groups. Therefore, we performed large injections dorsal to (in reticular formation and dorsomedial periolivary 
nucleus (dmPO)) and medial to (in MNTB and VNTB) MSO to capture potential projections to or through these territories that also passed through $\mathrm{MSO}$, and we investigated cases where smaller injections missed the MSO. In two cases, several injection sites were made in the same animal to encompass large areas including the reticular formation, dmPO, MNTB and VNTB, and labeled a total of 1 pvLNTB cell (Fig. 3-4E). In three other cases, injection sites confined to the MNTB did not label any LNTB cells. Therefore, if LNTB cells were labeled as fibers of passage from injection sites confined to the MSO, their axons did not extend through the MSO into the immediately surrounding dorsal or medial cell territories.

\section{Estimated neural delays from MNTB, LNTB to MSO}

The distributed locations of retrogradely labeled cells within both MNTB and LNTB imply that they project with non-uniform latencies to cell clusters or perhaps to individual cells in the MSO. Because we were unable to track individual fibers across multiple tissue sections, we measured the Euclidean distance between labeled cells and the injection site as an initial estimate of how cell location can constrain temporal integration of inhibitory inputs. Distance histograms for the two dorsal MSO and one middle MSO injection sites shown in figure 3-3 reveal that MNTB cells were, on average, $800 \mu \mathrm{m}$ closer to the injection site than LNTB cells (Fig. 3-5 A-C; MNTB blue, mLNTB green, pvLNTB red). These differences between MNTB and LNTB occur because the MNTB is situated closer to the MSO than is the LNTB, except at the ventral margin of the MSO, and pvLNTB cells are concentrated in the caudal SOC. Despite being closer to MSO, MNTB cells could be distributed over distances of $1 \mathrm{~mm}$; for the example shown in figure 3-5C, distances ranged from $276-1505 \mu \mathrm{m} ; 874 \pm$ 215 , mean $\pm s d$. This variation in distances within MNTB arises because the MNTB isofrequency laminae extend along the dorsal-ventral extent of the MSO.

We next used the Euclidean distances from labeled neurons to injection sites to estimate IPSP latencies onto MSO cells in a linear summation model. The amount and timing of inhibition delivered from the MNTB, mLNTB and pvLNTB to a cell cluster in the MSO was estimated by convolving each input with an IPSP modeled as an alpha function (see Methods). The conduction velocity of these inputs is not known, so we explored a range from $1.25 \mathrm{~m} / \mathrm{s}$ to $20 \mathrm{~m} / \mathrm{s}$ (rows in Fig. 3-5D) for the case depicted in figure 3-5C. We illustrate this case, because the distances to the injection site from each cell group show the least overlap in the distance histogram and are the most straightforward to interpret. For simplicity, all cells 
in the MNTB and LNTB were activated at the same time, but the relative activation time of MNTB and LNTB was varied (columns in Fig. 3-5D). The composite IPSP of all inputs summed for each cell group and the sum of all cell groups are color-coded (MNTB, green; mLNTB, red; pvLNTB, blue; black, all inputs; Fig. 3-5D).

The IPSP summation procedure for each cell group is illustrated for the MNTB by green dotted lines in figure 3-5D, upper middle panel. Each dotted line is the summed IPSP for all MNTB cells in each $100 \mu \mathrm{m}$ bin of the distance histogram, and thus are partial sums of the entire population of retrogradely labeled MNTB cells. Note that for the slowest conduction velocity $(1.25 \mathrm{~m} / \mathrm{s})$ inputs summed into a population MNTB waveform that was broader than the individual peaks. The pv and mLNTB (red, green traces in top row of Fig. 3-5D) also summed into a unimodal population IPSP broader than individual input waveforms (latter not shown in this figure). Increasing the conduction velocity to $2.5 \mathrm{~m} / \mathrm{s}$ ( $2^{\text {nd }}$ row Fig. 3-6D) smoothed and shortened the duration of the population IPSP to closely resemble the unitary IPSPs for each inhibitory cell group, and the amplitude increased as individual IPSPs became more aligned temporally. At faster conduction velocities this trend of shorter duration and greater amplitude continued for the population IPSP.

The relative time of activation of MNTB and LNTB is unknown, so we explored values that varied up to the maximal ITD of the cat. When MNTB led LNTB activation by $400 \mu \mathrm{s}$ (left column in Fig. 3-5D), a bimodal composite IPSP was conveyed to the MSO for all conduction velocities. At small conduction velocity $(1.25 \mathrm{~m} / \mathrm{s})$ MNTB input leads composite LNTB input by over $1 \mathrm{~ms}$ (1.22 ms; upper right panel in Fig. 3-5D), due to its earlier activation and closer location to MSO. As conduction velocity increases, the distance to MSO becomes less important and the difference in transmission time to MSO $(430 \mu \mathrm{s}$ at $10 \mathrm{~m} / \mathrm{s}$; right column in Fig. 3-5D) tends to the relative time of activation, in this case $400 \mu \mathrm{s}$. When LNTB led or was simultaneous with MNTB activation, differing combinations of ITD and conduction velocity yielded a unimodal or bimodal composite IPSP. For LNTB activation leading MNTB activation by $400 \mu \mathrm{s}$ (left column), due to its longer distance from dorsal MSO injection sites, at slow conduction velocity $(1.25 \mathrm{~m} / \mathrm{s})$ the MNTB input still preceded LNTB input (upper left panel in Fig. 3-5D). A slightly larger conduction velocity $(2.5 \mathrm{~m} / \mathrm{s})$ compensates for the relative distance and both MNTB and LNTB inputs arrive in synchrony at the MSO. At $10 \mathrm{~m} / \mathrm{s}$, the LNTB peak leads the MNTB peak by $280 \mu \mathrm{s}$. At conduction velocity values greater than 20 
$\mathrm{m} / \mathrm{s}$, the LNTB peak would precede the MNTB peak by the relative $400 \mu$ s activation time and compensate for differences in distance to the injection site. Therefore, conduction velocity of inhibitory inputs could affect significantly the relationship between ITD and relative onset of inhibitory inputs to an MSO neuron.

\section{MSO Neuron Model}

Our structural data imply a level of symmetry in bilateral inhibitory circuitry, whereby ipsilateral pvLNTB cells may complement phase-locked contralateral MNTB cell inputs to individual MSO neurons. Cellular models have established basic features of coincidence detectors in the MSO and its homologue in birds, the nucleus laminaris (Brughera et al., 1996; Agmon-Snir et al., 1998; Dasika et al., 2007), and establish a means to explore functional mechanisms for ITD encoding. Therefore, we established a biophysically and structurally realistic MSO cell and explored the consequences of two distinct sources of phase-locked inhibition. In the following paragraphs we report in sequence (1) activation of the model cell by bilateral excitatory inputs (EE); (2) activation by bilateral excitatory inputs and one contralateral inhibitory input (EIE); (3) activation by bilateral excitatory and inhibitory inputs (EIEI). We explored synaptic integration at $250 \mathrm{~Hz}$ to minimize interactions across stimulus cycles and challenge the model to produce the broadest range of best ITD measured experimentally (for review see Joris and Yin, 2007).

EE Model: bilateral excitation, no inhibition. Ten independent excitatory inputs, having characteristics that match published data of spherical bushy cells (see Methods and Fig. 3-1), were applied to each dendrite. Based upon recent suggestion that the number of excitatory inputs per side may be as few as four in gerbils (Couchman et al., 2010), we also modeled the effects of having fewer inputs. Conductances were the same for each input in each run of the model, and a range of conductance values were used across different runs. Afferent firing rate (EPSP rate, or spherical bushy cell rate) was fixed for each input in our model at 150 $\mathrm{sp} / \mathrm{s}$ to simulate a moderate sound level. ITD values were referenced to the contralateral excitatory input, which always occurred at $0 \mathrm{~ms}$.

Using ten inputs per side, a range of conductance values yielded symmetric ITD curves, indicating that the dendritic geometry of the cell did not introduce asymmetries. At low conductance values (5-7nS), few discharges occurred in the troughs of the ITD curves, indicating that action potentials required bilateral coincidence of EPSPs (Fig. 3-6A). At 
greater synaptic strength (10 and $15 \mathrm{nS}$ ), the peak firing rate reflected perfect entrainment (one spike per stimulus cycle) to the $250 \mathrm{~Hz}$ stimulus and began to raise discharge rates in the trough of the ITD curve, indicating unilateral coincidence could generate action potentials. Reducing the number of inputs to 4 per side, while maintaining similar conductance values $(10,12.5,15 \mathrm{nS})$ for a single input, reduced the MSO cell's firing rate relative to ten inputs (Fig. 3-6B). Increasing the conductance of each input toward values measured in vitro from gerbil MSO (22 - 50 nS per input; (Couchman et al., 2010)) led to entrainment across a broad range of ITD values and elevated firing rates in the trough of the ITD curve. When the total conductance was equalized (number of inputs times conductance/input), ten inputs generated narrower ITD curves with sharper peaks than 4 inputs (Fig. 3-6C).

EIE Model: bilateral excitation, unilateral inhibition. We next introduced phase-locked inhibitory inputs into our MSO cell model, which is a configuration employed in most modeling studies of MSO (Brughera et al., 1996; Brand et al., 2002; Dodla, 2005; Zhou et al., 2005; Mathews et al., 2010). Spike trains of inhibitory inputs were assigned similar temporal characteristics (vector strength, firing rate, interspike interval histograms) to excitatory inputs, generally matching a sparse set of published data from low CF MNTB neurons (Tsuchitani, 1994; Smith et al., 1998; Paolini et al., 2001; Kopp-Scheinpflug et al., 2008). Because ten excitatory inputs/side yielded narrower ITD curves than four inputs/side, and four inputs/side was considered a minimum estimate (Couchman et al., 2010), we illustrate model data using ten excitatory inputs/side. Ten independent inhibitory inputs were applied to the soma and treated as originating from the same cell group by having the same average phase value and onset. Inhibitory conductance (10 nS/input) was made greater than excitatory conductance (7 nS/input), in accord with recent measurements in gerbil MSO, but in our initial models we did not utilize the large synaptic conductances that were measured in that study in part because we did not use the small number of inputs that were calculated (Couchman et al., 2010). Individual ITD curves were generated with the average inhibitory delay fixed relative to contralateral excitation. A series of ITD curves was generated as the inhibitory delay varied between runs in $0.1 \mathrm{~ms}$ increments from $-0.5 \mathrm{~ms}$ to $+0.5 \mathrm{~ms}$ (Fig. 3-7 A, D).

Variation of IPSP onset time altered the ITD curve by changing its peak and minimum firing rates, slope, and overall shape. The collection of ITD curves for all inhibitory delays is shown as a 3D plot in figure 3-7A, and all curves are projected onto a 2D surface in figure 3- 
8B. The black curve in figure $3-8 \mathrm{~B}$ is the reference EE curve for the excitatory conductance value (Fig. 3-6A, green trace) of $7 \mathrm{nS} /$ input that was used in these simulations. As has been shown in previous models utilizing phase-locked inhibition, with even lower conductance values per input than are used here (Brand et al., 2002; Zhou et al., 2005), leading inhibition pushed the peak of the ITD curve to positive values. The blue and purple traces in figure 37A highlight this response, as illustrated by dotted lines that drop to the floor of the trace at positive ITD values and within the grey bar, which shows the ecological range of ITD for the cat. IPSP onset values of -0.4 through -0.1 ms re: EPSP onset (see blue through purple family of traces in Fig. 3-7B) shifted the peak of the ITD curve to positive values of +100 or $+200 \mu$ s (asterisks in Fig. 3-8C show peak value for each ITD curve; note expanded scale in Fig. 3-8C to highlight ecological range of ITD). At an IPSP onset time of $0 \mathrm{~ms}$, the peak occurred at $0 \mathrm{~ms}$ ITD. The peak then shifted to negative ITD values of -100 or $-200 \mu$ s with positive IPSP delays (red trace in Fig. 3-7A, red traces in Fig. 3-7B, red asterisk in Fig. 3-7C shows maximal negative peak shift). The peak rate for negative IPSP onset times could exceed those for the corresponding positive IPSP onset times (e.g. - 0.1 compared to +0.1 $\mathrm{ms}$ ), likely due to the asymmetric shape of the individual IPSPs. All inhibitory delays narrowed the ITD curve relative to the EE condition and decreased the overall firing rate, as evidenced by all EIE curves fitting within the boundary of the EE curves generated using the same excitatory conductance (Fig. 3-7B).

EIEI Model: bilateral excitation, bilateral inhibition. A little-explored issue in MSO cellular models is the effect of a second source of inhibition on ITD curves (Leibold, 2010). Given the evidence of an ipsilateral input to MSO from glycine-immunoreactive pvLNTB neurons (Spirou et al., 1998), we modeled this second inhibitory input to be phase-locked with characteristics similar to MNTB inputs. To explore systematically the range of excitatory and inhibitory delay parameters, we applied the additional constraint that inhibitory inputs driven from each ear have a fixed latency relative to their excitatory inputs when generating each ITD curve. Note that this constraint does not obviate the possibility that inhibitory inputs, like some excitatory inputs to cat MSO (Beckius et al., 1999) can operate as delay lines within MSO isofrequency laminae. By exploring a range of relative EPSP/IPSP delays from each side, we explored a distribution of responses that could occur across the MSO cell population. 
For a given pair of fixed IPSP/EPSP relative onset times for each side, the synaptic input from each ear, then, can be appreciated as a composite summed waveform. For clarity of description, we refer to contralateral IPSPs as originating from MNTB and the contralateral composite waveform as the MNTB/cEPSP, and ipsilateral IPSPs as originating from LNTB and the ipsilateral composite waveform as the LNTB/iEPSP. The contralateral composite waveform was fixed in time, and the ipsilateral composite waveform was varied to generate ITD curves. Example composite waveforms are shown when the MNTB and LNTB each lead their excitatory inputs (Fig. 3-8D a - c). The MNTB leads the cEPSP by $-0.3 \mathrm{~ms}$ (green waveform) and the LNTB leads by $-0.2 \mathrm{~ms}$ (blue waveform), and ITD of these waveforms is varied $(-0.2,0,+0.2 \mathrm{~ms}$ shown) to generate the blue ITD curve in figure 3-8D-a. Another pair of composite waveforms depicts MNTB leading and LNTB lagging their excitatory inputs (Fig. 3-8D $d-f$ ). The MNTB leads by -0.3 ms (green waveform identical to Fig. 3-8D a-c) and the LNTB lags by $0.3 \mathrm{~ms}$ (red waveform showing initial positive deflection because iEPSP leads LNTB IPSP). The ITD of these waveforms is varied $(-0.2,0,+0.2 \mathrm{~ms})$ to generate the red ITD curve in panel $A$.

The family of ITD curves in figure 3-8A illustrate the effects of adding the second, LNTB inhibitory input to the MNTB input in the case where the MNTB IPSP has a fixed onset time of -0.3 ms relative to its associated contralateral EPSP. This MNTB onset time led to a shift of the ITD peak in the EIE model to $+200 \mu$ s (Fig. 3-7 A, B), which is plotted as the solid black trace in the 2-D projection in figure 3-8B. For each ITD curve in figure 3-8A, the composite ipsilateral waveform generated by a fixed LNTB/iEPSP onset time difference was shifted in $0.1 \mathrm{~ms}$ increments relative to the composite contralateral waveform. The LNTB/iEPSP onset time difference was then changed, also in $0.1 \mathrm{~ms}$ increments, and the next ITD curve was generated.

When the LNTB led the iEPSP by $-0.2 \mathrm{~ms}$ (Fig. 3-8 A, B; blue bold trace) so that each inhibitory input led its associated excitatory input, the ITD peak was centered at $0 \mathrm{~ms}$. Other composite LNTB/iEPSP waveforms in which the LNTB onset time led the iEPSP $(-0.5$ to -0.1$)$ positioned the peak ITD at values between -100 to $+100 \mu \mathrm{s}$. In effect, they shifted the peak to more positive values than the reference EIE curve, which had a peak ITD at $+200 \mu \mathrm{s}$. However, when the composite LNTB/iEPSP waveform reflected the LNTB lagging the iEPSP by +0.2 to $+0.5 \mathrm{~ms}$, the peak of the ITD curve was at positive values. At a lagging value of 
$+0.3 \mathrm{~ms}$, the peak of the ITD curve was shifted an additional $+100 \mu \mathrm{s}$ relative to the EIE condition (red bold profile in Fig. 3-8 A, B and red asterisk in Fig. 3-8C). Firing rates in figure $3-8 \mathrm{~A}$ are projected onto a topological map in figure 3-8C (same format as in Fig. 3-7), and the peak rate for each ITD curve is plotted as asterisks. Although the second inhibitory input from the LNTB input could further shift the peak of the ITD curve, it reduced firing rates and narrowed ITD curves relative to inhibition from MNTB only, as evidenced by all EIEI ITD curves located inside the corresponding EIE curve (colored traces vs black trace in Fig. 3$8 B)$.

A more complete series of EIEI ITD curves are shown in figure 3-9 for a range of fixed MNTB delays. Each MNTB delay is shown in a separate panel, and the ITD curves for each LNTB/iEPSP onset time difference are plotted in different colors. The color code, shown in the key, is the same as used in figure 3-8, whereby red graphs denote LNTB onset leading its associated EPSP and blue graphs denote LNTB onset lagging its associated EPSP. The corresponding EIE curve for the same MNTB delay, from figure 3-8, is shown as the black trace in each panel. All the other ITD curves in each panel show the effect of adding an ipsilateral inhibitory input and varying the timing of that input relative to ipsilateral excitatory input. The bottom graph in each panel is a 2-D topological projection of rates, as in figures 3$7 \mathrm{C}$ and $3-8 \mathrm{C}$.

In general, when MNTB led and LNTB lagged their respective excitatory input, the ITD curve had a peak at positive ITD values (red traces in Fig. 3-9A, B), as with a single inhibitory input leading contralateral excitation (black traces in Fig. 3-9A, B). When the MNTB onset time led its associated excitatory input by -0.3 (Fig. 3-8), -0.2 (Fig. 3-9A), -0.1 (not shown) or coincident at $0 \mathrm{~ms}$ (Fig. 3-9B), at least one value for LNTB lagging delay shifted the ITD peak an additional $100 \mu$ s more positive in delay (asterisks in bottom row of Fig. 3-9 B, C relative to vertical arrows showing delay for corresponding EIE ITD curve). When both MNTB and LNTB onset times led their associated excitatory input, the ITD curve was centered at or near $0 \mathrm{~ms}$ (blue traces in top row of Fig. 3-9A; asterisks in lower panel).

Conversely, when MNTB onset time lagged and LNTB onset time led their respective excitatory input, the ITD peak was shifted to negative ITD. Recall that when MNTB was the only inhibitory input (EIE model) and it lagged its excitatory input, the ITD peak occurred at negative ITD values (Fig. 3-8). When MNTB onset time lagged its associated excitatory input 
by 0.1 (not shown), 0.2 (Fig. 3-9D) and $0.3 \mathrm{~ms}$ (not shown), at least one value of LNTB onset time leading its excitatory input shifted the ITD peak an additional $100 \mu$ s more negative in delay (asterisks at bottom of Fig. 3-9D). In the final set of timing combinations, when both MNTB and LNTB onset time lagged their respective excitatory input, as when both MNTB and LNTB led their respective excitatory input, the ITD curve peak was located at or near 0 $\mu$ s ITD. Therefore, three results of adding a second inhibitory input are that further small shifts in the peak discharge rate can occur, that the firing rate is decreased relative to the EIE configuration, and that the ITD curves are narrower, as evidenced by all curves located inside of their corresponding EIE curve in figures 3-8 and 3-9. A qualitative assessment, and a consequence of narrowed ITD curves, is that peaks of the ITD curves are sharper relative to a single inhibitory input (Figs. 3-8, 3-9). A general summary is that bilateral inhibition offers a large parameter space for interactions that manipulate the shape and position of the peak of the ITD curve at both positive and negative delays.

Adding an inhibitory input (EIE vs. EE and EIEI vs. EIE) never generated activity outside of the reference ITD curve (blue and red traces relative to black traces in Figs. 3-7 through 3-9). Therefore, EE curves set the confines for peak shifting due to added inhibition, so we investigated the ability to generate larger peak shifts in the context of broader EE curves, which were generated by increasing the conductance of each excitatory input (Fig. 310 , green traces). Panel $3-10 \mathrm{C}$ is a replication of panel $3-9 \mathrm{~B}$ to compare with conditions of greater excitatory conductance. When the excitatory conductance was increased from 7 to 10 nS/input, even doubling the bilateral inhibitory conductance (10 to $20 \mathrm{nS} / \mathrm{input}$ ) yielded broader ITD curves with higher peak rates (Fig. 3-10B). The ITD peak shifted to $+0.3 \mathrm{~ms}$ for more of the ITD curves (LNTB +0.2 to $+0.5 \mathrm{~ms}$ onset time relative to its associated EPSP vs LNTB onset time only at +0.4 ms delay). As a result, the slope of the ITD curve was steep throughout most of the ecological range for more combinations of LNTB onset time. We next investigated large excitatory conductances ( $25 \mathrm{nS} /$ input) and fewer excitatory inputs (4 inputs; Fig. 3-10A), although still at the lower range of conductance values measured in gerbil MSO (Couchman et al., 2010). Under these conditions, the EE ITD curve is very broad, and bilateral inhibition shifts the ITD peak to $+0.5 \mathrm{~ms}$, the largest value in any of our simulations (Fig. 3-10A). This value occurred for LNTB onset time of $+0.5 \mathrm{~ms}$ relative to its associated EPSP. The ITD curve with bilateral inhibition is narrower than with a single inhibitory cell 
group. The peak of the curve is narrower with increased inhibition, but still relatively broad compared with a larger number, but lower conductance inputs. The portion of the ITD curve

within the ecological range is steeper than with a single inhibitory input. These data show that inhibition can shift the ITD peak to larger values and maintain or increase the slope of the steepest part of the ITD curve if there is a larger excitatory background within which to sculpt an activity profile.

Sharper peaks of ITD curves afford the ability to encode sound location using both peak rate and slope coding mechanisms, depending upon the ITD value at which the peak rate occurs. To quantify the effects of inhibition on the utility of these two encoding strategies, we considered locations directly in front of the head, where angular sensitivity to changes in sound location are greatest. We compared slopes of ITD curves between -0.1 to $+0.1 \mathrm{~ms}$ for EIE and EIEI models that had the same MNTB delay, using both 10-input, low and high conductance models depicted in figure 3-10 A, B. First, we consider the low conductance parameters used in the simulations shown in figure 3-7. The slope of each ITD curve through the middle of the ecological range is greatest when the peak is shifted to positive or negative values, because when the peak value is at zero, the slopes on either side of the peak cancel yielding a slope value near 0 , as occurred in the EIE model for MNTB onset at $+0.1 \mathrm{~ms}$ (Fig.3-11A). For each MNTB delay value, at least one LNTB inhibitory delay steepened the slope of the ITD curve (Fig. 3-11). When MNTB onset led contralateral excitation, LNTB onset lagging ipsilateral excitation resulted in more positive slope values. When MNTB onset lagged contralateral excitation, LNTB onset leading ipsilateral excitation resulted in more negative slope values. These observations are illustrated by the EIEI curve crossing through the EIE curve in figure 3-11A. The same general pattern occurred for the high conductance, 4 input model (Fig. 3-11B).

\section{DISCUSSION}

In this study we showed by tract tracing that both major subnuclei of LNTB ( $p v$ and $\mathrm{mLNTB}$ ) innervate the MSO, by stereological counts that relatively few MNTB neurons innervate low frequency MSO and by computational modeling that a second source of inhibitory input can sharpen and shift ITD curves along the temporal delay axis. Euclidean 
distances from labeled LNTB and MNTB cells to dorsal MSO differ greatly, and could contribute distinct phase-locked inhibitory group delays to the binaural processing circuit. Local Inhibitory Neurons in the ITD Circuit: Identity, Numbers and Location

The MNTB and LNTB have been known for at least two decades to be the primary sources of inhibitory inputs to the MSO (Cant, 1991; Cant and Hyson, 1992; Kuwabara and Zook, 1992; Smith et al., 1998). Projections from MNTB arise primarily, if not entirely, from principal neurons, which constitute about $95 \%$ of all neurons in cat (Spangler et al., 1985; Smith et al., 1998). The LNTB is a heterogeneous group of neurons (Warr, 1972; Adams, 1983). By tract tracing, we extend previous descriptions of LNTB input to the MSO (Cant and Casseday, 1986; Cant and Hyson, 1992) showing that both major subdivisions of LNTB, the pvLNTB and mLNTB (Spirou and Berrebi, 1996), project to MSO. One concern with retrograde tract tracer methodology is that unintentional labeling of fibers of passage can mark neurons that do not project to the injection site. Notably, large tract tracer injections surrounding the dorsal, medial and ventromedial MSO over large rostro-caudal distances did not label more than an occasional pvLNTB cell. In contrast, tens of pvLNTB neurons were labeled from small injections into MSO. We were unsuccessful in confining small placements of anterograde tracer to the odd geometry of pvLNTB. Even with success, interpretation of data would be compromised because many SBC afferent fibers to MSO pass directly through the pvLNTB and could be labeled at the injection site. By omitting from consideration injection sites that extended outside the cellular borders of the nucleus, we are confident that we are mapping projections to particular loci within MSO. It is noteworthy that due to the restricted size of our injection sites, we were able to describe tonotopic features of MNTB projections to low frequency MSO regions.

Several authors have noted the sparse branching of individual MNTB axons within MSO and contact of branch terminals with multiple MSO somata via a few, small inputs per cell body (Kuwabara and Zook, 1992; Smith et al., 1998; Werthat et al., 2008; Couchman et al., 2010). In our study, the smallest BDA injection sites labeled between $11-37$ MNTB neurons. These observations, together with our counts of a 1:2 ratio of MNTB to MSO cells at $\mathrm{CF}<5 \mathrm{kHz}$, are consistent with physiological estimates of small numbers of convergent MNTB inputs (lower bound estimate of $2-3$ ) to a single MSO cell in brain slices (Couchman et al., 2010). In vivo labeling shows innervation of widely separated rostro-caudal locations by 
a single axon, which is not captured in vitro, so the use of 10 inputs/side in biophysical models (Zhou et al., 2005; this study) is a reasonable estimate. To achieve this innervation ratio, each low frequency MNTB cell would need to innervate 20 low frequency MSO cells, but note that at CF $>5 \mathrm{kHz}$, where the cell counts reveal a 3 MNTB: 1 MSO cell ratio, each high frequency MNTB cell would need to innervate only 3-4 MSO cells. Inspection of single axon reconstructions using light microscopy, which are a mix of low and high frequency MNTB cells, is consistent with this range in the number of postsynaptic targets (Kuwabara and Zook, 1992; Smith et al., 1998; Werthat et al., 2008; Couchman et al., 2010). In general support of these numbers, electron microscopic studies estimate a significant, roughly even match of inhibitory and excitatory input to the MSO cell body (Clark, 1969b; Brunso-Bechtold et al., 1990; Russell and Moore, 2002).

The bulk of inhibitory input has been projected, using immunocytochemistry, to originate from the MNTB in gerbils (Couchman et al., 2010). Across experiments, we labeled more MNTB than LNTB neurons (209 vs 168), and within LNTB more pvLNTB than mLNTB cells (115 vs 53 ). We noted that injections located lateral or medial to the MSO cell column labeled LNTB or MNTB cells preferentially. Based upon structural parallels between mLNTB and VCN stellate neurons, the latter of which can phase-lock well to low frequency sound $(<1$ kHz; Blackburn and Sachs, 1989; Wiegrebe and Meddis, 2004), we modeled all LNTB inputs as phase-locked. Given uncertainties in absolute lateral-medial location of injection sites in the MSO cell column, we used the simplest assumption that MNTB and LNTB contributed equal numbers of inputs to an MSO cell. At higher acoustic frequencies, mLNTB cells could contribute tonic inhibitory input, as has been incorporated into some MSO models (Svirskis et al., 2003; Zhou et al., 2005).

Architectural design of the brain must account for interaction between locations of afferent neurons, their time of activation and their conduction velocity to effect synaptic integration at a postsynaptic site. These temporal constraints are amplified in the MSO circuit, where shifts of tens of microseconds in latency dramatically affect firing rate. We show that inhibitory cell populations can be separated by at least $1 \mathrm{~mm}$ but the effects of this distance, when MNTB leads LNTB activation, are minimized by axonal conduction velocities of at least $10 \mathrm{~m} / \mathrm{s}$. However, because LNTB cells are further from most locations in MSO, faster conduction velocities (> $20 \mathrm{~m} / \mathrm{s}$ ) are required to ensure not only that LNTB inputs arrive first 
at MSO but also that the temporal delay will match the relative time of activation of the LNTB and MNTB cell groups. MNTB axons could compensate by having longer trajectories (see Smith et al., 1998), but these distances would have to be longest for innervation of more rostral locations in dorsal MSO, especially for rostral MNTB cells. Furthermore, the caudal concentration of retrogradely labeled pvLNTB cells suggests a possibility for an ipsilateral inhibitory delay line. Even at conduction velocities of $10 \mathrm{~m} / \mathrm{s}$, delays of at least $250 \mu \mathrm{s}$ could be achieved across the $4 \mathrm{~mm}$ rostro-caudal extent of cat MSO. For a given sound location, then, the temporal relationship between ipsilateral excitation and inhibition may vary among MSO neurons having similar CF. Note that distances from pvLNTB and MNTB are more similar for middle-ventral locations in MSO, and many LNTB neurons are closer than MNTB neurons to ventral locations in MSO. Therefore, timing requirements for interaural processing and the functions of binaural processing may differ in these regions of MSO.

\section{Binaural Models}

The reconstructed cell used in our model had striking bipolar orientation of dendrites, but also incompletely reconstructed rostro-caudal dendrites, as has been reported for cat MSO (Scheibel and Scheibel, 1974; Lindsey, 1975; Schwartz, 1977; ferret, Brunso-Bechtold et al., 1990) which were removed to simplify the computation and its interpretation. The cell had an axon emanating from the soma, and biophysical properties that incorporated recent information about the locations and relative densities of $\mathrm{K}_{\mathrm{LT}}$ and $\mathrm{Na}$ channels in gerbils (Matthews et al., 2010; Scott et al., 2010). Given anatomical information about excitatory innervation of MSO, we explored a range from four, based upon minimal estimates in brain slices (Couchman et al., 2010), to ten inputs per side, in line with previous MSO models (Zhou et al., 2005). The smaller number of excitatory inputs/side with larger conductance/input yielded broader and shallower ITD curves in the absence of inhibition. These parameters resulted in greater monaural activity, evidenced by significant activity in the trough of the ITD curve. This amount of activity is typically not present in recordings from MSO neurons in the absence of inhibition (Brand et al., 2002; other ms). However, an advantage of a broad excitatory ITD curve is that it provides a larger ITD range in which inhibition can carve out a greater range of shifts in the peak of the ITD curve.

The demonstration that pvLNTB cells innervate MSO, and consideration that mLNTB cells, like VCN chopper cells, phase lock to low acoustic frequency underlie exploration of a 
second source of phase-locked, ipsilaterally driven inhibition, attributed to the LNTB, on ITD coding of low frequency stimuli. One effect was to shift the peak ITD by an additional 100-200 $\mu$ s relative to a similar shift effected in EIE models (Brand et al., 2002; Zhou et al., 2005; this study), but only if the LNTB input lagged the ipsilateral excitation. Indeed, LNTB inhibition leading ipsilateral excitation could shift the ITD peak to $0 \mathrm{~ms}$, as has been achieved through increased static conductance of MSO neurons in models and application of strychnine in vivo (Pecka et al., 2008b), and even to small, negative ITDs, which have been recorded in vivo. Despite this additional shift, we were unable to shift the peak ITD to values near $1 \mathrm{~ms}$, which have been reported for some low CF MSO cells (Yin and Chan, 1991). However, if this condition were riding upon neural delays, which could achieve another 300-400 $\mu$ s in cat based upon axonal measurements and conduction velocity estimates (Beckius et al., 1999; Karino et al., 2011), then these combined mechanisms could generate shifts approaching 1 ms. Additional mechanisms, such as asymmetric excitatory inputs (Jercog et al., 2010), emergence of the axon from a dendrite rather than the cell body (Zhou et al., 2005), and mismatched tuning of excitatory inputs, could further accumulate to achieve large peak shifts. Unfortunately, few data exist for all structural elements of this binaural circuit, especially for BFs less than $500 \mathrm{~Hz}$. In gerbils, most neurons treated with strychnine shift their ITD peak to or near $0 \mathrm{~ms}$, arguing against the aforementioned mechanisms. However, this database is confined to a species that has a small ecological range for ITD.

A clear effect of bilateral inhibition is to suppress firing rate well-below values achieved without inhibition. Slopes are steepened through the ecological range and can decline to zero rate, which could improve localization by single neurons due to improved signal relative to background activity. The slope extending away from the ecological range is also steepened, with the resultant effect of sharpening the peak of the curve. A sharper peak affords greater tolerance to variation in location of the peak near or outside the ecological range, whereas a rounded peak would offer little coding value if it extended into the ecological range. A sharper peak also has value in coding strategies that identify the peak rate, including a Jeffress type model where best ITD is mapped onto the MSO. In a general sense, inhibition carves activity out of the EE curve. Therefore, small numbers of high conductance excitatory inputs or larger numbers of moderate conductance excitatory inputs generate broad ITD curves and provide the template for large shifts in peak ITD. Application of strychnine in vivo does generate some 
cells having broad ITD curves with significant activity in the trough of the curve. Therefore, some MSO cells may be innervated in this manner to achieve the recorded population distribution of peak ITD. Bilateral inhibition may afford the ITD sensitive binaural circuitry a flexible mechanism, layered on top of other cellular mechanisms, to tune the ITD curve and offer animals with larger heads multiple mechanisms to encode ITD within the same cell group. 


\section{Appendix}

\section{Sodium current}

$$
\begin{aligned}
I_{N a} & =\bar{g}_{N a} \cdot m^{4}(0.993 \cdot h+0.007) \cdot\left(V-V_{N a}\right) \\
m_{\infty} & =[1+\exp (-(v+46) / 11)]^{-1} \\
h_{\infty} & =[1+\exp ((v+62.5) / 7.77)]^{-1} \\
\tau_{m} & =\left(-0.826 \cdot[1+\exp ((-20.5-V) / 10.8)]^{-1}+0.141\right) \cdot T f^{-1} \\
\tau_{h} & =\left(-3.74 \cdot[1+\exp ((-40.6-V) / 5.05)]^{-1}+4\right) \cdot T f^{-1} \\
T f & =3^{(T-22) / 10}
\end{aligned}
$$

\section{Low-threshold $\mathrm{K}$ current}

$$
\begin{aligned}
& I_{L T}=\bar{g}_{L T} \cdot w^{4} \cdot(0.82 \cdot z+0.18) \cdot\left(V-V_{K}\right) \\
& w_{\infty}=1 \cdot[1+\exp ((V+57.3) /-11.7)]^{-1} \\
& z_{\infty}=1 \cdot[1+\exp ((V+57) / 15.44)]^{-1} \\
& \tau_{w}=-0.382+1.29 \exp (-(V+70) / 8.82)+0.876 \exp (-(V+70) / 61.9) \\
& \tau_{z}=41.9-32.2 \cdot[1+\exp (-(V+55.4) / 9.85)]^{-1}
\end{aligned}
$$

High-threshold $\mathrm{K}$ current

$$
\begin{aligned}
& I_{H T}=g_{H T}\left[\phi n^{2}+(1-\phi) p\right] \cdot\left(V-V_{K}\right),(\phi=.85) \\
& n_{\infty}=[1+\exp (-(V+15) / 5)]^{-1 / 2} \\
& p_{\infty}=[1+\exp (-(V+23) / 6)]^{-1} \\
& \tau_{n}=100 \cdot[11 \exp ((V+60) / 24)+21 \exp (-(V+60) / 23)]^{-1}+0.7 \\
& \tau_{p}=100 \cdot[4 \exp ((V+60) / 32)+5 \exp (-(V+60) / 22)]^{-1}+5
\end{aligned}
$$

Hyperpolarization-activated cation current

$$
\begin{aligned}
I_{h} & =\bar{g}_{h} \cdot r \cdot\left(V-V_{h}\right) \\
r_{\infty} & =[1+\exp ((V+76) / 7)]^{-1} \\
\tau_{r} & =\left(10^{5} \cdot[237 \exp ((V+60) / 12)+17 \exp (-(V+60) / 14)]^{-1}+25\right) / \text { tadj }
\end{aligned}
$$


Leak current

$$
I_{l k}=\bar{g}_{l k} \cdot\left(V-V_{l k}\right)
$$




\begin{tabular}{cccc}
\multicolumn{5}{l}{ Tonotopy of Neuron Counts } \\
\hline Cell Group & $<\mathbf{2 ~ k H z}$ & $\mathbf{2 - 5} \mathbf{~ k H z}$ & $>\mathbf{5 ~ k H z}$ \\
\hline MSO & $39 \pm 2 \%(3030)$ & $34 \pm 1 \%(2671)$ & $27 \pm 1 \%(2130)$ \\
MNTB & $17 \pm 2 \%(1568)$ & $13 \pm 1 \%(1246)$ & $70 \pm 3 \%(6564)$ \\
\hline Ratio MNTB/MSO & .52 & .47 & 3.08
\end{tabular}

Table 3-1.

Cell counts in MSO and MNTB were assigned to tonotopic regions based on Guinan et al (1972). 


\section{Labeled Cell Populations}

\begin{tabular}{|c|c|c|c|c|c|}
\hline site & location & MNTB & mLNTB & pvLNTB & totals \\
\hline 1 & $D-R$ & 11 & 1 & 1 & 13 \\
\hline 2 & $D-M$ & 37 & 7 & 6 & 50 \\
\hline 3 & $D-M$ & 14 & 4 & 3 & 21 \\
\hline 4 & $D-C$ & 111 & 33 & 55 & 199 \\
\hline \multirow[t]{2}{*}{6} & $M-M$ & 54 & 4 & 50 & 108 \\
\hline & totals & 227 & 49 & 115 & 391 \\
\hline
\end{tabular}

Table 3-2.

Number of cells labeled retrogradely via BDA injections in the MSO. 
A

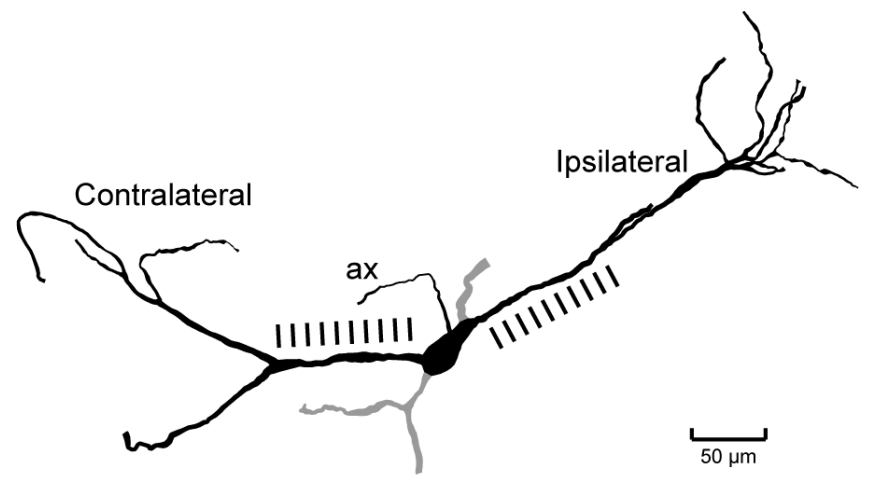

B

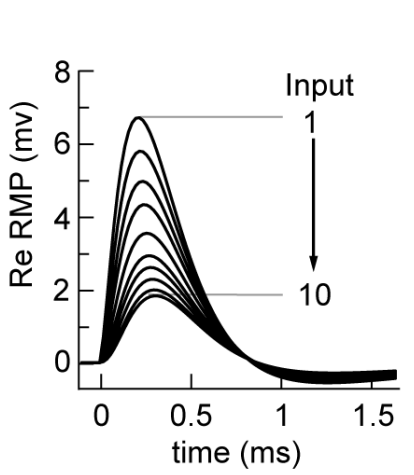

C

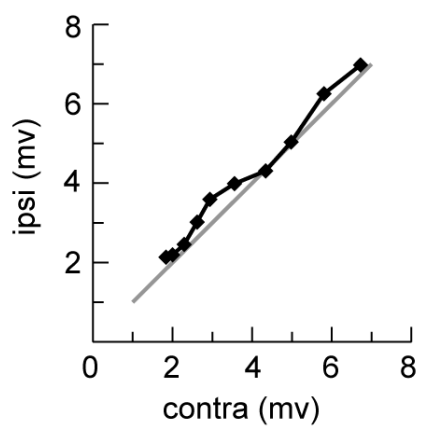

D

$250 \mathrm{~Hz} @ 150$ sp/s

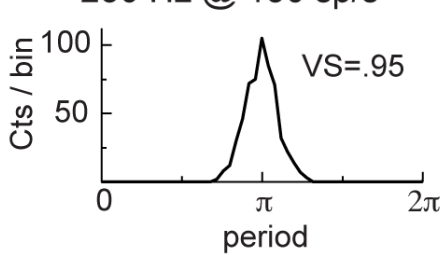

E

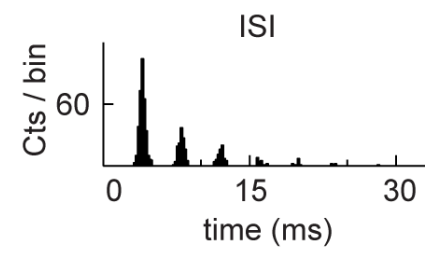

Figure 3-1.

Model MSO Neuron. A, MSO neuron reconstructed and imported into NEURON. Standard model had ten excitatory inputs varied in location along each proximal dendrites (black tics) and twenty inhibitory inputs distributed equally on the soma, ten ipsilaterally driven and 10 contralaterally driven. The reduced input model had 4 excitatory inputs distributed along each proximal dendrite and 8 inhibitory inputs on the soma, again 4 ipsilaterally and 4 contralaterally driven. Dendrites shown in grey were incomplete, and removed from our model. B, Inputs proximal to cell body showed greatest effect for RMP depolarization. C The contralateral and ipsilateral EPSP amplitudes were approximately symmetric at each location. D, Input event times for EPSPs and IPSPs followed a Gaussian distribution and had vector strengths (VS) within the biological range observed in spherical bushy cells. E, Interspike intervals of the phase-locked event times shown in D. Scale bar $=50 \mu \mathrm{m}$ 

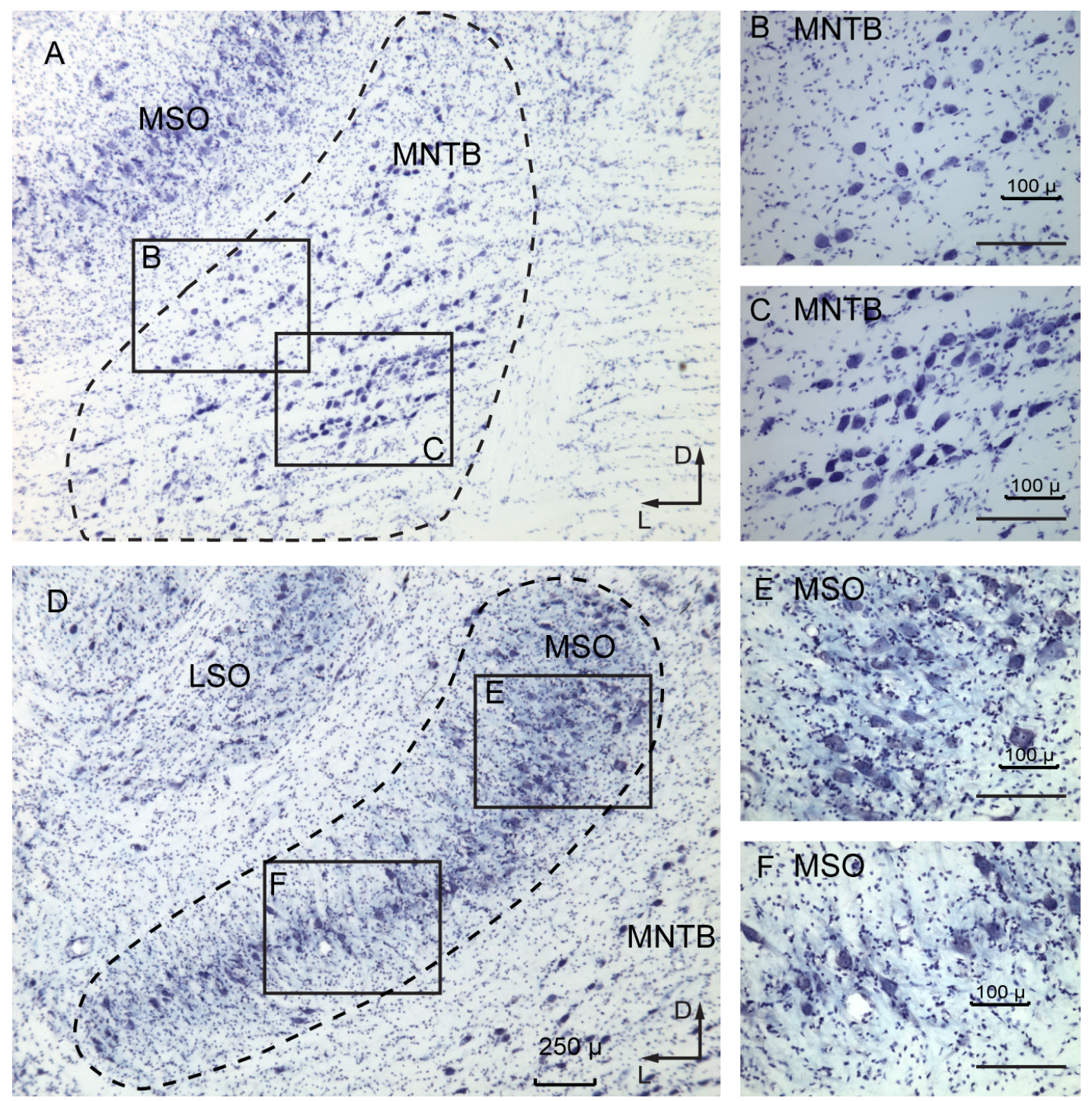

Figure 3-2.

Cell density varies across tonotopic location in MNTB and MSO. A-C, Cell density is sparser laterally in the low frequency region $(B)$ of the MNTB compared to the high frequency region (C). D-F, The converse applies to the MSO where the dorsal low frequency region (E) is multiple cell layers wide and cells in the mid $(F)$ and high frequency regions are stacked, 1-2 cells wide. A, D Scale bars $=250 \mu \mathrm{m} . \mathrm{B}, \mathrm{C}, \mathrm{E}, \mathrm{F}$ Scale bars $=100 \mu \mathrm{m}, \mathrm{L}=$ lateral, D=dorsal. 

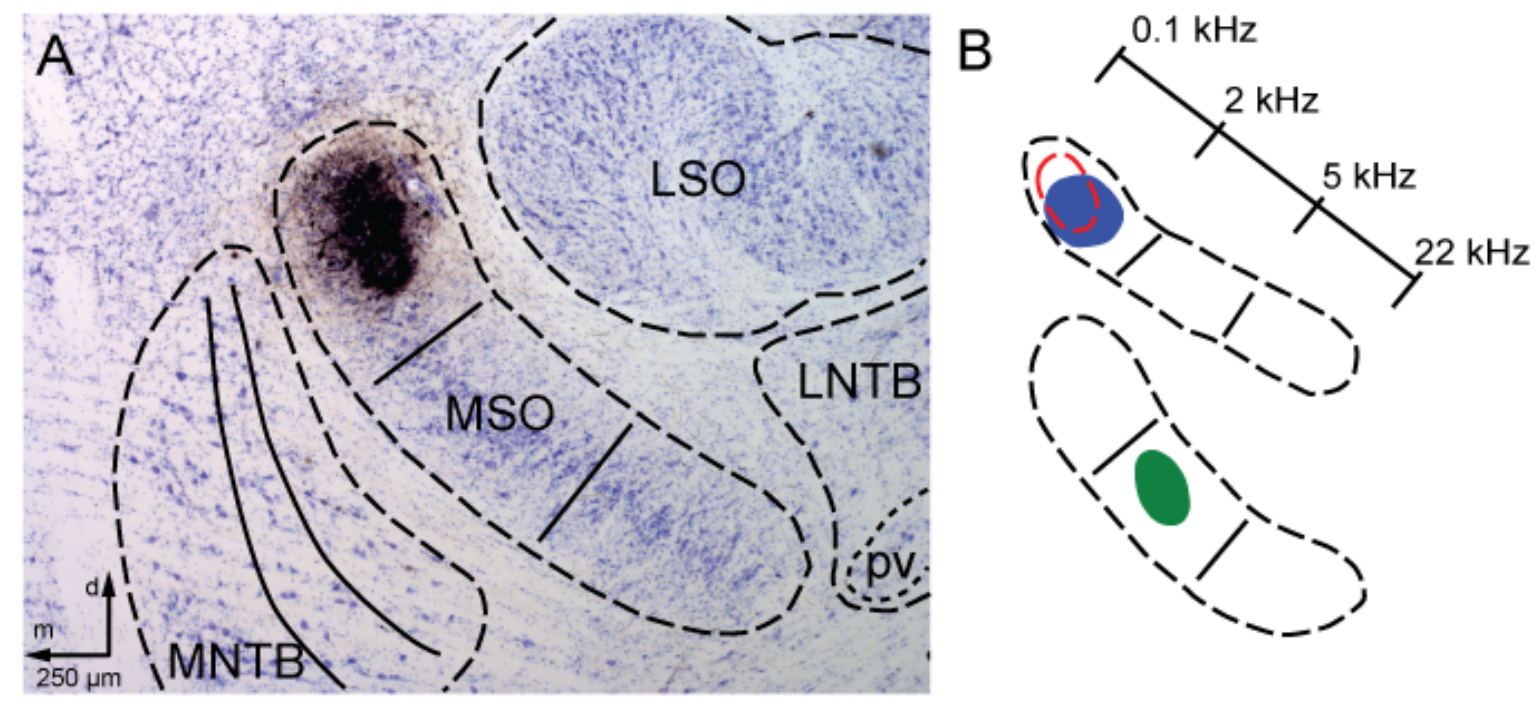

C

D

$\mathrm{E}$

$\mathrm{F}$

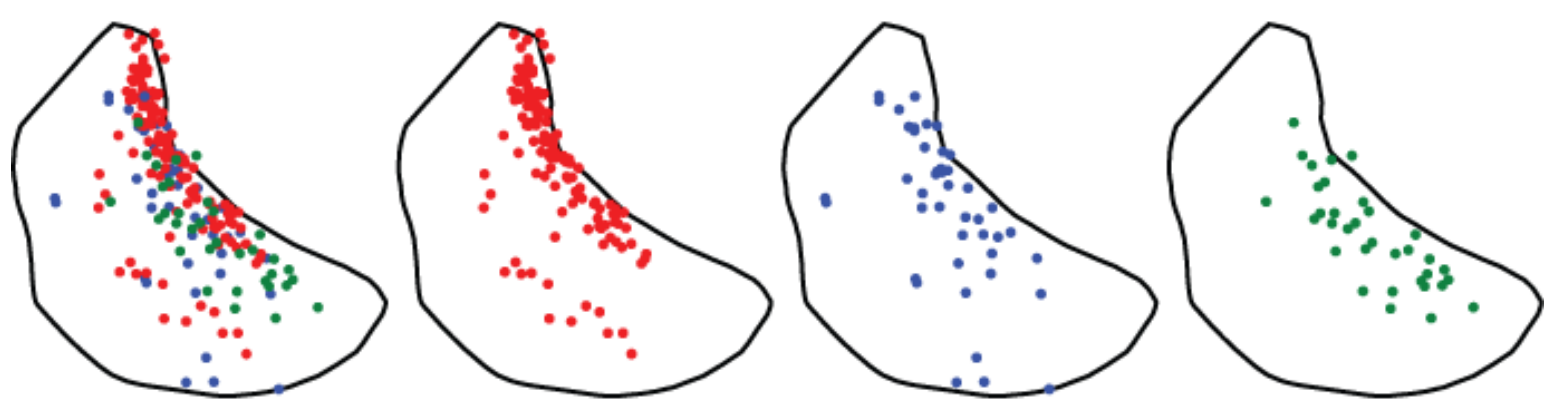

Figure 3-3.

Retrograde labeling of inputs to the MSO. A, Example of a small injection site contained within the borders of the MSO. MNTB, LNTB, MSO and LSO are identified by dashed lines; pvLNTB cells surrounded by a dotted line. Scale bar $=250 \mu \mathrm{m}$. B, Injection sites were located, along the dorsal-ventral axis of the MSO. Solid lines in the MSO and MNTB partition these cell groups into thirds along their tonotopic axes (panels A and B, Guinan et al. 1972). C, Composite of labeled cells (D-F) shows subtle shift in tonotopic location in MNTB at frequencies $<5 \mathrm{kHz}$. D corresponds to the injection site in panel $A$, outlined in red in panel $B$. $E, F$, are shown with color correspondence in B. Scale bar $=250 \mu \mathrm{m}, \mathrm{m}=$ medial, $d=d o r s a l$ 
A

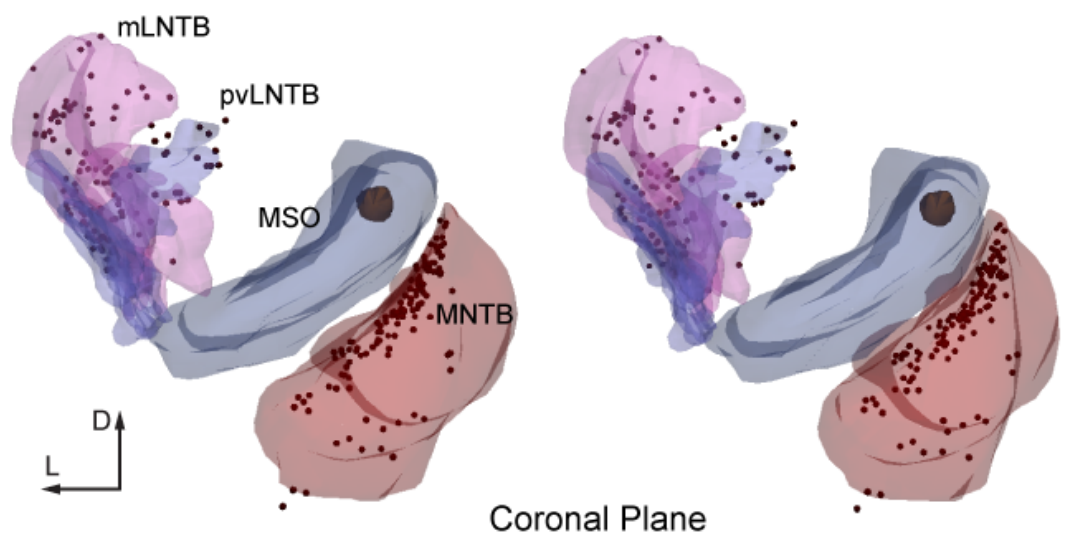

B

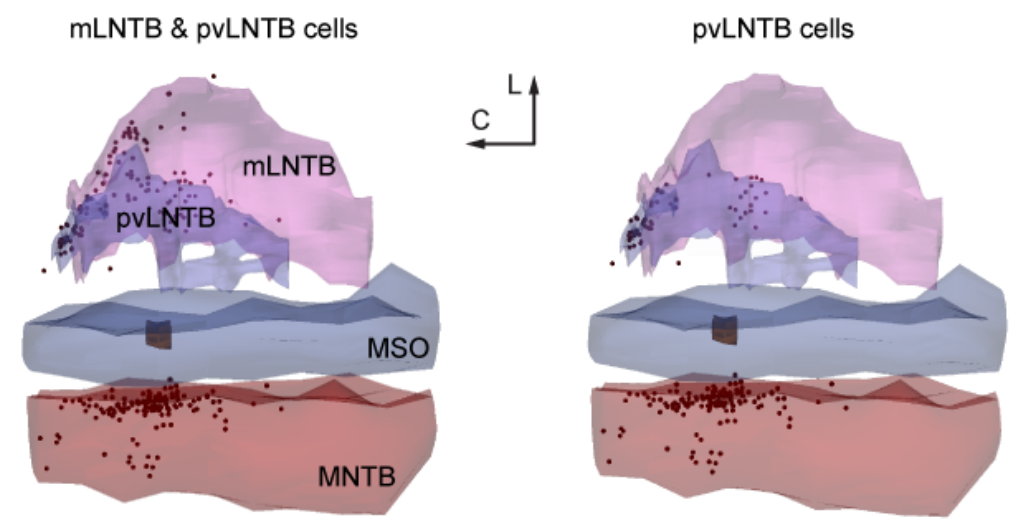

Horizontal Plane

C

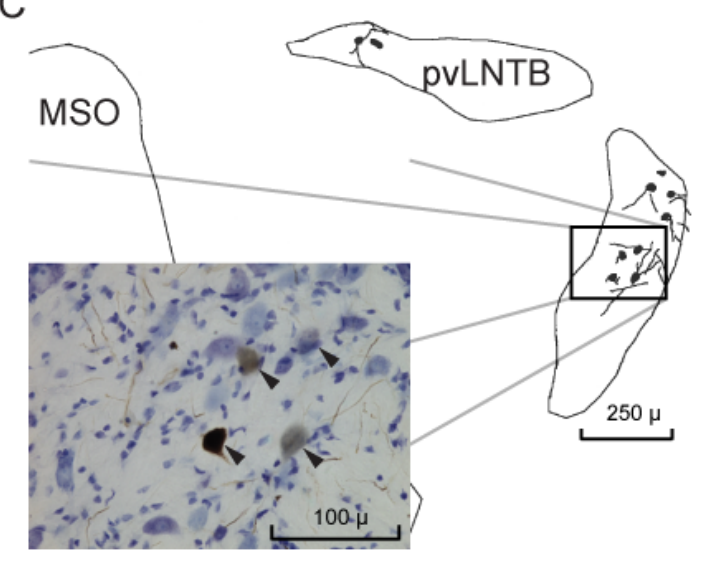

D

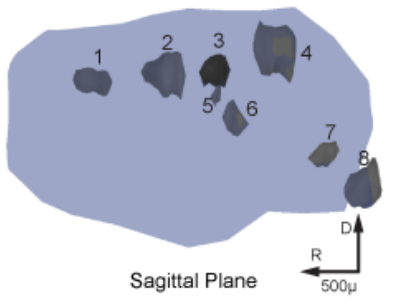

E

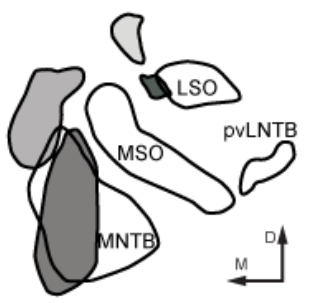

Coronal Plane 
Figure 3-4.

Distribution of retrogradely labeled cells in MNTB and LNTB from focal MSO injection site (shown in Fig 3-3A). A, Stereo pair (coronal view) shows the location of the BDA injection site in the MSO (dark blue) relative to labeled cells in the MNTB (red), pvLNTB (blue) and mLNTB (pink). B, Horizontal view shows that cells labeled in pvLNTB are mostly restricted to the caudal region of the SOC. C, Photographic examples of retrogradely labeled pvLNTB cells (4 labeled cells pictured in inset, arrows). This single section contained 10 labeled pvLNTB cells, as depicted in this camera lucida drawing. pvLNTB split by posterior periolivary cell group ( $\mathrm{pPO}$ ) at caudal locations. $\mathbf{D}$, Injection sites viewed in approximately sagittal plane (side view of MSO) show they were mostly located in dorsal regions. (site 4 shown in Fig. 3-3 A, B, D in red, site 2 in Fig. 3-3 B, E in blue, site 6 in Fig 3-3. B, F in green) E, Across four other cases, injection sites surrounding the MSO labeled only one cell in the pvLNTB and few in the mLNTB. A, B, D, E, scale bar $=500 \mu \mathrm{m}$. C, scale bar $=250 \mu \mathrm{m}$ and inset scale bar $=$ $100 \mu \mathrm{m}, \mathrm{R}=$ rostral, $\mathrm{D}=$ dorsal, $\mathrm{M}=$ medial, $\mathrm{L}=$ lateral, $\mathrm{C}=$ caudal. 

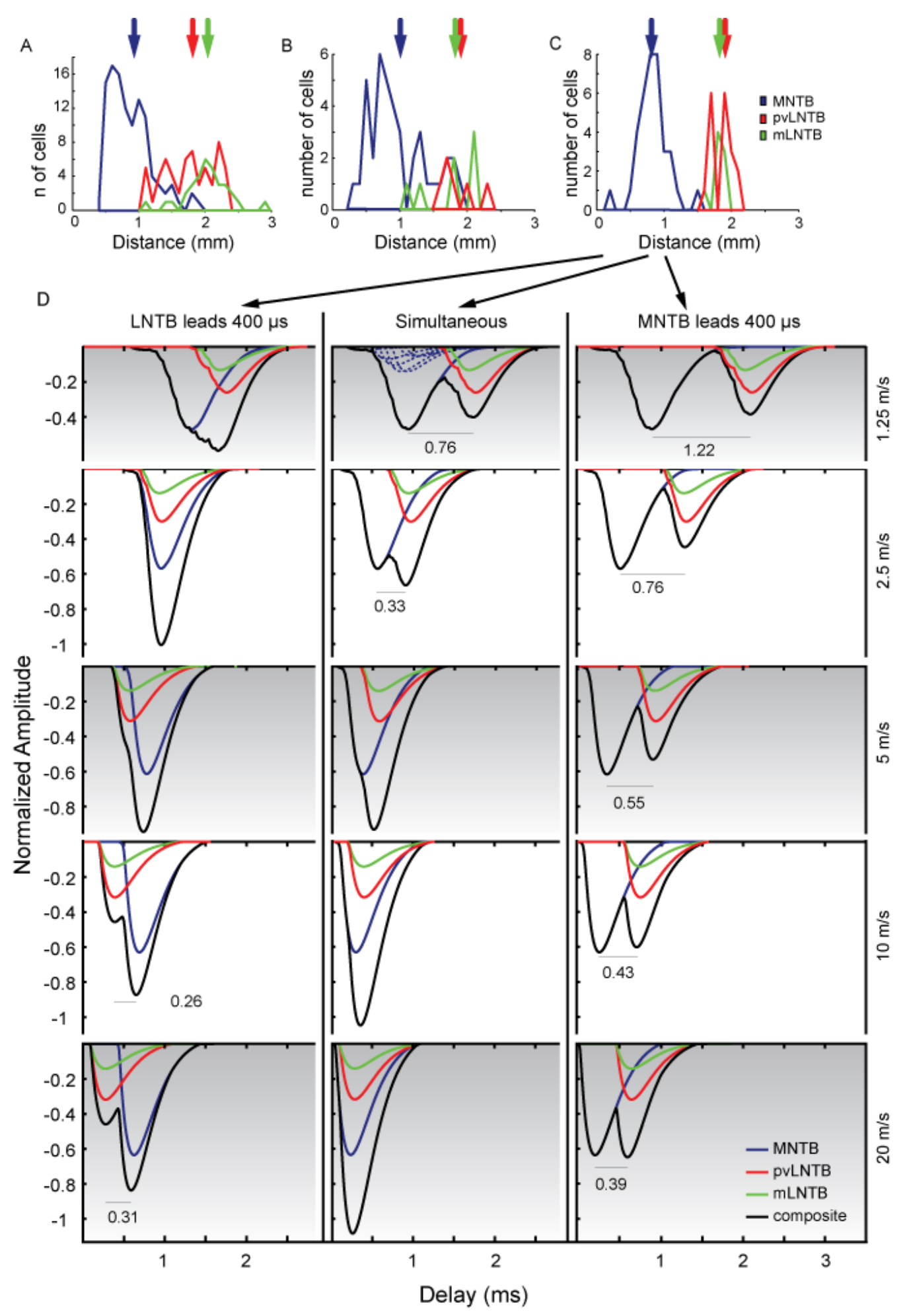
Figure 3-5.

Composite IPSPs from inhibitory cell groups based on location and axonal conduction velocity. A-C, Histograms of Euclidian distances from BDA injection sites for 3 experiments. Distances were measured and binned (100 $\mu \mathrm{m}$ bins) from the injection site to labeled MNTB (blue), pvLNTB (red) and mLNTB (green) cells. D, To generate composite IPSPs, the cell distances (in C) were convolved with an IPSP. We then varied the axonal conduction velocities (rows) as well as the relative MNTB-LNTB latency to activation (MNTB, blue; pvLNTB, red; mLNTB, green; composite IPSP, black). 
A

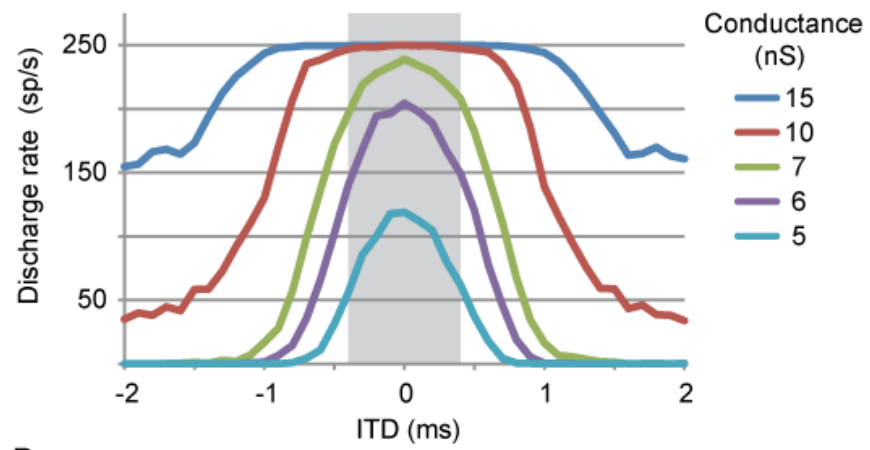

B

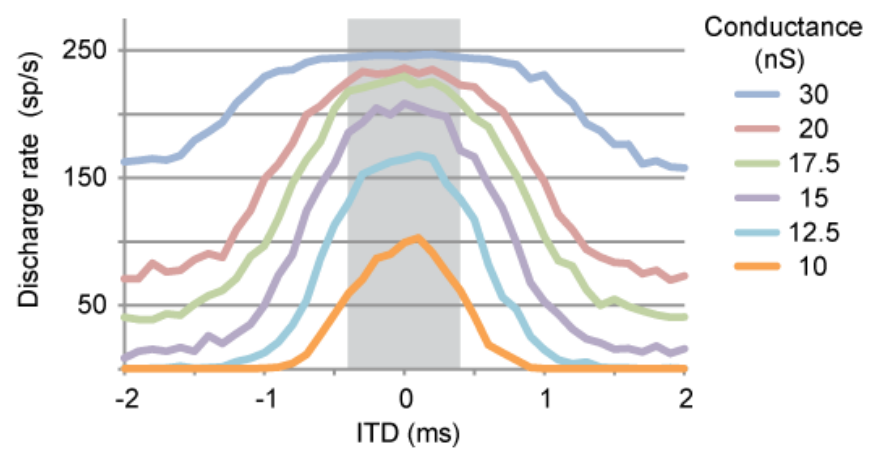

C

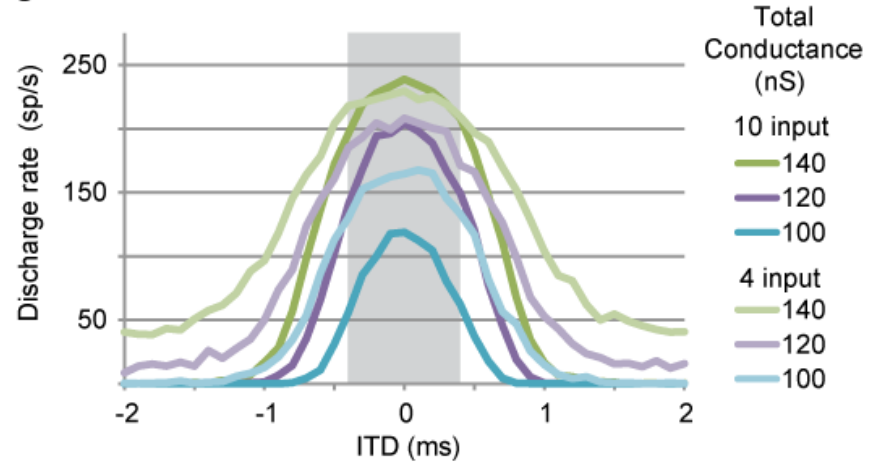

Figure 3-6.

ITD curves generated with bilateral excitatory inputs - no inhibition (EE model). Contralateral EPSPs were fixed at 0 ITD while ipsilateral EPSPs were varied from $-2 \mathrm{~ms}$ (leading) to $+2 \mathrm{~ms}$ (lagging) for $250 \mathrm{~Hz}$ stimulus frequencies. Discharge rate for individual inputs (either 10 or 4 per side) was fixed to $150 \mathrm{sp} / \mathrm{s}$. A. Ten inputs per side. Conductance values $>7 \mathrm{nS} / \mathrm{input}$ led to entrainment of model cell to $250 \mathrm{~Hz}$ stimulus frequency and elevated rate in trough of ITD curve. B. Four inputs per side. At a conductance (30 nS) yielding a saturated rate (250 s/s), a higher trough rate is generated in comparison to 10 inputs (panel A). C. When total input conductance (\# of inputs X conductance/input) is identical, 10 inputs generate narrower ITD curves. 


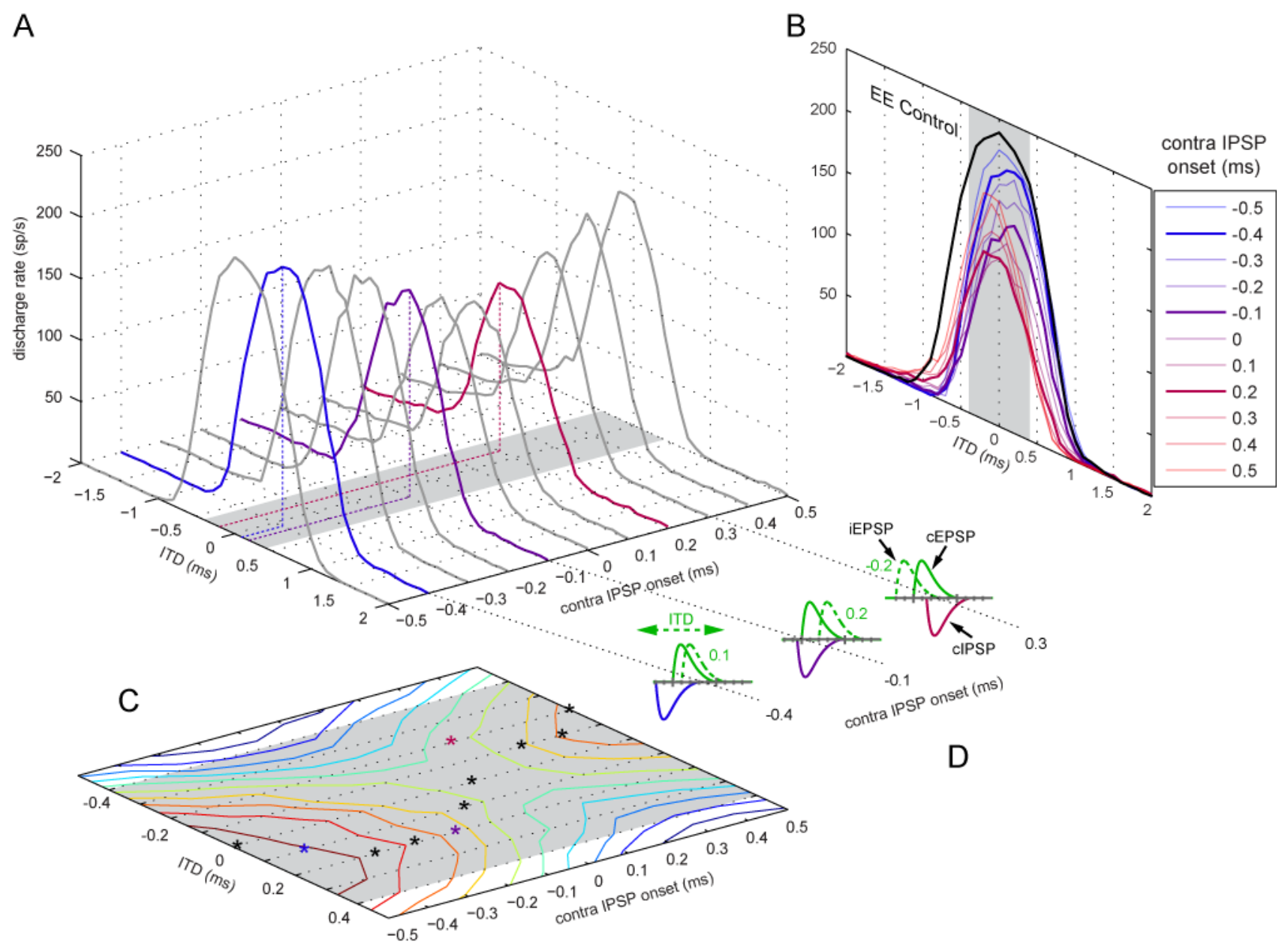

Figure 3-7.

ITD curves generated with a bilateral excitatory, unilateral inhibitory (EIE) model. A. Family of ITD curves plotted against IPSP delay (IPSP onset), measured relative to contralateral EPSP, to generate a 3-D graph. Leading IPSPs shift ITD peak to positive delays. Blue and purple traces illustrate shifts of 100 and $200 \mu s$ (maximal positive shift) of ITD peak. Lagging IPSPs onset time shifted ITD peak to negative delays with maximum shift of -200 $\mu$ s (red trace). B. Projection of traces in A onto a single surface. C. Topologic projection of panel A; note restricted range of ITD values shown. Asterisks indicate peak rate of ITD curve and shift from positive to negative ITD values as inhibitory delay shifts from negative to positive values. Physiological range of ITD for cat indicated by gray band. D, A schematized view of temporal interactions between bilateral excitatory post-synaptic potentials (EPSPs). cEPSP (solid green) fixed at $0 \mathrm{~ms}$ ITD, iEPSP (dashed green) varied over $\pm 2 \mathrm{~ms}$, shown for three fixed values of IPSP delay (blue, purple, red) corresponding to colored traces in panel A. 
A

B

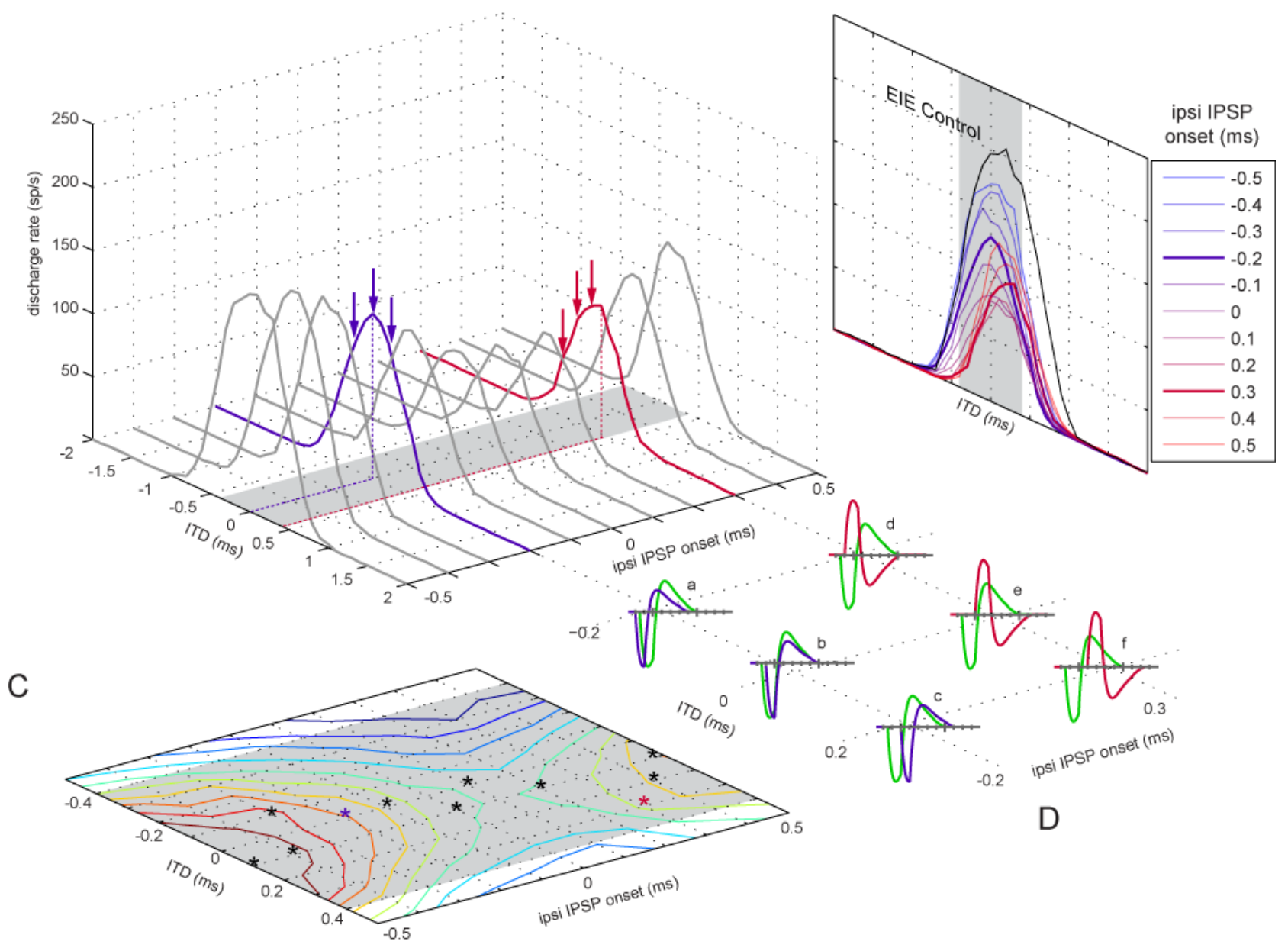


Figure 3-8.

ITD curves generated with a bilateral excitatory, bilateral inhibitory (EIEI) model are narrower than with a unilateral inhibitory input. A. For these simulations the contralateral inhibition was fixed $(-0.3 \mathrm{~ms})$ relative to the contralateral excitation generating a bipolar composite IPSP/EPSP (green trace in panel D). Ipsilateral inhibition fixed relative to the ipsilateral excitation for each ITD curve, but varied \pm 2 ms across ITD curves (LNTB inh onset). B 2-D projection of family of ITD curves in panel A. Peak discharge rates occurred near 0 ITD when both inhibitory inputs lead excitation (purple ITD curve in panel A; blue and purple curves in panel B). Peak discharge rate shifted to positive ITD values for lagging LNTB onset (red and some purple ITD curves). C, Topological projection of panel A. Asterisks indicate peak discharge rate. Physiological range of ITD for cat indicated by gray band. D. For two values of LNTB inhibitory onset (a-c, $-0.2 \mathrm{~ms}$; d-f, $0.3 \mathrm{~ms}$ ), shift of composite ipsilateral waveform relative to composite contralateral waveform (contralateral IPSP-EPSP delay $-0.3 \mathrm{~ms}$, green traces in a-f) is shown. Contralateral EPSP onset fixed to $0 \mathrm{~ms}$. 
A
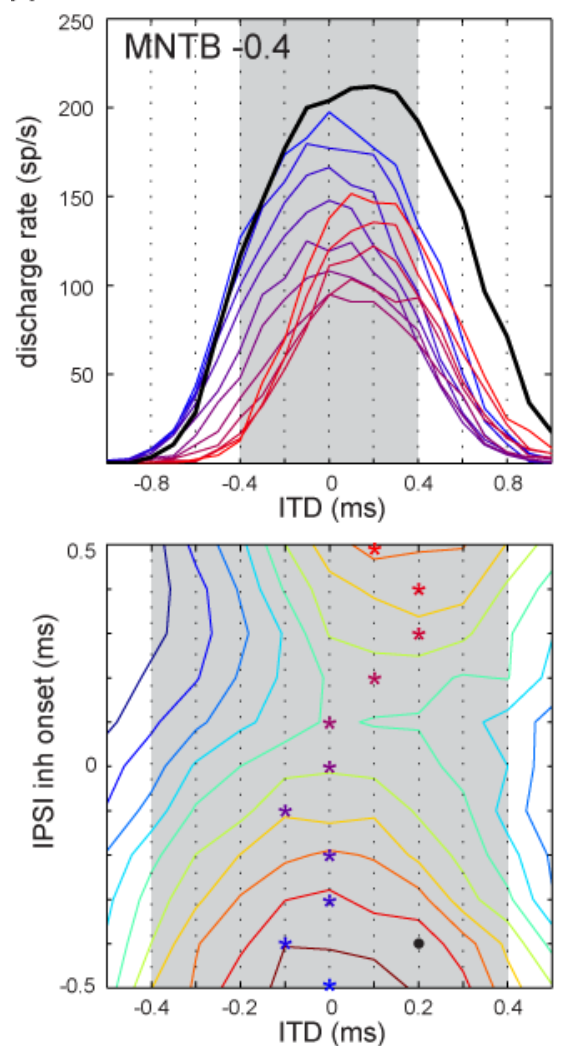

D
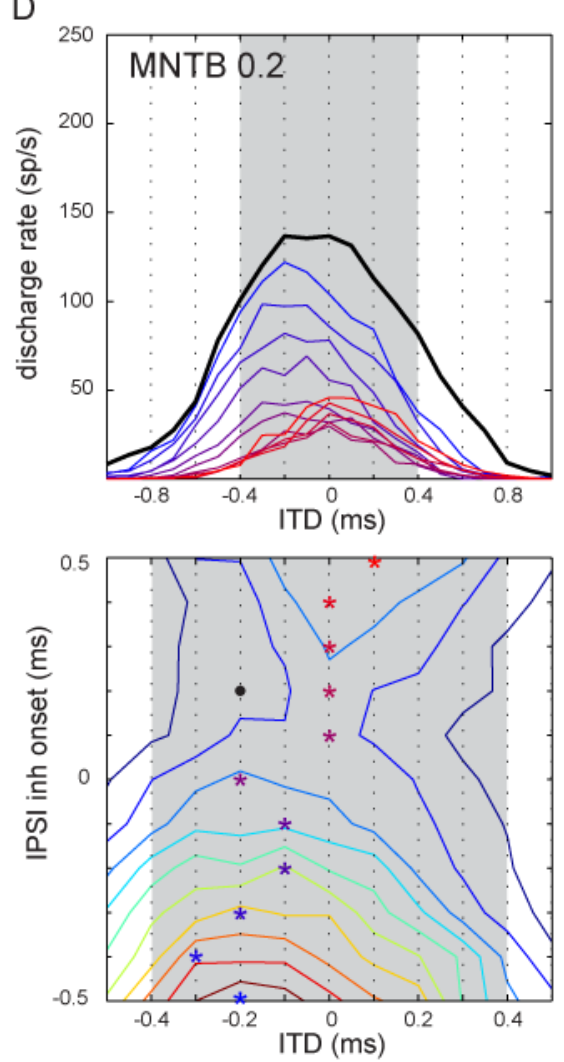

B
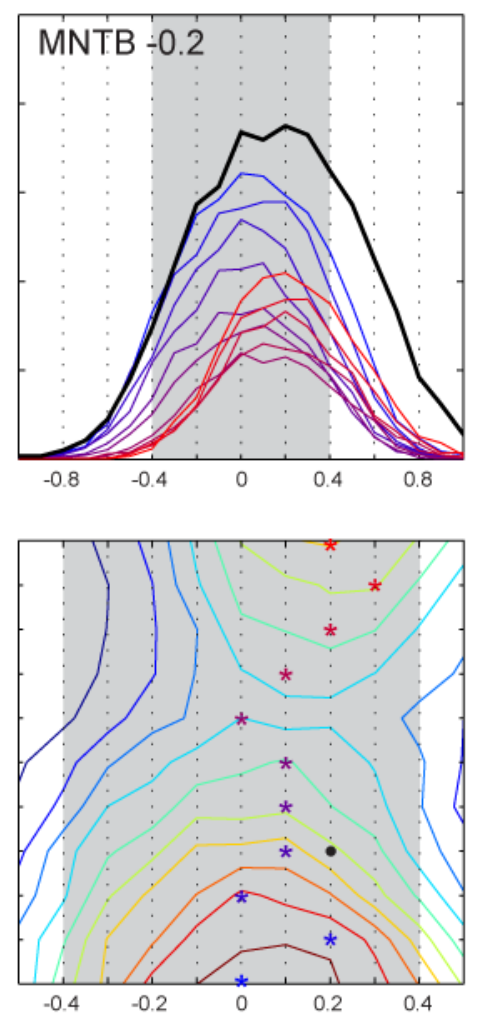

E
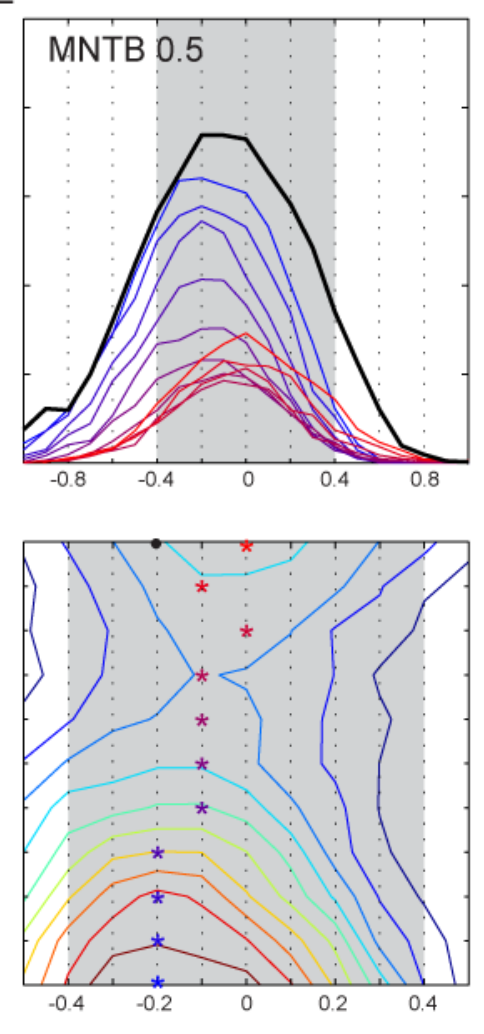

C
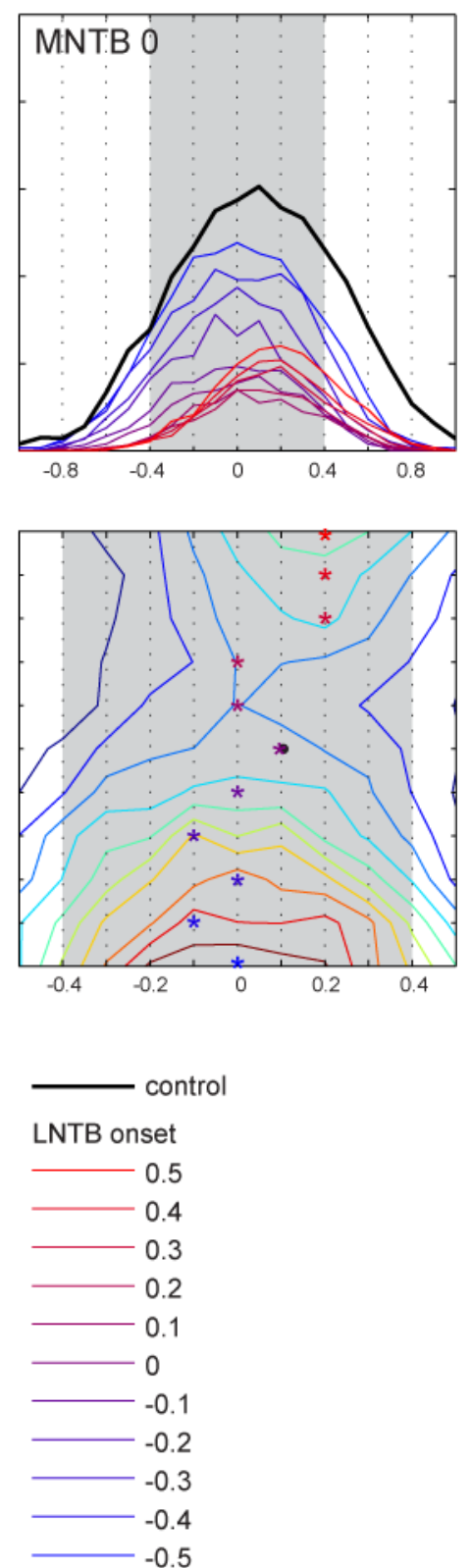
Figure 3-9.

EIEI Model for a range of MNTB delays. A, MNTB leading or simultaneous with contralateral excitation (-0.4, -0.2, $0 \mathrm{~ms})$. Leading LNTB inhibition generates ITD curves centered at $0 \mathrm{~ms}$ (blue traces in top row). Lagging LNTB input (red traces) generates peak discharge rates at positive ITDs. Traces also have steeper slopes near 0 ITD. Topologic map of discharge rate (bottom row of graphs) show peak discharge rate can be shifted to more positive values than unilateral leading inhibition (black traces in upper row of graphs). Note expanded temporal scale of bottom relative to top panels. B, MNTB lagging contralateral excitation $(0.2,0.4 \mathrm{~ms})$. Shift of peak rate to negative ITD values seen with unilateral inhibition (black traces in top row) maintained or further shifted with leading LNTB inhibition (blue traces in top row). Leading LNTB inhibition shifted peak rate toward 0 ms. 
A
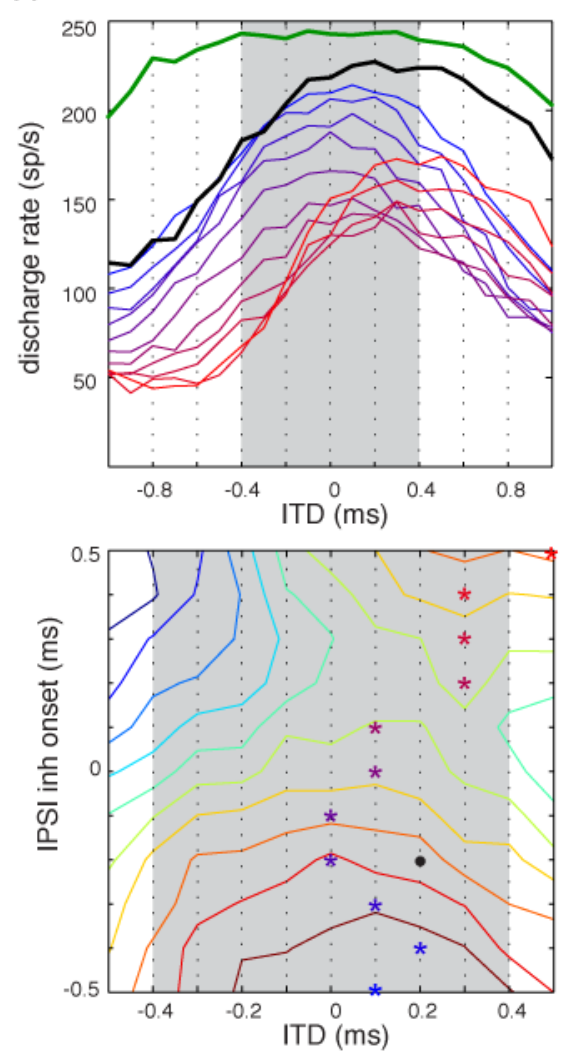

B
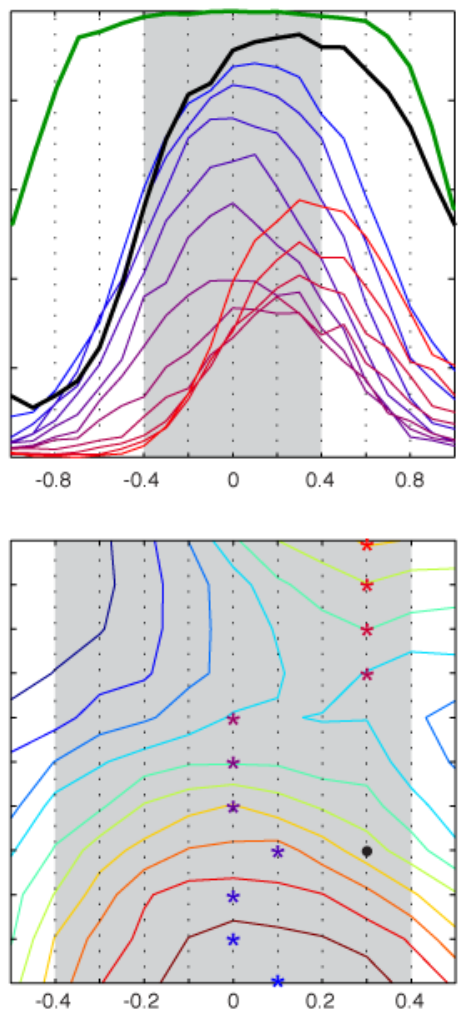

C

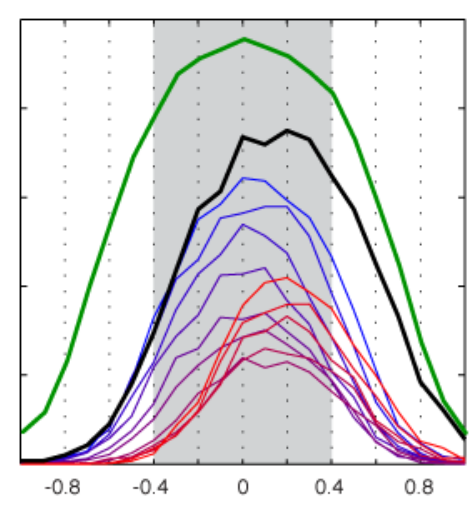

control EE

EIE onset

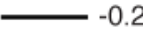

LNTB onset

0.5

0.4

0.2

- 0.1
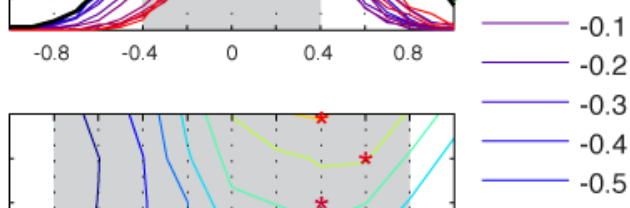

Figure 3-10.

Variation in conductance and number of inputs demonstrate advantages in El-El model compared with an EIE and EE model (black and green traces in each panel). A. Reducing the number of inputs while maintaining overall conductance of the inputs broadens the ITD curves and also makes possible the shift in ITD peak to values outside of the physiological range. B, C. Increasing conductances in excitatory inputs (panel B relative to panel C) leads to broadening of the ITD curves in every model (EE, EIE and EIEI). Shifts in peak discharge rate outside the physiological range, in $\mathrm{B}$, are attributed to inhibition carving out a larger area the of EE ITD curve. In all three scenarios, adding a second inhibitory input resulted in the steepest slope in the ITD curve near 0 ms ITD. 
A

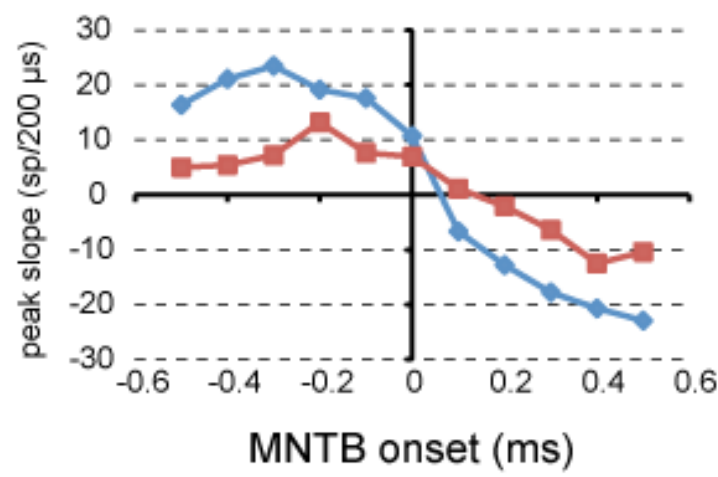

B

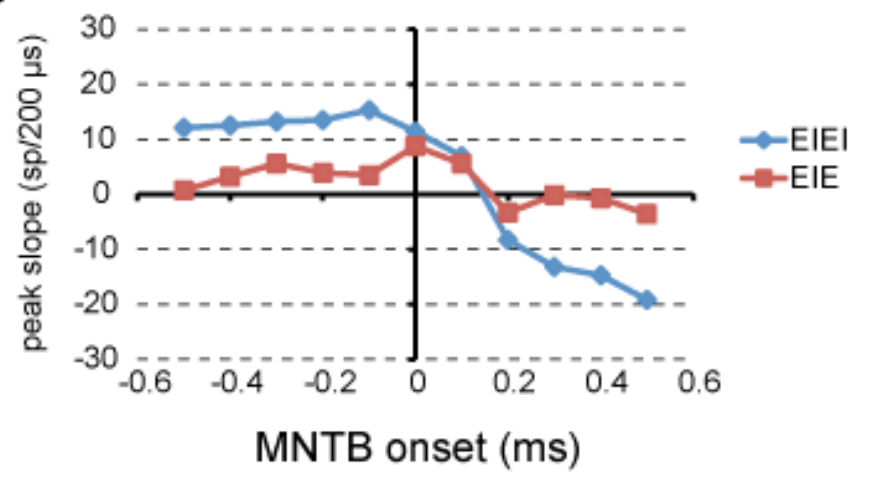

Figure 3-11.

EIEI models generate ITD curves with steeper slopes, calculated from -0.1 to $+0.1 \mathrm{~ms}$ ) than EIE models. A. 10 inputs at low conductance (EPSP 7 nS; IPSP 10 nS), B. 4 inputs at high conductance (EPSP 25 nS; IPSP 50 nS). 


\section{REFERENCES}

Adams JC (1983) Cytology of periolivary cells and the organization of their projections in the cat. J Comp Neurol 215:275-289.

Agmon-Snir H, Carr CE, Rinzel J (1998) The role of dendrites in auditory coincidence detection. Nature 393:268-272.

Beckius GE, Batra R, Oliver DL (1999) Axons from anteroventral cochlear nucleus that terminate in medial superior olive of cat: observations related to delay lines. J Neurosci 19:3146-3161.

Blackburn CC, Sachs MB (1989) Classification of unit types in the anteroventral cochlear nucleus: PST histograms and regularity analysis. Journal of Neurophysiology 62:13031329.

Brand A, Behrend O, Marquardt T, McAlpine D, Grothe B (2002) Precise inhibition is essential for microsecond interaural time difference coding. Nature 417:543-547.

Brughera AR, Stutman ER, Carney LH, Colburn HS (1996) A Model with Excitation and Inhibition for Cells in the Medial Superior Olive. Auditory Neuroscience 2:219-233.

Brunso-Bechtold JK, Henkel CK, Linville C (1990) Synaptic organization in the adult ferret medial superior olive. J Comp Neurol 294:389-398.

Cant NB (1991) Projections to the lateral and medial superior olivary nuclei from the spherical and globular bushy cells of the anteroventral cochlear nucleus. New York: Raven Press.

Cant NB, Casseday JH (1986) Projections from the anteroventral cochlear nucleus to the lateral and medial superior olivary nuclei. J Comp Neurol 247:457-476.

Cant NB, Hyson RL (1992) Projections from the lateral nucleus of the trapezoid body to the medial superior olivary nucleus in the gerbil. Hear Res 58:26-34.

Chirila FV, Rowland KC, Thompson JM, Spirou GA (2007) Development of gerbil medial superior olive: integration of temporally delayed excitation and inhibition at physiological temperature. The Journal of Physiology 584:167-190.

Clark GM (1969) The ultrastructure of nerve endings in the medial superior olive of the cat. Brain Res 14:293-305.

Couchman K, Grothe B, Felmy F (2010) Medial superior olivary neurons receive surprisingly few excitatory and inhibitory inputs with balanced strength and short-term dynamics. J Neurosci 30:17111-17121.

Dasika VK, White JA, Colburn HS (2007) Simple models show the general advantages of dendrites in coincidence detection. J Neurophysiol 97:3449-3459.

Dodla R (2005) Well-Timed, Brief Inhibition Can Promote Spiking: Postinhibitory Facilitation. Journal of Neurophysiology 95:2664-2677.

Friauf E, Ostwald J (1988) Divergent projections of physiologically characterized rat ventral cochlear nucleus neurons as shown by intra-axonal injection of horseradish peroxidase. Exp Brain Res 73:263-284.

Goldberg JM, Brown PB (1969) Response of binaural neurons of dog superior olivary complex to dichotic tonal stimuli: some physiological mechanisms of sound localization. J Neurophysiol 32:613-636. 
Guinan JJ, Norris BE, Guinan SS (1972) Single Auditory Units in the Superior Olivary Complex II: Locations of Unit Categories and Tonotopic Organization. J Neuroscience 4:147-166.

Gundersen HJ, Jensen EB (1987) The efficiency of systematic sampling in stereology and its prediction. J Microsc 147:229-263.

Gundersen HJ, Jensen EB, Kiêu K, J N (1999) The efficiency of systematic sampling in stereology--reconsidered. J Microsc 193:199-211.

Hines ML, Carnevale NT (1998) The Neuron Simulation Environment.26.

Jeffress LA (1948) A place theory of sound localization. J Comp Physiol Psychol 41:35-39.

Jercog PE, Svirskis G, Kotak VC, Sanes DH, Rinzel J (2010) Asymmetric excitatory synaptic dynamics underlie interaural time difference processing in the auditory system. Plos Biol 8:e1000406.

Joris $\mathrm{P}$, Yin TCT (2007) A matter of time: internal delays in binaural processing. Trends Neurosci 30:70-78.

Joris PX, Carney LH, Smith PH, Yin TC (1994) Enhancement of neural synchronization in the anteroventral cochlear nucleus. I. Responses to tones at the characteristic frequency. Journal of Neurophysiology 71:1022-1036.

Karino S, Smith PH, Yin TC, Joris PX (2011) Axonal branching patterns as sources of delay in the mammalian auditory brainstem: a re-examination. J Neurosci 31:3016-3031.

Kopp-Scheinpflug C, Tolnai S, Malmierca M, Rübsamen R (2008) The medial nucleus of the trapezoid body: Comparative physiology. Neuroscience.

Kuwabara N, Zook JM (1992) Projections to the medial superior olive from the medial and lateral nuclei of the trapezoid body in rodents and bats. J Comp Neurol 324:522-538.

Leibold C (2010) Influence of inhibitory synaptic kinetics on the interaural time difference sensitivity in a linear model of binaural coincidence detection. J Acoust Soc Am 127:931-942.

Lindsey BG (1975) Fine structure and distribution of axon terminals from the cochlear nucleus on neurons in the medial superior olivary nucleus of the cat. J Comp Neurol 160:81-103.

Mathews PJ, Jercog PE, Rinzel J, Scott LL, Golding NL (2010) Control of submillisecond synaptic timing in binaural coincidence detectors by K(v)1 channels. Nat Neurosci 13:601-609.

McAlpine D, Jiang D, Palmer AR (2001) A neural code for low-frequency sound localization in mammals. Nat Neurosci 4:396-401.

Moore JK, Moore RY (1971) A comparative study of the superior olivary complex in the primate brain. Folia Primatol (Basel) 16:35-51.

Morest DK (1968) The collateral system of the medial nucleus of the trapezoid body of the cat, its neuronal architecture and relation to the olivo-cochlear bundle. Brain Res 9:288-311.

Moushegian G, Rupert AL, Gidda JS (1975) Functional characteristics of superior olivary neurons to binaural stimuli. J Neurophysiol 38:1037-1048.

Paolini AG, FitzGerald JV, Burkitt AN, Clark GM (2001) Temporal processing from the auditory nerve to the medial nucleus of the trapezoid body in the rat. Hear Res 159:101-116. 
Pecka M, Brand A, Behrend O, Grothe B (2008) Interaural time difference processing in the mammalian medial superior olive: the role of glycinergic inhibition. J Neurosci 28:69146925.

Rothman JS, Manis PB (2003a) Differential expression of three distinct potassium currents in the ventral cochlear nucleus. J Neurophysiol 89:3070-3082.

Rothman JS, Manis PB (2003b) Kinetic analyses of three distinct potassium conductances in ventral cochlear nucleus neurons. J Neurophysiol 89:3083-3096.

Russell FA, Moore DR (2002) Ultrastructural transynaptic effects of unilateral cochlear ablation in the gerbil medial superior olive. Hear Res 173:43-61.

Scheibel ME, Scheibel AB (1974) Neuropil organization in the superior olive of the cat. Exp Neurol 43:339-348.

Schwartz IR (1977) Dendritic Arrangements in Cat Medial Superior Olive. Neuroscience 2:81$\&$.

Scott LL, Mathews PJ, Golding NL (2010) Perisomatic voltage-gated sodium channels actively maintain linear synaptic integration in principal neurons of the medial superior olive. J Neurosci 30:2039-2050.

Slomianka L, West MJ (2005) Estimators of the precision of stereological estimates: an example based on the CA1 pyramidal cell layer of rats. Neuroscience 136:757-767.

Smith PH, Joris PX, Yin TC (1998) Anatomy and physiology of principal cells of the medial nucleus of the trapezoid body (MNTB) of the cat. Journal of Neurophysiology 79:31273142.

Smith PH, Joris PX, Carney LH, Yin TC (1991) Projections of physiologically characterized globular bushy cell axons from the cochlear nucleus of the cat. J Comp Neurol 304:387-407.

Spangler KM, Warr WB, Henkel CK (1985) The projections of principal cells of the medial nucleus of the trapezoid body in the cat. J Comp Neurol 238:249-262.

Spangler KM, Cant NB, Henkel CK, Farley GR, Warr WB (1987) Descending projections from the superior olivary complex to the cochlear nucleus of the cat. J Comp Neurol 259:452-465.

Spirou G, Rager J, Manis P (2005) Convergence of auditory-nerve fiber projections onto globular bushy cells. Neuroscience 136:843-863.

Spirou GA, Berrebi AS (1996) Organization of ventrolateral periolivary cells of the cat superior olive as revealed by PEP-19 immunocytochemistry and Nissl stain. J Comp Neurol 368:100-120.

Spirou GA, Berrebi AS (1997) Glycine immunoreactivity in the lateral nucleus of the trapezoid body of the cat. J Comp Neurol 383:473-488.

Spirou GA, Rowland KC, Berrebi AS (1998) Ultrastructure of neurons and large synaptic terminals in the lateral nucleus of the trapezoid body of the cat. J Comp Neurol 398:257-272.

Stotler WA (1953) An experimental study of the cells and connections of the superior olivary complex of the cat. J Comp Neurol 98:401-431.

Svirskis G, Dodla R, Rinzel J (2003) Subthreshold outward currents enhance temporal integration in auditory neurons. Biological Cybernetics 89:333-340.

Tolbert LP, Morest DK, Yurgelun-Todd DA (1982) The neuronal architecture of the anteroventral cochlear nucleus of the cat in the region of the cochlear nerve root: horseradish peroxidase labelling of identified cell types. Neuroscience 7:3031-3052. 
Tsuchitani C (1977) Functional organization of lateral cell groups of cat superior olivary complex. J Neurophysiol 40:296-318.

Tsuchitani C (1994) The brain stem evoked response and medial nucleus of the trapezoid body. Otolaryngol Head Neck Surg 110:84-92.

Warr WB (1969) Fiber degeneration following lesions in the posteroventral cochlear nucleus of the cat. Exp Neurol 23:140-155.

Warr WB (1972) Fiber degeneration following lesions in the multipolar and globular cell areas in the ventral cochlear nucleus of the cat. Brain Res 40:247-270.

Werthat F, Alexandrova O, Grothe B, Koch U (2008) Experience-dependent refinement of the inhibitory axons projecting to the medial superior olive. Dev Neurobiol 68:1454-1462.

Wiegrebe L, Meddis R (2004) The representation of periodic sounds in simulated sustained chopper units of the ventral cochlear nucleus. J Acoust Soc Am 115:1207-1218.

Yin TC, Chan JC (1990) Interaural time sensitivity in medial superior olive of cat. Journal of Neurophysiology 64:465-488.

Zhou Y, Carney LH, Colburn HS (2005) A Model for Interaural Time Difference Sensitivity in the Medial Superior Olive: Interaction of Excitatory and Inhibitory Synaptic Inputs, Channel Dynamics, and Cellular Morphology. Journal of Neuroscience 25:3046-3058. 
4. Neural Encoding of Sound in the Medial Nucleus of the Trapezoid Body 
Introduction and methods

MNTB provides a functionally important inhibitory input to the MSO (Chirila et al. 2008). For this reason, characterizing MNTB cell responses to low frequency sound as well as the low frequency modulation of high frequency sound is pertinent to understanding how phase locked inhibition shapes ITD processing in the MSO. We used a dorsal approach to access neurons in the SOC of decerebrate cats. Multiple electrode penetrations were made in a grid that covered much of the medial SOC, and resulted in electrode tracks traversing the MNTB. Reconstructions of brainstems and electrode tracks performed on 9 animals allowed us to identify tracks penetrating the MNTB in 7 experiments (example in Fig. 4-1). From these reconstructions 18 units were located within the borders of the MNTB. We used the holding time of a cell as an indicator that it was a recording near the cell body of an MNTB neuron, and not an axon. Nine units were held the entire 30-40 minutes for the stimulus protocol to be completed, and these cells are reported here. In the grid of electrode penetrations, not every penetration was explicitly recovered, so we used the recovered tracks to assign recording locations to single units recorded on the other tracks. In the brainstem reconstructions the MNTB measured at least $1 \mathrm{~mm}$ wide, medial to lateral. Since electrode penetrations $0.5 \mathrm{~mm}$ lateral of the midline penetrated the medial side of the MNTB, we assigned recording sites located between $0.5-1.5 \mathrm{~mm}$ lateral of midline and recording depths less than $8000 \mu \mathrm{m}$ to the MNTB. Based on these metrics, an additional 10 units, which were held for the entire stimulus protocol, were localized to the MNTB. The following is a report of the response characteristics from these 19 isolated units.

\section{Peri-stimulus time histograms}

A guiding consideration in all of our physiological studies was the comparison of sound-evoked neural activity in unanesthetized, decerebrate cats to existing recordings in anesthetized cats and rodents. If inhibitory neurons are more active in decerebrate animals, then we should expect differences even in responses to the simplest sounds, which are pure tones. In this section, we report PSTH categories and their properties in decerebrate cats. PSTHs were constructed using 500 repetitions of pure tone stimuli at CF, $60 \mathrm{~ms}$ in duration and at $30 \mathrm{~dB}$ and $50 \mathrm{~dB}$ above threshold. Spike times were placed into $0.1 \mathrm{~ms}$ bins. Figures 
4-2 through 4-6 show PSTHs of the 9 units directly localized to the MNTB and demonstrate the range of response categories we recorded in the MNTB cell population. Each panel of each figure shows a PSTH encompassing the entire stimulus period, a higher resolution view of the 10-40 ms segment of the stimulus period, a graph of the coefficient of variation (CV) of spike intervals during this $30 \mathrm{~ms}$ time frame, and a first-spike latency (FSL) histogram. Sound was gated on beginning $10 \mathrm{~ms}$ after the beginning of the stimulus period, so a brief period of spontaneous activity preceded the onset of the sound-evoked response. Only spikes that occurred within a time window of 12-22 ms, to capture the clear onset of the response in the PSTH, were included in the FSL histogram. First spike latency is calculated from the average spike time between the dashed vertical bars, applied just preceding and just following the onset response peak. The $\mathrm{CV}$ calculation, which is the mean of the first order interspike intervals divided by the standard deviation of intervals, requires a second spike to complete an interspike interval. Each value was plotted at the time of the first spike that defined the interval. Therefore, the calculation was only extended $30 \mathrm{~ms}$ into a $60 \mathrm{~ms}$ duration stimulus, so that each spike was followed by at least one other spike during the stimulus and an interval could be measured. The $\mathrm{CV}$ is used to quantify the regularity of spike intervals. This metric has proven useful in distinguishing primary-like from chopper PSTH categories, and in distinguishing types of chopper responses in the cochlear nucleus (Bourk, 1976; Young et al., 1988; Blackburn and Sachs, 1989).

PSTH responses of GBCs and MNTB cells have been categorized as phase-locked for low frequency tones and PL or $\mathrm{PL}_{N}$ for high frequency tones (GBC: Brownell, 1975; Rhode et al., 1983; Friauf and Ostwald, 1988; Young et al., 1988; Spirou et al., 1990; Smith et al., 1993); (MNTB: Guinan et al., 1972a, b; Tsuchitani, 1997; Kadner et al., 2006; KoppScheinpflug et al., 2008). Cells are arranged in increasing order of CF in figures 4-2 through 4-6. Three units, at $860 \mathrm{~Hz}, 913 \mathrm{~Hz}$ and $1.05 \mathrm{kHz}$, are shown in figures 4-2 and 4-3A. These cells exhibited strong phase-locking to the stimulus as evidenced by multiple peaks in the histogram at intervals that are the stimulus period. In all cells, the amplitude of the peaks decreased during the stimulus, indicating a decreasing discharge rate. Responses of all three cells to relatively loud sound at $50 \mathrm{~dB}$ re: threshold exhibited a range of discharge rates (101 $-281 \mathrm{sp} / \mathrm{s}$ ) after their onset response, although the rate in 2/3 cells continued to decrease throughout the sound. Spontaneous rates ranged from 0 to $18.4 \mathrm{sp} / \mathrm{s}$. The $\mathrm{CV}$ trended to 
values greater than 0.5 , indicating that stimulus intervals were skipped between spikes in somewhat random fashion, even though intervals occured at multiples of the stimulus period. The first spike latency (FSL) histograms showed that the first spike occurred at sound onset for nearly all stimulus presentations, and that the standard deviation of FSL can be quite low ( $<0.04 \mathrm{~ms}$ ). The two lowest CF cells had first spike latencies that approached $7 \mathrm{~ms}$.

Cells tuned to higher CF's had response types that were primary-like with notch $\left(\mathrm{PL}_{N}\right.$, Fig. 4-3, B; 4-6), chopper (Fig. 4-4, B; 4-5, A) and a hybrid type of response that we term primary-like with multiple notches ( $\mathrm{PL}_{\mathrm{MN}}$, Fig. 4-4, A; 4-5, B). Similarities in PSTHs evident in figures 4-4 and 4-5 imply the difficulties of using a qualitative method of PSTH categorization. Quantification of discharge regularity by the $C V$ distinguished $P L_{N}$ and $P L_{M N}$ from chopper responses, and sustained (Chop-S) from transient chopper (Chop-T) responses.

The $\mathrm{PL}_{N}$ response illustrates this approach (Fig. 4-3, B). A clear notch in the response following the onset is evident in the full histogram and the expanded view of the early part of the response. The driven rate at $50 \mathrm{~dB}$ re: threshold was sustained at $154 \mathrm{sp} / \mathrm{s}$ and the spontaneous rate was $19.6 \mathrm{sp} / \mathrm{s}$. The FSL histogram shows a response at sound onset to nearly all stimulus presentations, with a small standard deviation, and at a shorter latency than the lower CF cells. The CV analysis remained greater than 0.5 throughout the sound, indicating an irregular pattern of interspike intervals that is characteristic of PL responses in auditory nerve and $\mathrm{VCN}$ and $P L_{N}$ responses in $\mathrm{VCN}$. The cell shown in figure 4-4A shares many of these characteristics, in that the sustained rate appeared constant after the onset and the FSL had a smaller value and small standard deviation. However, an expanded view of the PSTH reveals four peaks and notches before transitioning to a sustained appearance. Despite the early peaks in the PSTH, the CV had values near 1 , and maintained values $>0.5$ during the sustained portion of the response. The cells shown in figures 4-6 and 4-5B are classified as $P L_{N}$ and $P L_{M N}$, respectively, because of the number of notches and $C V$ values maintained at $>0.5$ during the sound. The cell in figure $4-6$ is notable for its very low driven rate, even at $50 \mathrm{~dB}$ re: threshold.

The utility of the CV analysis is further illustrated by the Chop-Sustained(S) response in figure 4-4B. Three clear peaks occured during the early portion of the response before reaching a sustained rate of $279 \mathrm{sp} / \mathrm{s}$. The first spike latency had a slightly larger standard deviation than the PL cells, but was still very precise. The distinction among response types 
was most easily made by the $\mathrm{CV}$, which remained at values $<0.5$ throughout the response, indicating greater regularity in intervals than is seen with $P L_{N}$ and $P L_{M N}$ cells. The cell depicted in figure 4-5A also had peaks early in the response, with six peaks in the response at the beginning of the sound. The FSL was of short latency and similar in precision to the Chop-S cell, and had the highest driven rate in this group of 9 cells. The CV began at low values before settling at a value between 0.5 and 0.6 , indicating less regularity during the latter portion of the response. Based upon the transition from low to high CV feature, we classified the cell as Chop-Transient(T).

Ten single unit recordings that were mapped, using nearby tracks (as described previously), to the MNTB demonstrated phase-locked, $\mathrm{PL}_{\mathrm{N}}$, or Chop-S responses. Phaselocking was observed in 3 units having CFs ranging from $0.4-2.37 \mathrm{kHz}$. In one cell $(\mathrm{CF}=1.4$ $\mathrm{kHz}$ ) weak phase locking was observed within a Chop-S discharge pattern. The remaining 6 units had $\mathrm{PL}_{\mathrm{N}}$ responses often having multiple peaks in the initial response but lacked the distinct notching seen in $\mathrm{PL}_{\mathrm{MN}}$ responses. In total we recorded 6 phase-locked, $8 \mathrm{PL}_{\mathrm{N}}, 2$ $\mathrm{PL}_{\mathrm{MN}}, 2$ Chop-S and 1 Chop-T cell. A summary of response characteristics is shown in table 4-1.

First spike latency

Responses to Tones. We measured the response latencies of MNTB neurons to tones and clicks in order to provide baseline information about the timing of activation of MNTB, as a template for further investigation of MSO and LNTB cell latency using our acoustic system. We plotted first spike latencies (FSL) of the 19 MNTB cells that were categorized by PSTH (Fig. 4-7A) by the log (CF) of the cell. On this scale, latencies of all response types were close to a regression line that decreased in FSL with increasing log (CF) $(p<.01)$. Phaselocked cells had the lowest CF and longest FSL but also the greatest range of values (> 2ms). Numbers of Chop-S, Chop-T and PLMN were small, so statistical analysis is difficult. It is noteworthy that Chop-S and ChopT values were above the regression line by up to $0.5 \mathrm{~ms}$ and $P L_{M N}$ were above the regression line but within the $P L_{N}$ population.

Responses to Clicks. Click stimuli are studied as a representation of neural responses to acoustic transients which, in contrast to pure tones, are a consistent element of the natural world. This population includes 15 of the 19 units reported above, as click stimuli were a 
recent addition to our stimulus protocol. An additional 8 units (triangles), which were localized to MNTB but were only briefly held ( 5 minutes), were plotted with the characterized cells to gain a better estimate of the range of latencies in the MNTB. FSL to clicks were shorter than FSL to pure tones for each cell studied with both stimuli. The dependence of latency with $\log (\mathrm{CF})$ was less evident than with tones, yet retained a slight negative dependence between these variables. A regression line was fit to only data points of PSTH-categorized cells. Phase-locked cells had the longest latencies as well as the greatest spread in FSL (average $=5.18 \pm 1.02 \mathrm{~ms}, \mathrm{n}=6$ ), yet could have latencies as short as $4 \mathrm{~ms}$. FSL for all other response types were tightly distributed $(4.51 \pm 0.35 \mathrm{~ms}, \mathrm{n}=17)$ and were indistinguishable among response types.

\section{Spontaneous discharge rate}

Because GBCs are the primary source of input to MNTB cells, one might expect that spontaneous discharge rate and maximum sustained discharge rate for these two cell types would be similar (Fig. 4-8). Thus, we calculated both the maximum sustained discharge rate and the spontaneous discharge rate from the same stimuli used to generate PSTHs.

Spontaneous rate was calculated from the last $50 \mathrm{~ms}$ of the sweep and $180 \mathrm{~ms}$ after the end of the stimulus tone (see spontaneous rate bars in Figs., 4-2 through 4-6 for an examples). Phase locked units had spontaneous discharge rates which varied from 0 - $40 \mathrm{sp} / \mathrm{s}$ (Fig. 48A) Spontaneous rates for Chop-S Chop-t and $P L_{M N}$ were all below $20 \mathrm{sp} / \mathrm{s}$ in contrast to $\mathrm{PL}_{\mathrm{N}}$ units which had spontaneous discharge rates ranging from $20 \mathrm{sp} / \mathrm{s}$ to over $100 \mathrm{sp} / \mathrm{s}$. Spontaneous discharge rate for cells having a $\mathrm{CF}<2 \mathrm{kHz}$ (5 phase-locking units and 1 chopper unit) was less than $20 \mathrm{sp} / \mathrm{s}$. Low spontaneous discharge rates were observed at all CFs; however high spontaneous discharge rates were observed in high CF MNTB cells. These ranges of spontaneous discharge rate are consistent with previously published results of the MNTB and their primary inputs, GBC's of the AVCN (Guinan et al., 1972b; Spirou et al., 1990; Tsuchitani, 1997; Smith et al., 1998)

\section{Maximum sustained discharge rates}

For comparison to GBCs we measured maximum sustained discharge rates of cells plotted by response type for 19 units localized to the MNTB. Maximum sustained discharge 
rates were calculated after the initial response burst ( $20 \mathrm{~ms}$ after stimulus onset) had subsided over approximately the last $40 \mathrm{~ms}$ of the stimulus length. Maximum sustained discharge rates ranged from 96 - 381 sp/s but averaged 230 sp/s (Fig. 4-8B). Phase-locking cells had sustained discharge rates which ran the spectrum. Perfect entrainment observed in GBCs by Joris et al. was not evident in the lowest frequency MNTB cell $(\mathrm{CF}=400 \mathrm{~Hz})$ as the maximum sustained discharge rate was $<150 \mathrm{sp} / \mathrm{s}$ (1994b). Sustained discharge rates for the other types of PSTH responses could not be distinguished from the population. No correlations could be drawn between maximum sustained discharge rate and CF or method by which the cells were localized to the MNTB (data not shown).

\section{Phase-locking in MNTB cells}

Low CF MSO cells receive phase-locked excitatory inputs from SBCs and phaselocked inhibitory inputs from MNTB cells (Beckius et al., 1999; Karino et al., 2011). Studies of GBCs, the main excitatory input to MNTB cells, have revealed higher vector strengths than are present in auditory nerve fibers (Joris et al., 1994b; Joris et al., 1994a). Values greater than 0.9 were considered enhanced phase-locking. Modeling studies have demonstrated that higher vector strengths can be due to convergence of multiple ANFs onto a single GBC (Spirou et al., 2008). High CF ANFs and bushy cells cannot phase-lock at CF, but do phaselock to low frequency stimuli in the tail of their response maps. In fact, high CF GBCs have higher VS than low CF units (Joris et al., 1994b; Joris et al., 1994a). To examine the effects that an intact inhibitory circuit has on neural encoding in the MNTB, we measured phaselocking of low and high CF cells to a $500 \mathrm{~Hz}$ stimulus, and low CF units to a range of low frequency stimuli across intensity. For all cells at nearly all intensities, VS approached but did not exceed 0.9, and therefore did not demonstrate enhanced phase-locking (Fig. 4-9A). For most cells, VS did not change with increasing intensity (Fig. 4-9B) but values did increase in some instances (Fig. 4-9C). For one cell, values decreased due to splitting of the response phase between two values (Fig. 4-9D).

Three low CF cells were studied across a range of frequencies and intensities (Fig. 410). For most stimulus frequencies, phase-locking was not enhanced. Two of the three cells had higher VS for one stimulus frequency. Phase PSTHs had Gaussian-like shapes when vector strengths were high (Fig. 4-10 B, C and D). Low vector strength resulted from 
sinusoidally shaped phase PSTHs (Fig. 4-10 col. C, rows 1 and 3), peak splitting ( $2^{\text {nd }}$ row of columns $B$ and $C$ in Fig. 4-10) and onset burst responses buried in the phase PSTH (Fig. 410D row 3 ).

\section{Envelope coding of sinusoidally amplitude modulated (SAM) tones}

Sounds in the natural environment are rarely pure tone and often are complex in the frequency domain as well as the temporal domain. While natural sounds would be the ideal stimuli for examining the response properties of cells in the SOC it would be too difficult to dissect the many frequency and temporal features that contribute to neuronal responses. Because high frequency sounds are transduced into a signal containing no phase information, it has been postulated that high CF MSO cells utilize the temporal envelope of high frequency stimulus to code ITDs. To examine the temporal coding of high frequency sounds we reasoned that the use of a stimulus containing a repeating temporal feature, i.e. sinusoidally amplitude modulated (SAM) tone, would allow us to investigate envelope coding in high frequency MNTB cells. We presented SAM tones to units having CFs $>5 \mathrm{kHz}$ at modulation frequencies ranging from $6.1-6250 \mathrm{~Hz}$. To avoid interference with $60 \mathrm{~Hz}$ electrical noise, modulation frequencies were multiples that were indivisible by 60 (i.e. $\mathrm{mf}=$ $6.1,12.2,24.4,48.8,97.7,195.3,390.6,781.3,1562.5,3125,6250)$ but for ease of reference are rounded up to the nearest $5,10,50$ or 100 . This range of modulation frequencies (MF) is broader than is typically presented in studies of SAM tones. The response to SAM tones was quantified as the VS, and plots of VS across modulation frequency, termed temporal modulation transfer functions (tMTF), were generated. We focused sequentially on the low modulation frequency, peak, and high modulation frequency rolloff responses. Six cells were studied using the complete protocol for SAM tones.

At low MF, all cells exhibited decreased vector strength with increasing intensity (example shown inside oval in Fig. 4-11C). At low intensity and at the lowest MF of $6 \mathrm{~Hz}, 3 / 6$ cells had VS exceeding 0.7 . The response at such low MF is essentially a detection of when the intensity exceeds threshold. Increasing intensity resulted in lower VS. This result is understandable, because the stimulus will exceed intensity threshold over a larger portion of the stimulus period. At high intensity, all cells showed increased VS with increasing MF, and VS reached peak values between $100-400 \mathrm{~Hz}$. Because these tMTFs have a peak, they are 
referred to as band pass in shape. At lower intensity, cells that had large VS values at $6 \mathrm{~Hz}$ MF showed less of an increase in VS with increasing MF. In the extreme, VS changed by less than 0.1 , and the shape of the tMTFs more closely resembled a low pass filter (Fig. 411B, $80 \mathrm{~dB}$ att.).

In $3 / 6$ cells the tMTFs for all intensities converged to the same VS value at the peak of the function (Fig. 4-11 A, B, E). In one cell, the peak may have been obscured by a drop in VS at one value (200 Hz in Fig. 4-11E). This drop is due to peak splitting in the period histogram (Fig. 4-12C, arrows). With increasing intensity, one of the peaks retained its phase value and the other peak shifted its phase value into greater alignment. A similar response occurred, also at $200 \mathrm{~Hz}$ MF, for the cell shown in figure 4-11D.

The rolloff in VS was steeper at high than at low MF, and values were similar across intensity for $5 / 6$ cells. VS declined to near zero, and declined to a value of 0.1 at MFs between 1-2 kHz. For the highest CF cell in this sample (35 kHz, Fig. 4-11F), VS was greater than 0.1 at an MF of $3 \mathrm{kHz}$. This cell showed reduced VS with increased intensity across all MFs, such that VS values for different intensities did not align at the peak or the rolloff frequencies. 


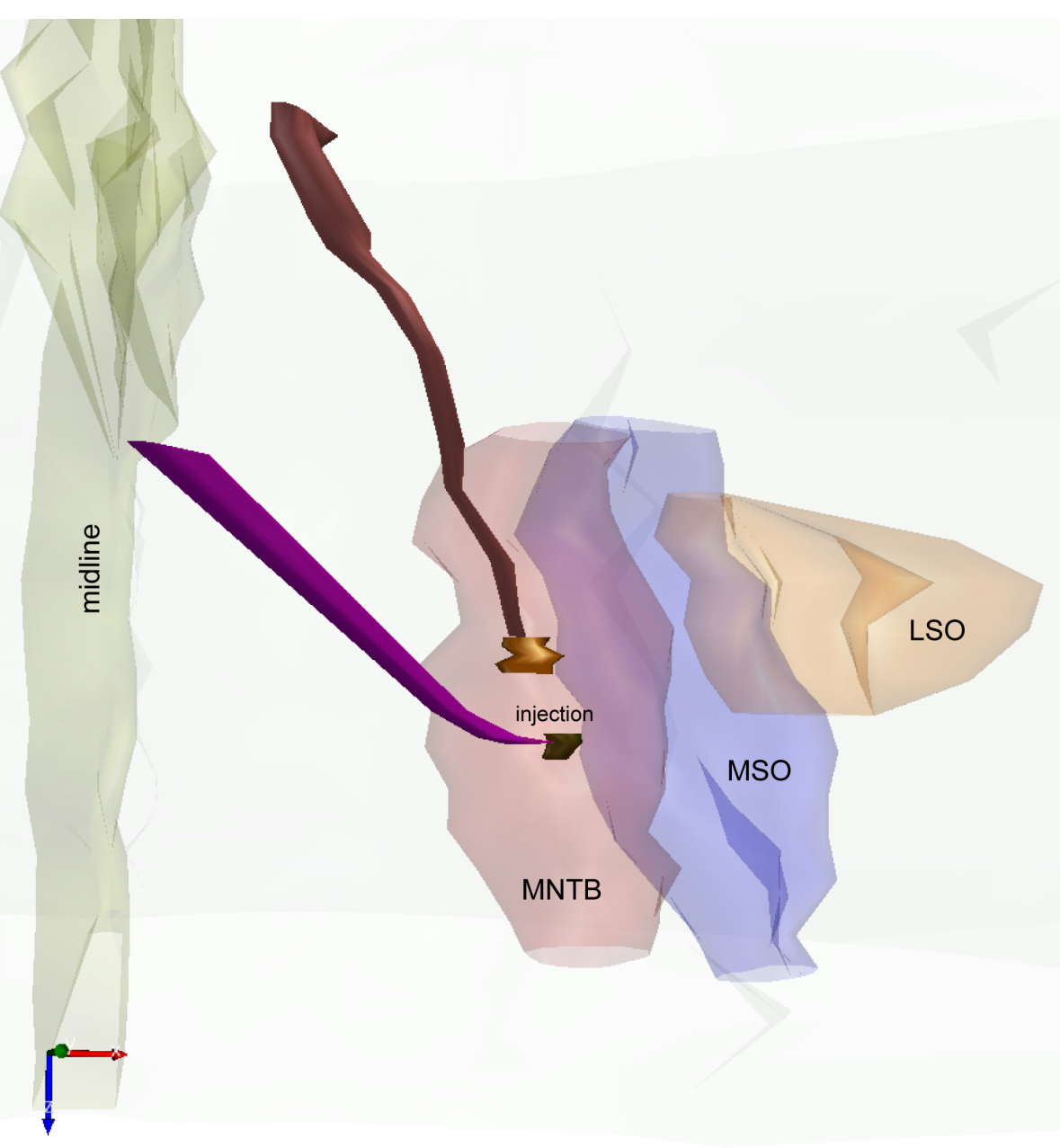

Figure 4-1.

3-dimensional reconstruction of the brainstem and SOC. Electrode tracks (brown and purple) are shown penetrating the MNTB (red) in this horizontal view. Injections marked in these reconstructions are used as reference points so that penetrations performed in the experiment can be associated with their reconstructed counterpart. Midline is shown in olive, MSO in blue and LSO in orange. For orientation, the blue and red arrows, located on the bottom left hand, point in the caudal and lateral directions respectively. The MNTB and MSO are approximately $3 \mathrm{~mm}$ in length in the rostro-caudal dimension. 
A

Characteristic frequency $=860 \mathrm{~Hz} @ 50 \mathrm{~dB}$ re: $\theta$

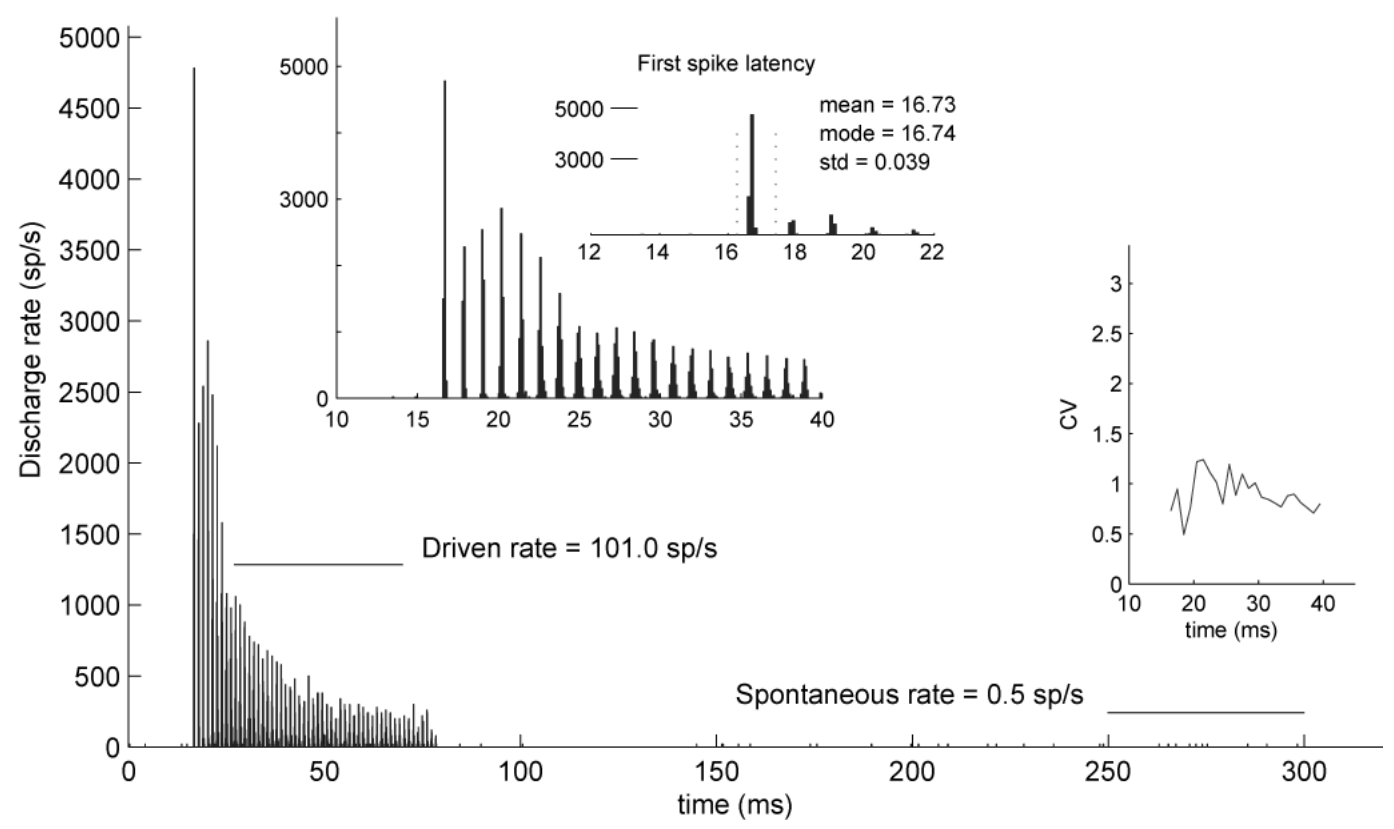

B

Characteristic frequency $=913 \mathrm{~Hz} @ 50 \mathrm{~dB}$ re: $\theta$

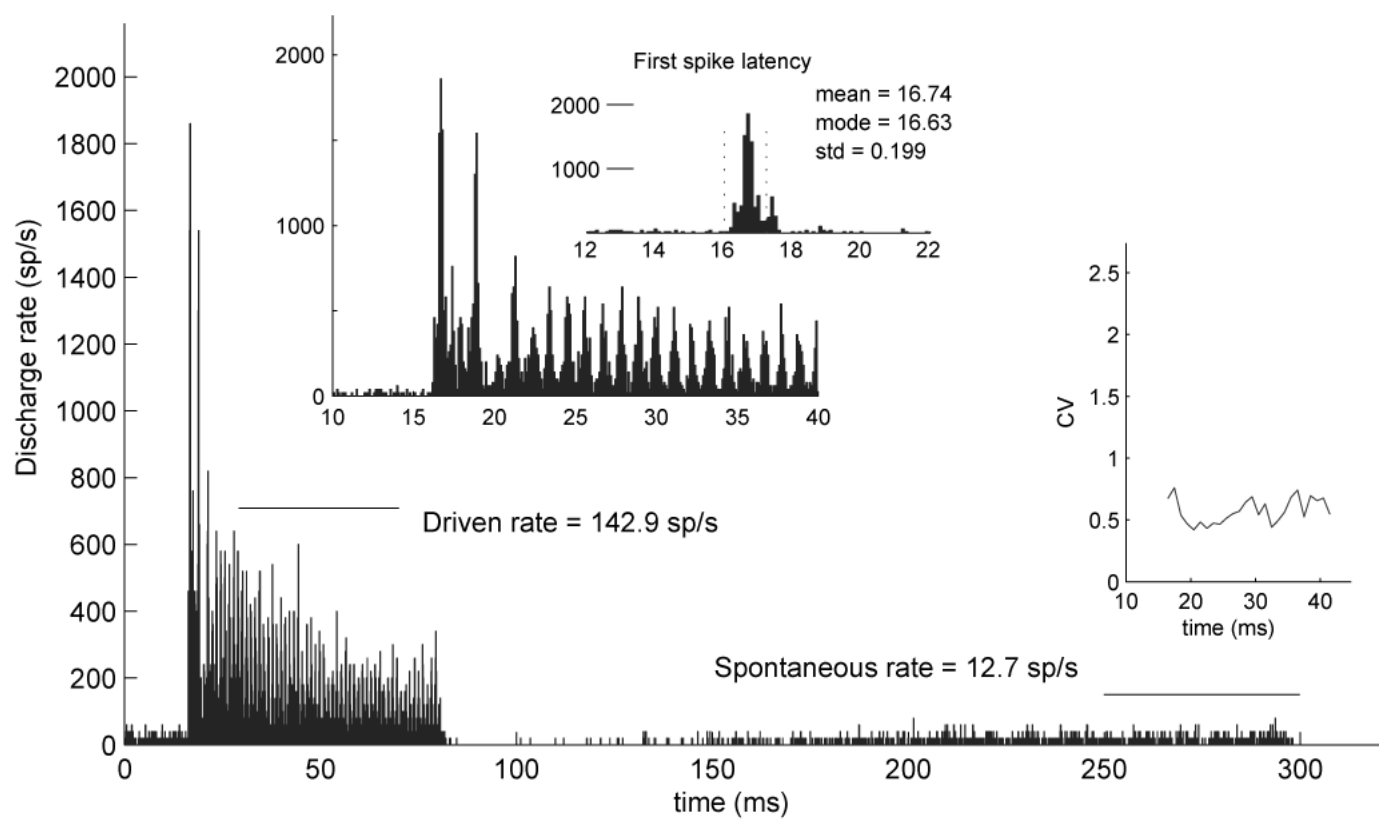

Figure 4-2.

Phase locked responses for cells having low CFs. 
A

Characteristic frequency $=1.05 \mathrm{kHz} @ 50 \mathrm{~dB}$ re: $\theta$

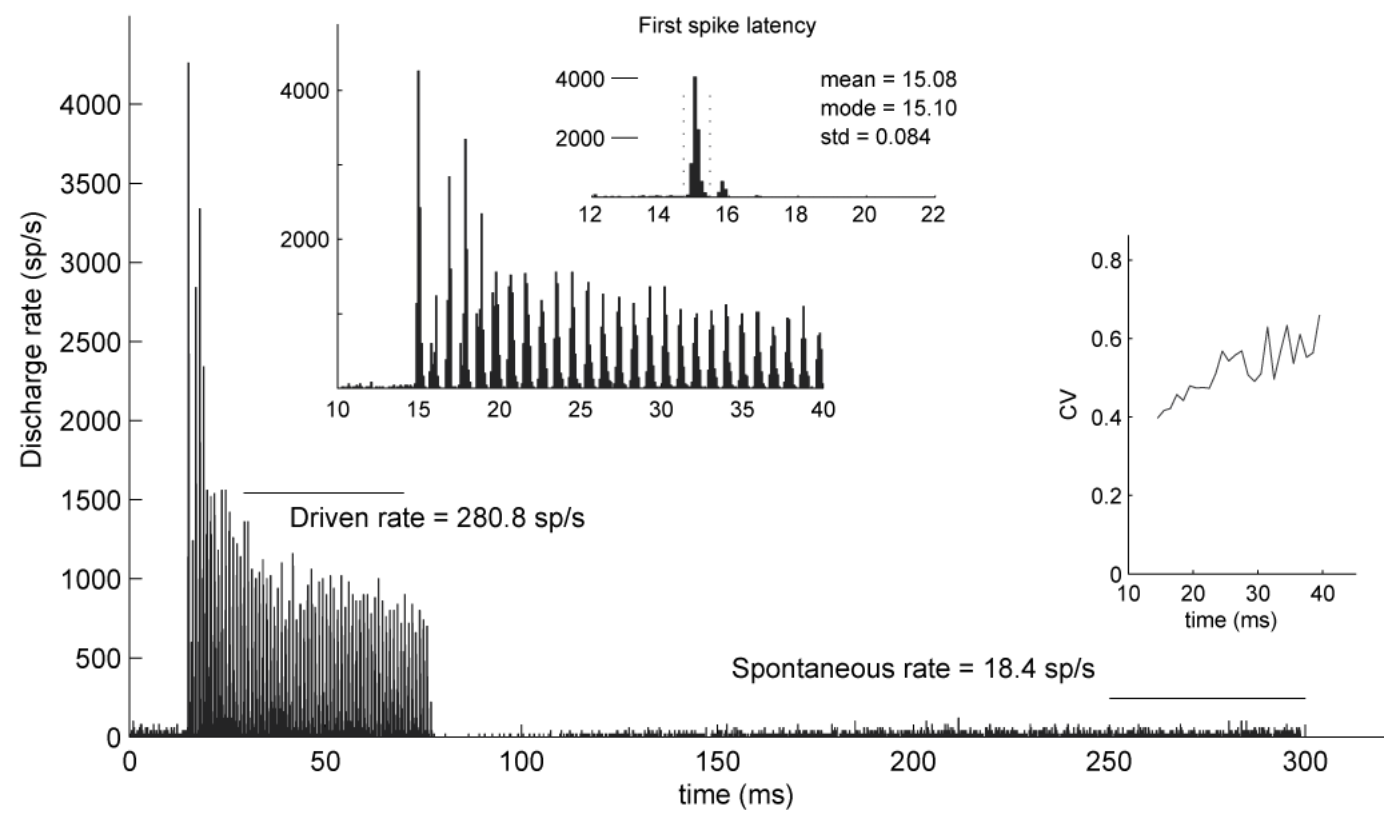

B Characteristic frequency $=6.4 \mathrm{kHz} @ 50 \mathrm{~dB}$ re: $\theta$

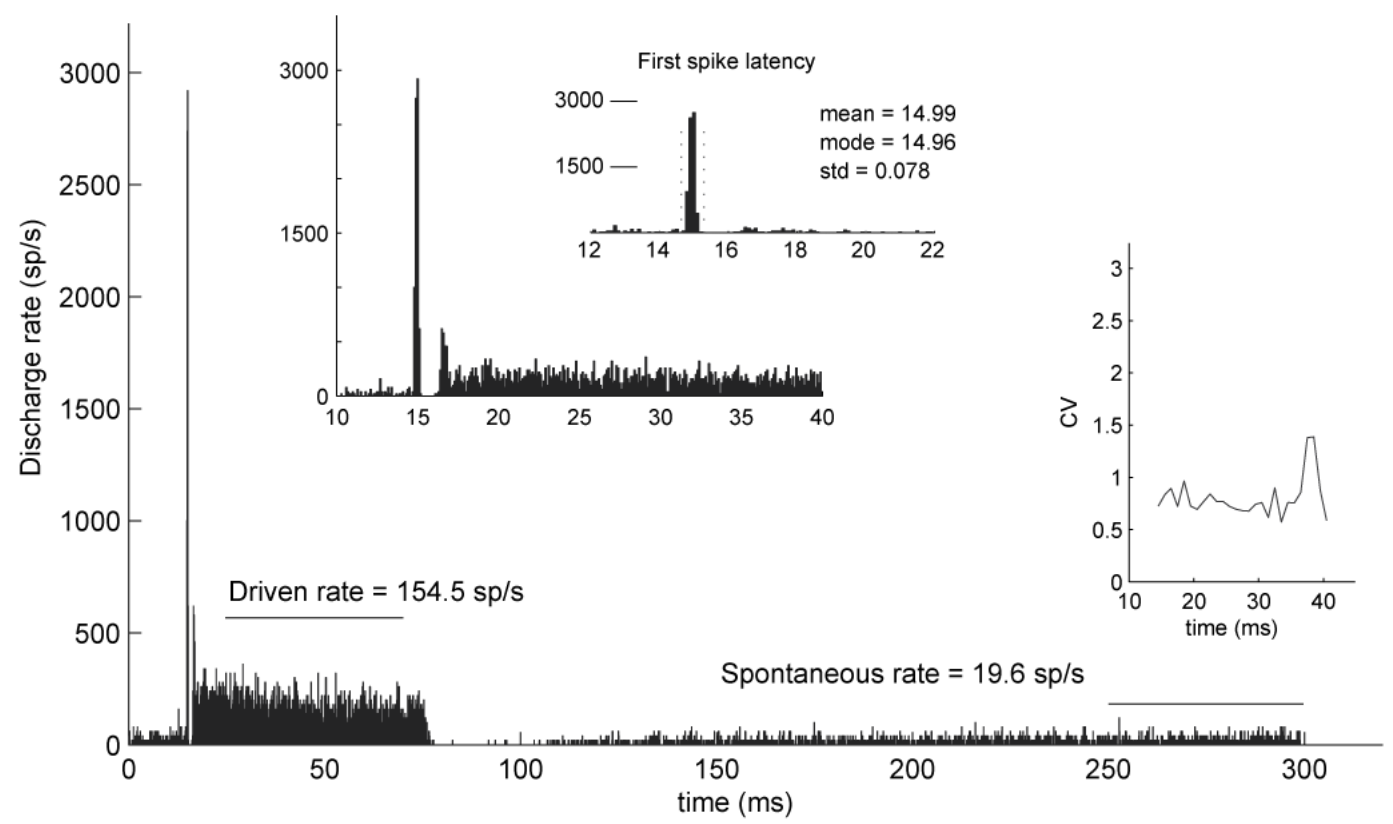

Figure 4-3.

Phase locked and primary-like with notch PSTHs 
A

Characteristic frequency $=7.7 \mathrm{kHz} @ 50 \mathrm{~dB}$ re: $\theta$

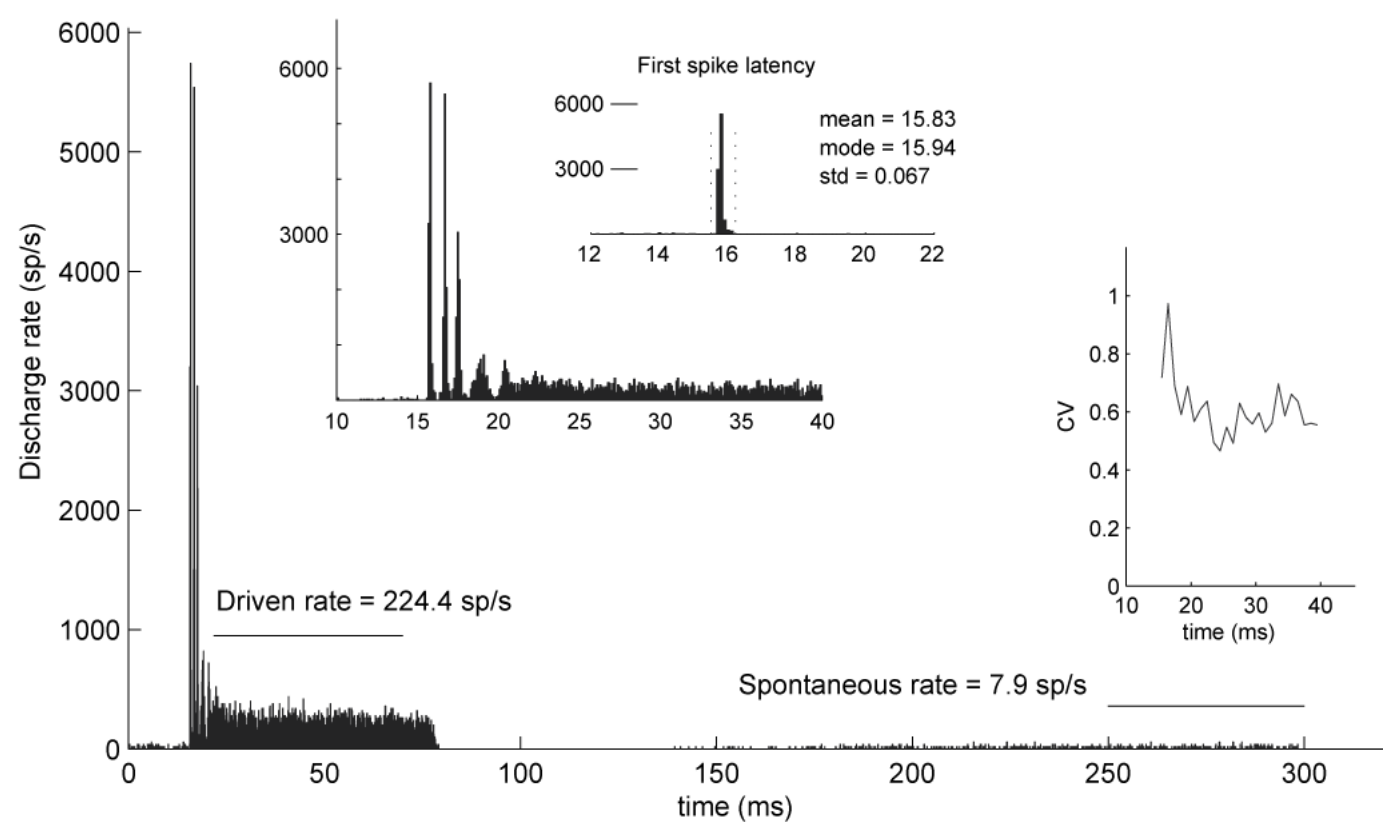

B Characteristic frequency $=8 \mathrm{kHz} @ 50 \mathrm{~dB}$ re: $\theta$

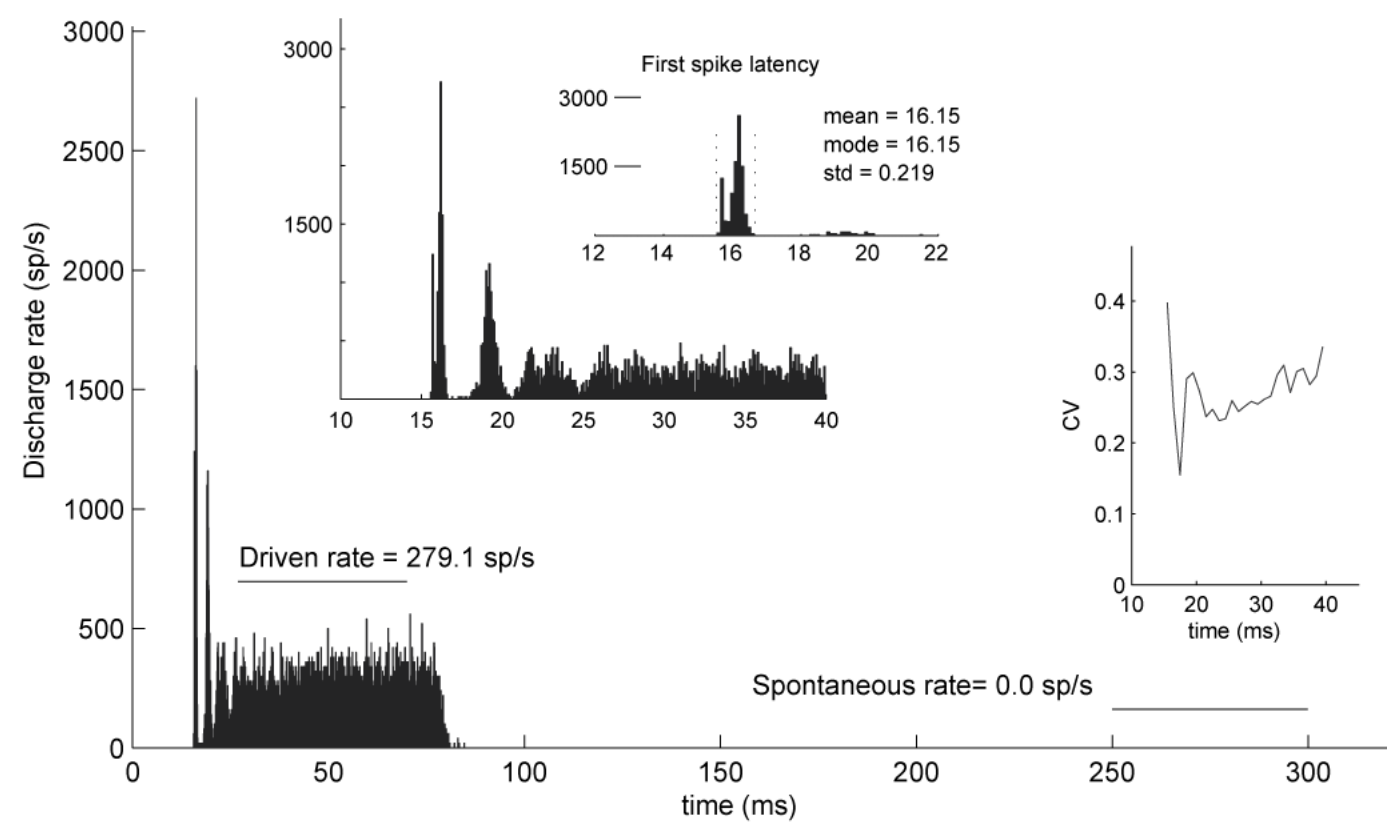

Figure 4-4.

Primary-like with multiple notches $\left(\mathrm{PL}_{\mathrm{MN}}\right)$ and Sustained-Chopper (Chop-S) PSTHs. Response in panel $A$ is unusual in that by standard definition of a PSTH it would be categorized as a Chopper response, possibly a transient chopper; however, CV and 
interspike interval histogram indicate that the response shares more in common with $\mathrm{PL}_{N}$ units. 
A

Characteristic frequency $=17.6 \mathrm{kHz} @ 50 \mathrm{~dB}$ re: $\theta$

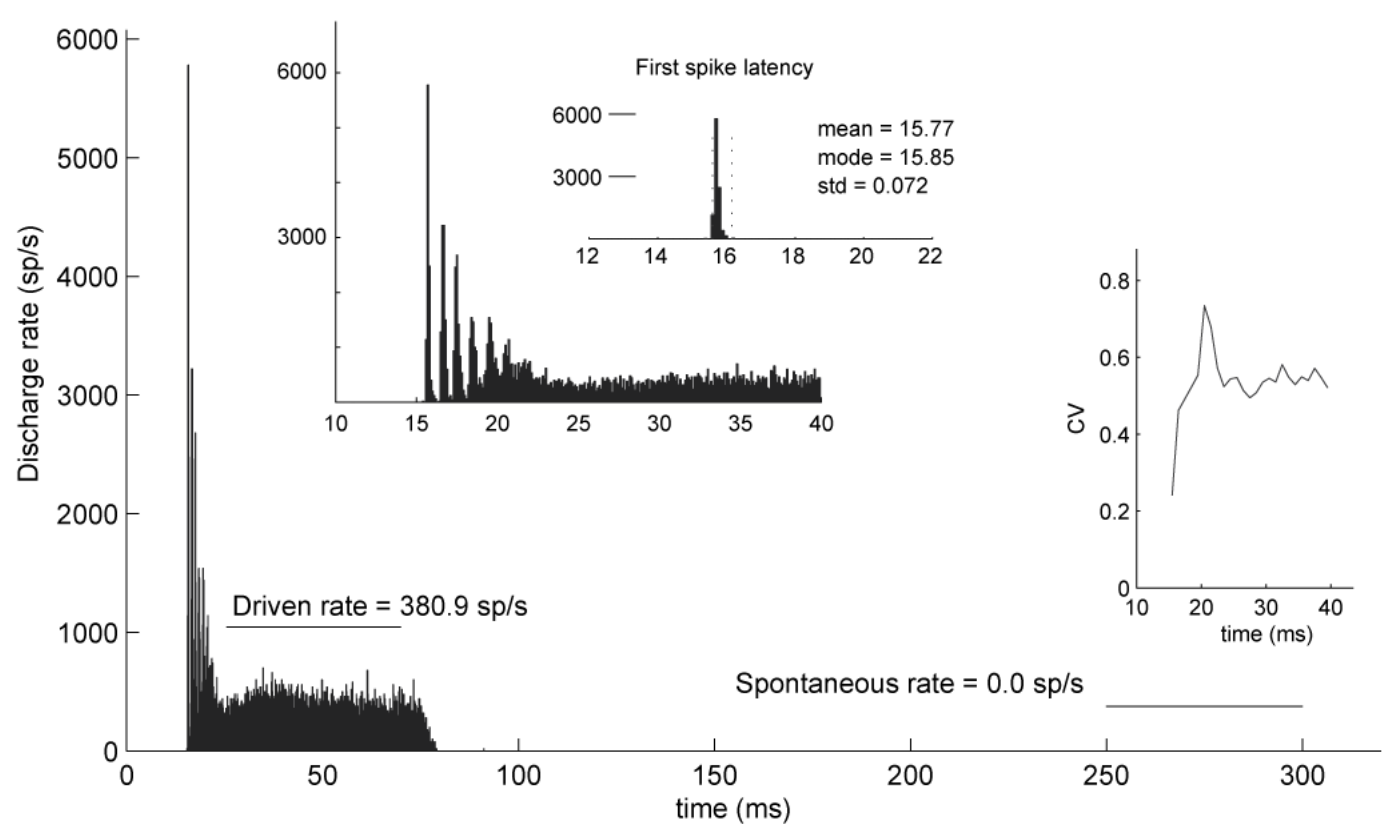

B Characteristic frequency $=18.9 \mathrm{kHz} @ 50 \mathrm{~dB}$ re: $\theta$

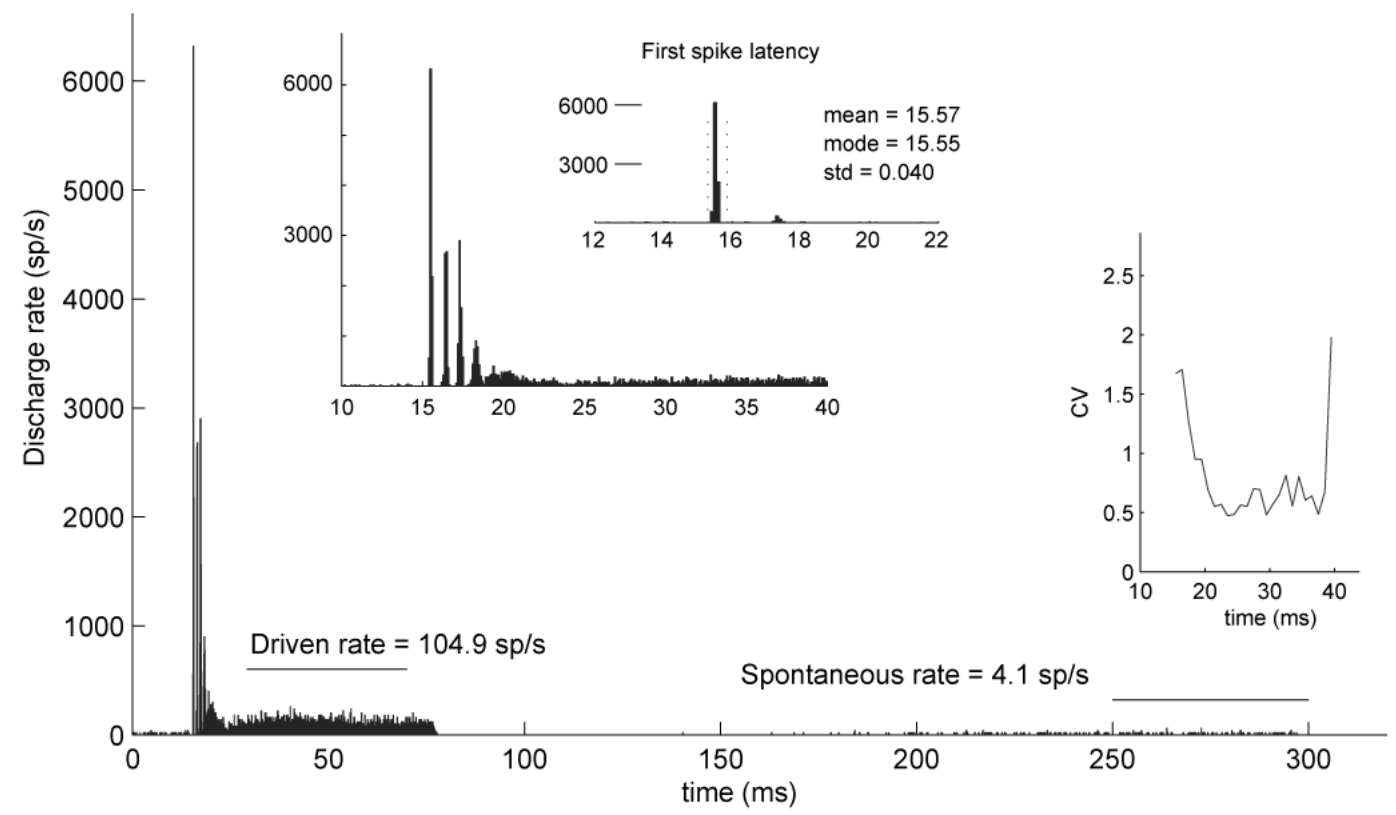

Figure 4-5.

Transient Chopper (Chop-T) and Primary-like with multiple notches ( $\left.P L_{M N}\right)$ PSTHs. Subtle difference between these units demonstrate the limitations of using qualitative PSTH categorization as the only method of identification. 
Characteristic frequency $=35 \mathrm{kHz} @ 50 \mathrm{~dB}$ re: $\theta$

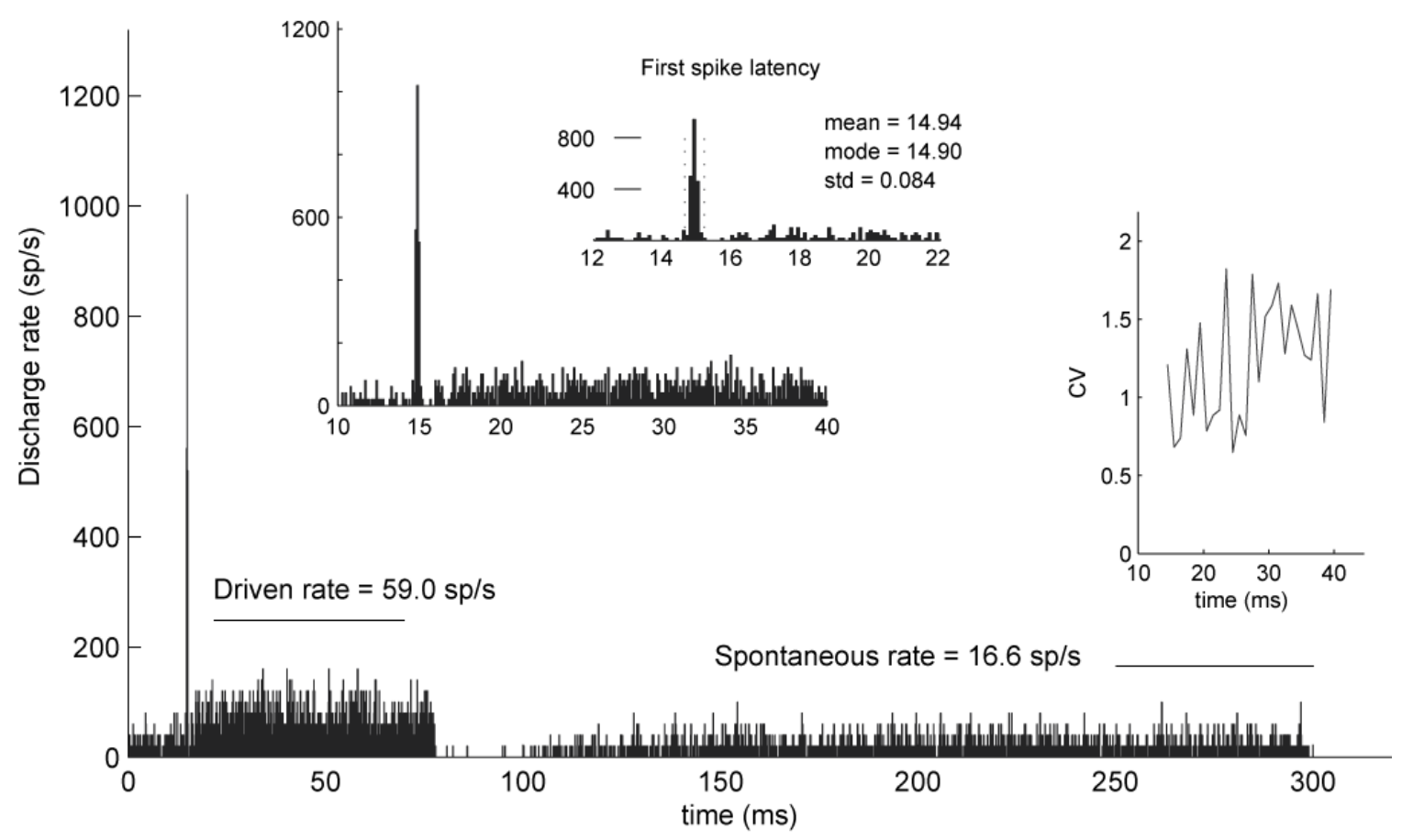

Figure 4-6.

Primary-like with notch PSTH. 


\begin{tabular}{|c|c|c|c|c|c|c|c|c|c|}
\hline \multirow{2}{*}{$\begin{array}{l}\mathrm{CF} \\
(\mathrm{kHz})\end{array}$} & \multicolumn{3}{|c|}{ PSTH type } & \multirow[b]{2}{*}{ Chop-S } & \multirow[b]{2}{*}{ Chop-T } & \multirow{2}{*}{$\begin{array}{c}\text { spontaneous } \\
\text { discharge rate } \\
\text { (sp/s) }\end{array}$} & \multirow{2}{*}{$\begin{array}{c}\text { maximum } \\
\text { sustained rate } \\
(\mathrm{sp} / \mathrm{s})\end{array}$} & \multirow{2}{*}{$\begin{array}{c}\text { FSL } \\
\text { tone (ms) }\end{array}$} & \multirow{2}{*}{$\begin{array}{c}\text { FSL } \\
\text { click (ms) }\end{array}$} \\
\hline & Phase-locked & PL-N & PL-MN & & & & & & \\
\hline 0.4 & $x$ & & & & & 4.9 & 133 & 7.3 & 4.0 \\
\hline 0.72 & $x$ & & & & & 9 & 333 & 7.4 & 6.6 \\
\hline 0.86 & $x$ & & & & & 0.6 & 96 & 6.8 & 5.2 \\
\hline 0.913 & $x$ & & & & & 14 & 157 & 6.7 & 6.0 \\
\hline 1.05 & $x$ & & & & & 18.5 & 308 & 5.1 & 4.1 \\
\hline 1.4 & & & & $\mathrm{x}$ & & 15.3 & 281 & 7.0 & 0 \\
\hline 2.37 & $x$ & & & & & 41 & 251 & 6.1 & 5.3 \\
\hline 3.56 & & $x$ & & & & 27.4 & 125 & 6.2 & 0 \\
\hline 6.4 & & $x$ & & & & 19 & 156 & 5.0 & 4.1 \\
\hline 7.3 & & $x$ & & & & 44 & 274 & 5.6 & 0 \\
\hline 7.7 & & & $x$ & & & 11.9 & 222 & 5.8 & 4.5 \\
\hline 8.0 & & & & $x$ & & 0 & 280 & 6.2 & 4.7 \\
\hline 8.2 & & $x$ & & & & 54.7 & 274 & 6.0 & 4.9 \\
\hline 12 & & $\mathrm{x}$ & & & & 82 & 241 & 5.6 & 4.9 \\
\hline 17.5 & & $\mathrm{x}$ & & & & 22.7 & 228 & 5.6 & 0 \\
\hline 17.6 & & & & & $x$ & 0 & 381 & 5.8 & 4.5 \\
\hline 18.9 & & & $x$ & & & 3.6 & 127 & 5.6 & 4.6 \\
\hline 24 & & $x$ & & & & 111 & 355 & 5.6 & 5.1 \\
\hline 35 & & $x$ & & & & 35 & 140 & 4.9 & 4.4 \\
\hline
\end{tabular}

Table 4-1.

Response characteristics of MNTB cells. Characteristic frequency (CF), primary-like with notch (PL-N), primary-like with multiple notches (PL-MN), chopper sustained (Chop-S), chopper transient (Chop-T), first spike latency (FSL). 
A

First spike latency to tone

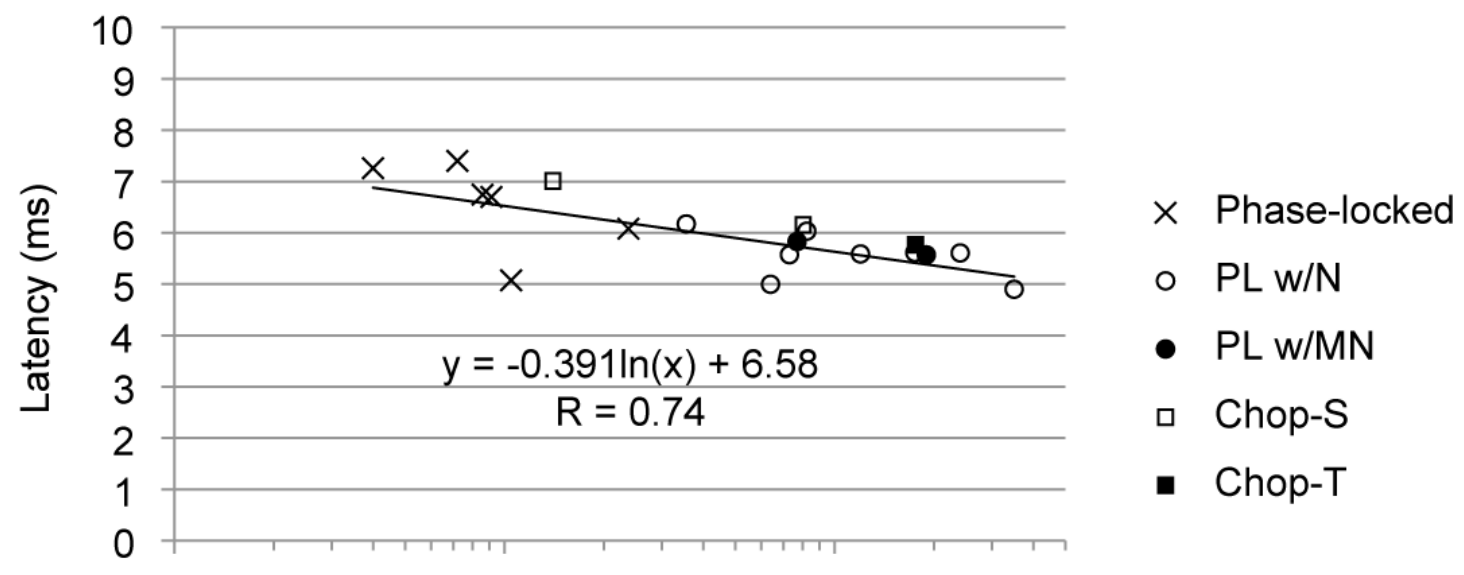

$\begin{array}{lll}0.1 & 1 & 10\end{array}$

Characteristic frequency $(\mathrm{kHz})$

B

First spike latency to click

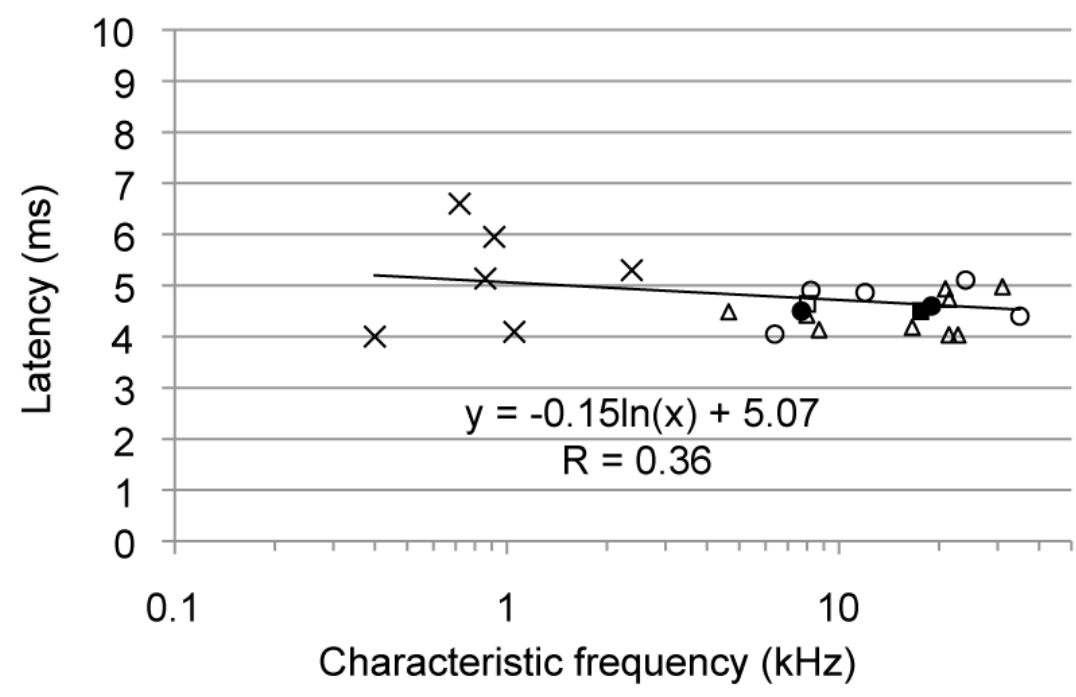

$\times$ Phase-locked

- PL w/N

- $P L W / M N$

- Chop-S

- Chop-T

$\Delta$ click only

Figure 4-7.

First spike latency for tone and click stimuli. First spike latency of 19 units for tone (panel A) organized by stimulus response, FSL average $6.0 \pm 0.73 \mathrm{~ms}$. There is no statistical correlations between CF and latency. FSL to clicks were shorter than FSL to tones and averaged $4.63 \pm 0.61$. Trend lines were fit to these data (equations in panels). 
A

Spontaneous rate

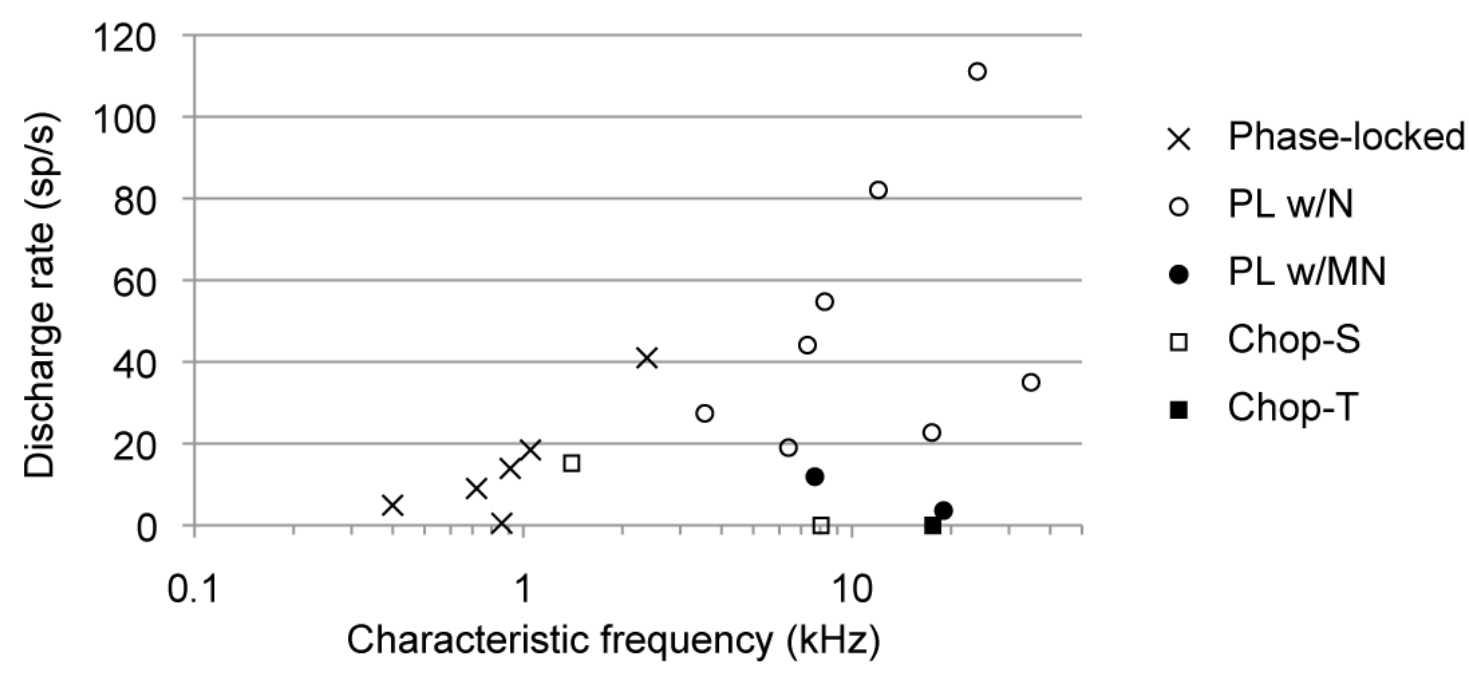

B

Max sustained discharge rate

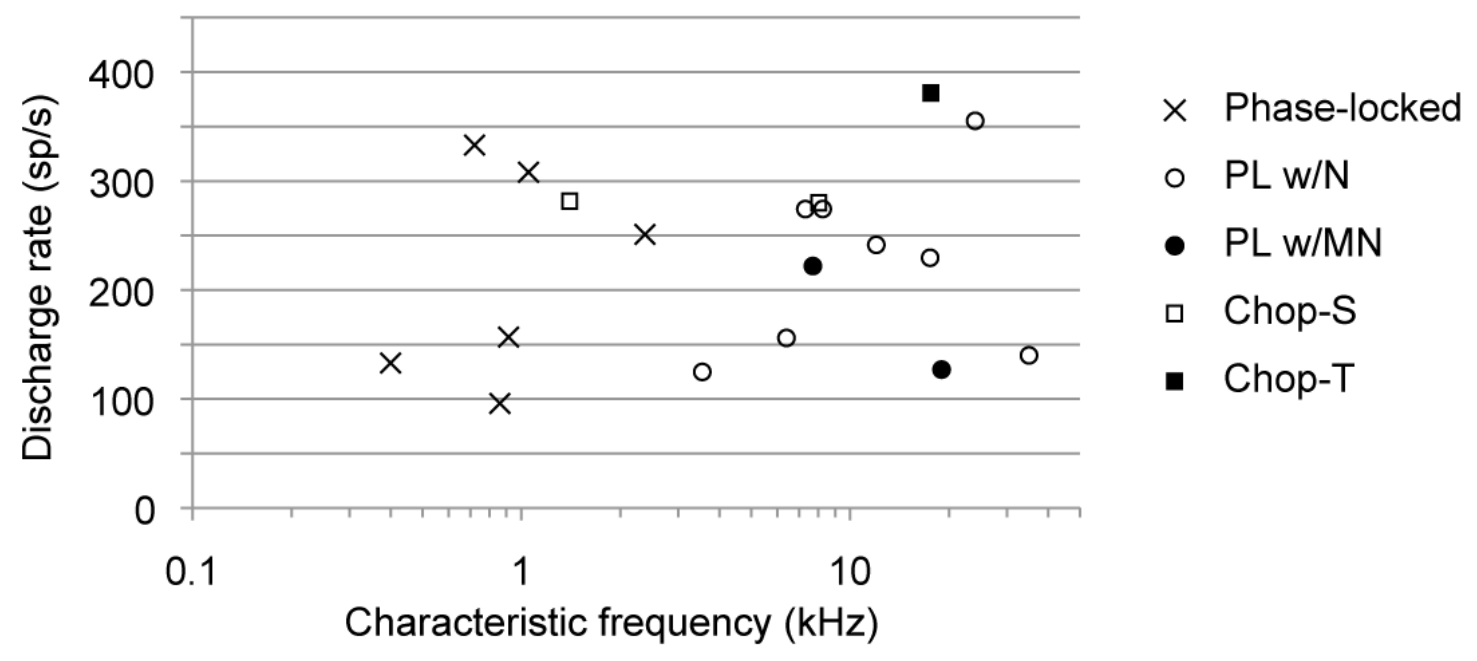

Figure 4-8.

Spontaneous and max sustained discharge rates. Spontaneous discharge rates (panel A) varied from near zero to over $100 \mathrm{sp} / \mathrm{s}$. Max sustained discharge rates averaged $200 \mathrm{sp} / \mathrm{s}$ (panel B). Discharge rates for phase locking units did not approach max firing rate observed in vitro of $500-800 \mathrm{~Hz}$. 


\section{A}

\section{Phase-locking $500 \mathrm{~Hz}$}
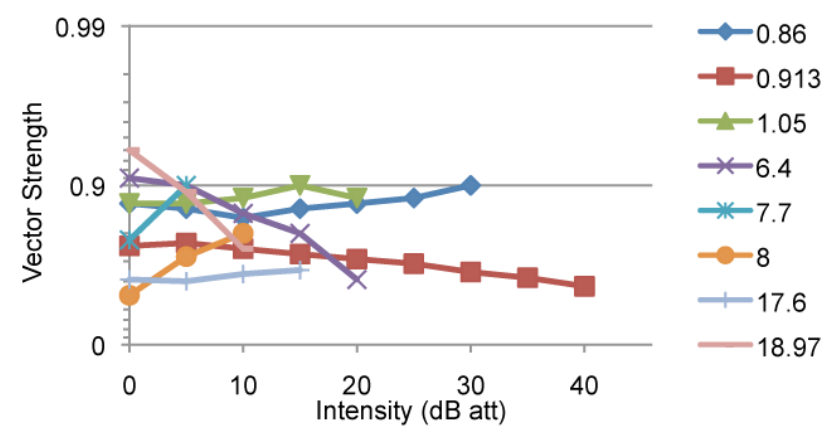

B

U9MAR2409n500I

$\mathrm{CF}=0.86 \mathrm{kHz}$
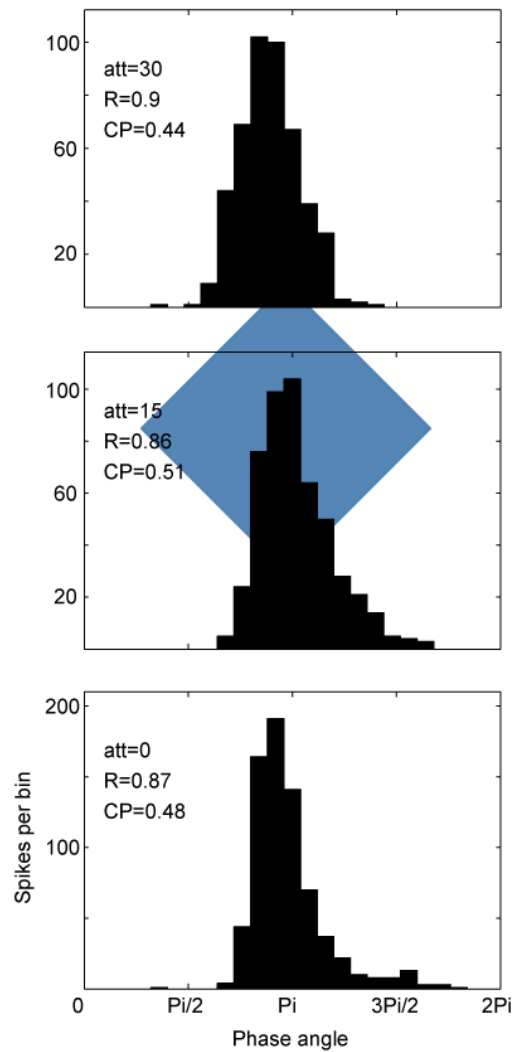

C

U12MAR2409n500I

$\mathrm{CF}=6.4 \mathrm{kHz}$
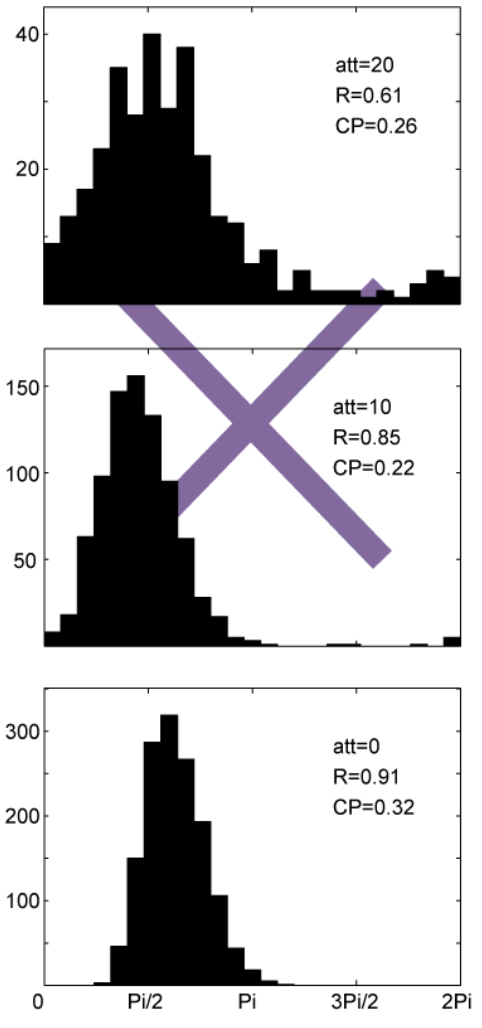

D

U5MAY3007n500I

$\mathrm{CF}=8 \mathrm{kHz}$
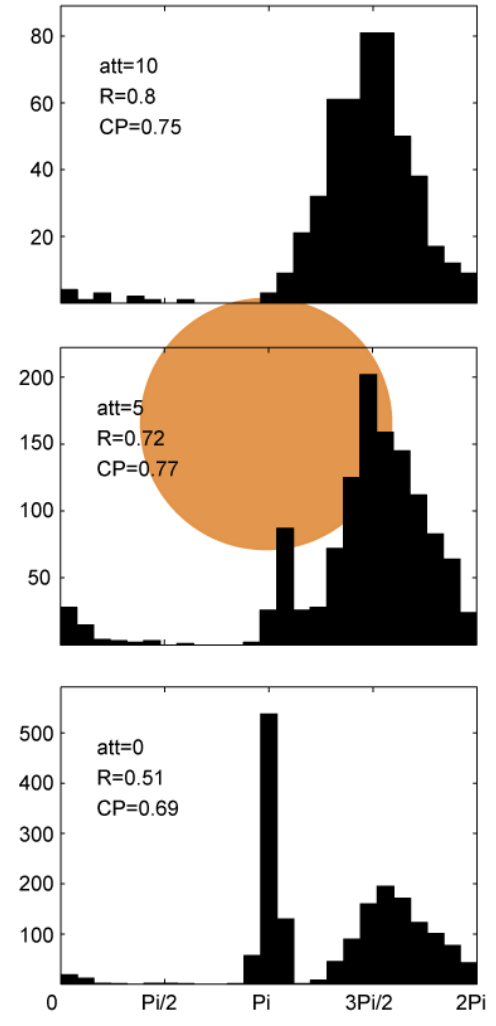

Figure 4-9.

Phase locking to $500 \mathrm{~Hz}$ pure tone. In panel A, vector strength (VS) measured from $500 \mathrm{~Hz}$ stimuli across intensities is shown for several low and high CF units. In panels $B, C$ and $D$ phase PSTHs are shown for several stimuli intensities. Vector strengths could remain high across intensities (blue squares, green triangles, light blue crosses and column B). In some units VS increases with intensity (pink dashes, purple exes, burgundy squares and column C) but in others VS decreases (orange circles, blue exes and column D). 


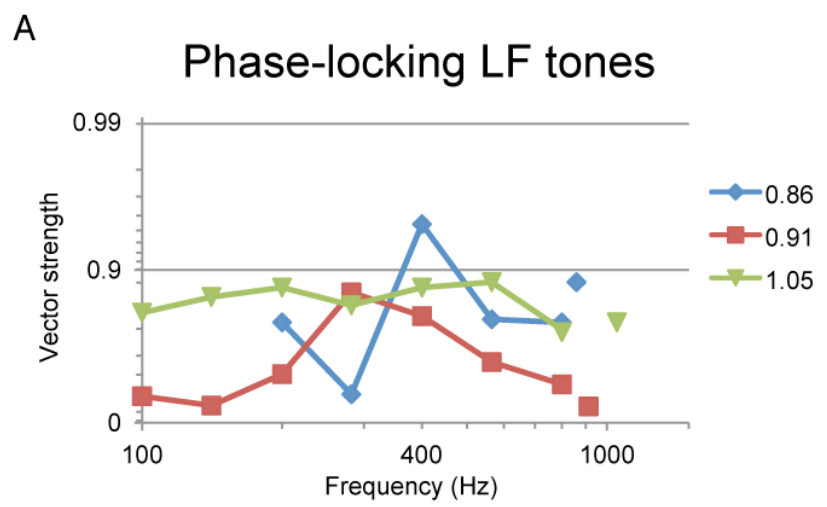

B

U9MAR2409n500I

$\mathrm{CF}=0.86 \mathrm{kHz}$
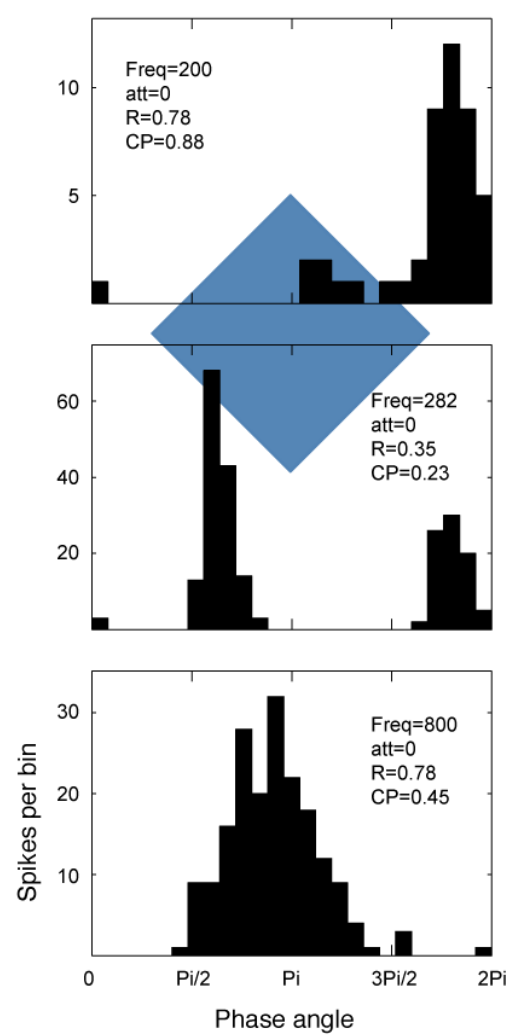

C

\section{U19DEC1306nphasel}

$\mathrm{CF}=0.91 \mathrm{kHz}$
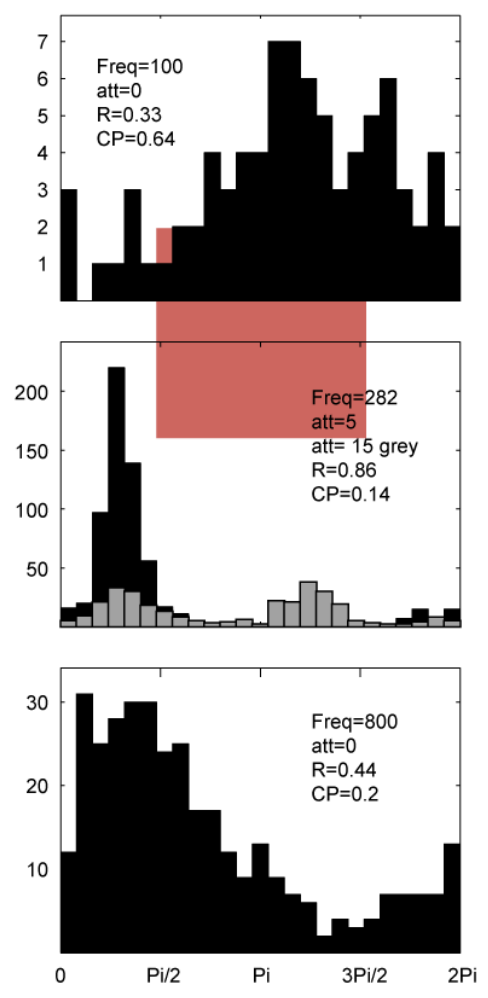

D

U4JAN1007nphasel

$\mathrm{CF}=1.05 \mathrm{kHz}$
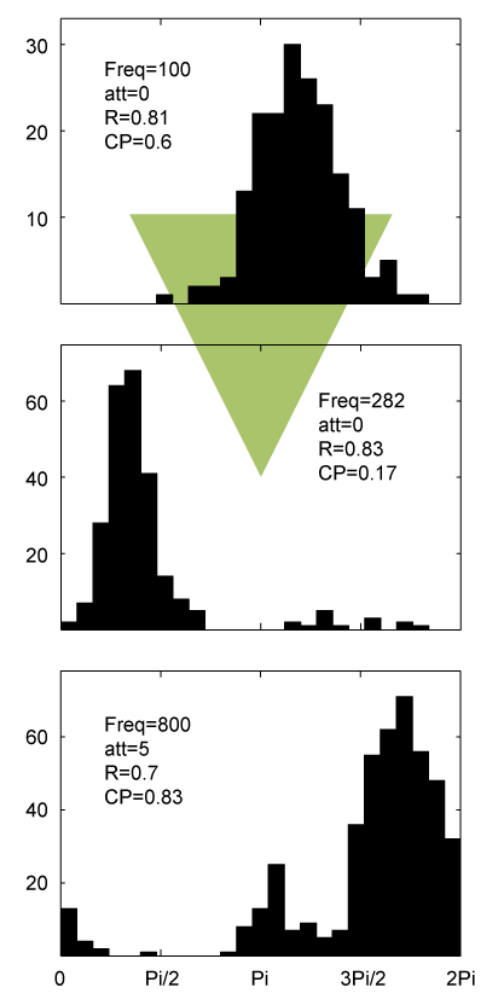

Figure 4-10.

Phase-locking in low CF MNTB units. Vector strength (VS) varies as a function of stimulus frequencies in our low CF population (panel A). We found that frequency sensitivity and peak splitting were the two most dominant factors that contributed to VS (columns B, C and D). In column B, VS remained strong (>.78) across frequencies except for where the peak splits. CF did not necessarily correlate with highest VS (column $\mathrm{C}$ ) and this unit (CF=0.91 kHz) was especially sensitive to intensity at some frequencies. For this cell, when the stimuli $(\mathrm{f}=282 \mathrm{~Hz})$ 
intensity was close to threshold the phase PSTH was split (grey bars, column C row 2) but at higher stimulus intensities phase-locking was unified and VS high (VS=.86 black bars). In the last column VS strength remained high across all stimulus frequencies. 
A

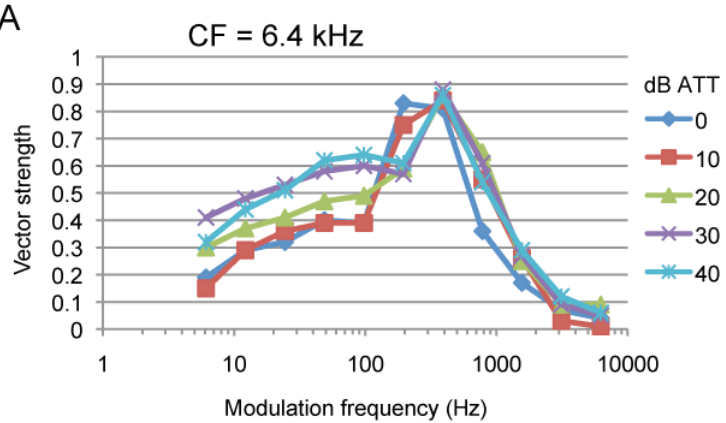

C

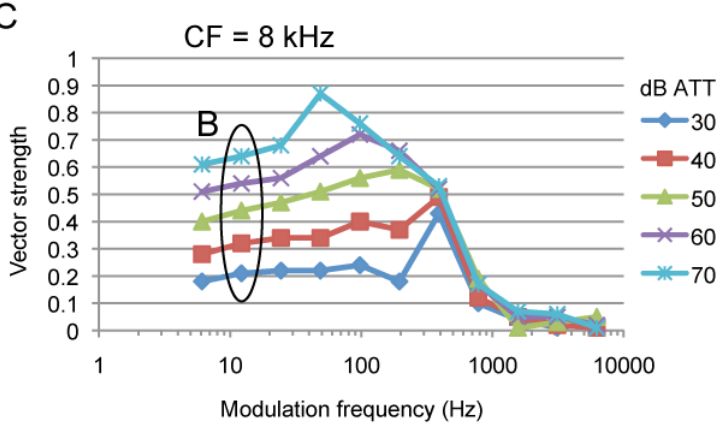

E

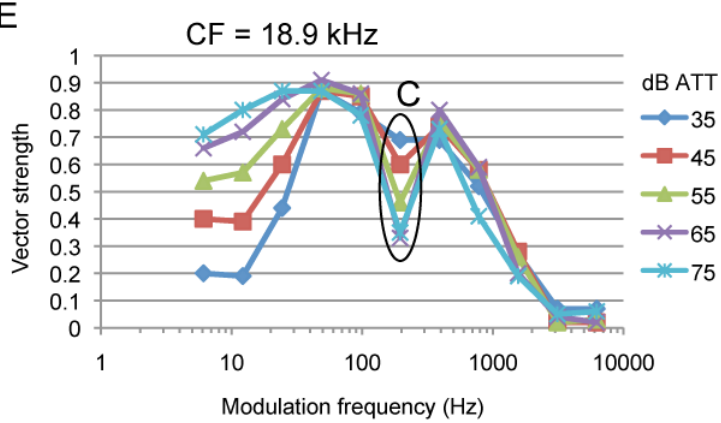

B

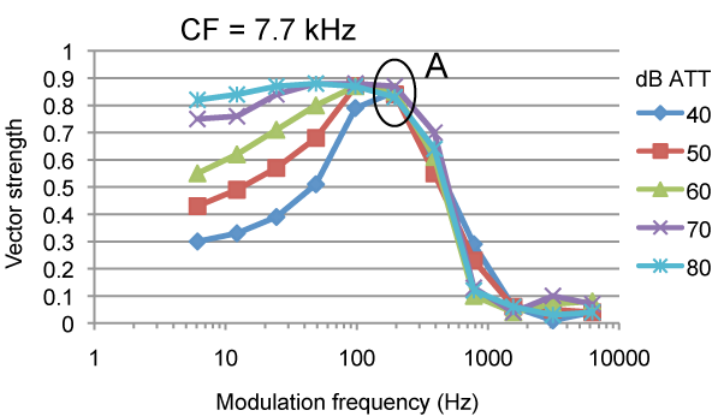

D

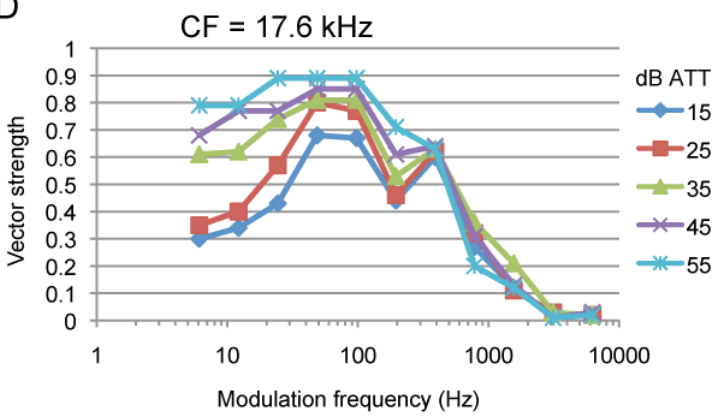

F

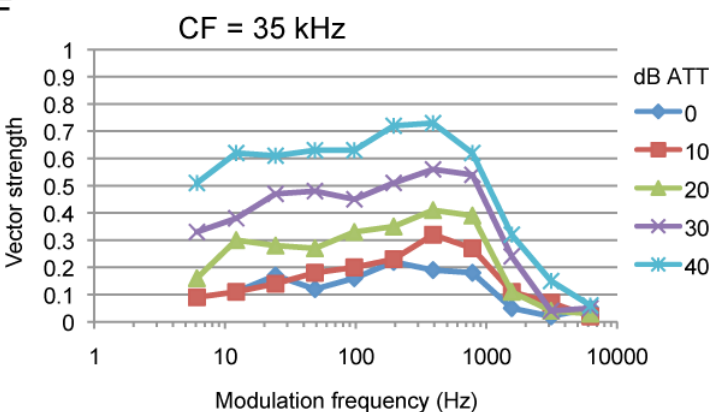

Figure 4-11.

Temporal modulation transfer functions (tMTFs). Temporal modulation transfer functions, for units localized to the MNTB shown in the PSTH figures, demonstrate that neuron sensitivity to modulation of the CF, as expressed by vector strength, were more or less sensitive to intensity of the stimuli (in dB ATT). The population was too small to allow us to draw any conclusions from CF to tMTF shape as the 2 units most insensitive to intensity had CFs at the opposite end of our MNTB population, panels A and F. Almost all cells had a modulation frequency at which intensity had little to know effect on VS (characteristic modulation frequency $(\mathrm{cMF})=400 \mathrm{~Hz}$ for panels $\mathrm{A}, \mathrm{C}, \mathrm{D}, \mathrm{E}$ and $\mathrm{cMF}=200 \mathrm{~Hz}$ for panel $\mathrm{B}$ of cells Panels A-F show sensitivity to SAM were sensitive to intensities to varying degrees having VS for SAM tones in band pass and low pass filter shapes. Exceptions to intensity sensitivity are shown in Panels A and F. Panel A TMRF is that of a typical band pass filter 
A

\section{U11MAY3007naml} mod freq $=195.31$
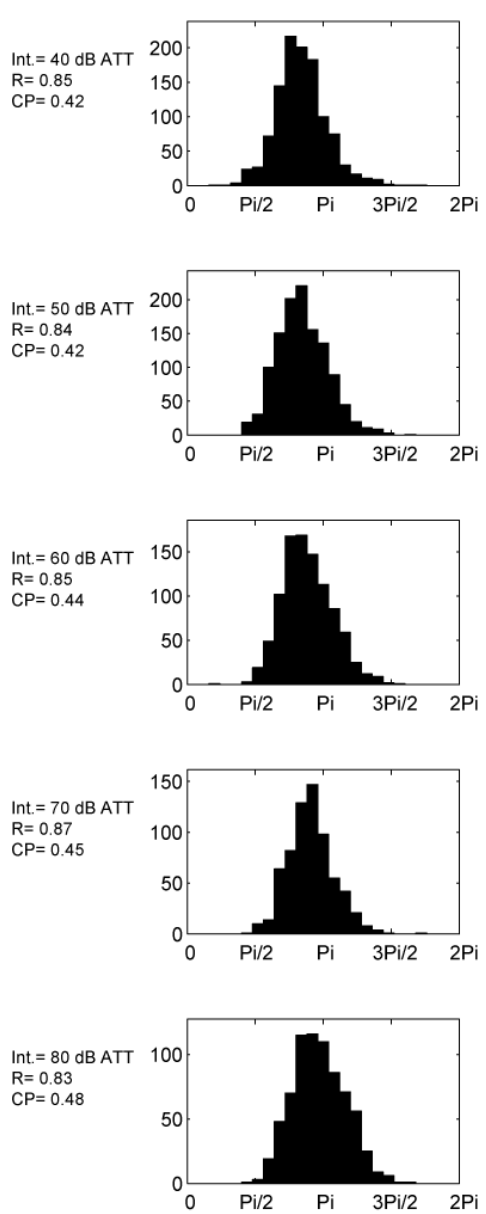

B

U5MAY3007naml

mod freq $=12.21 \mathrm{~Hz}$
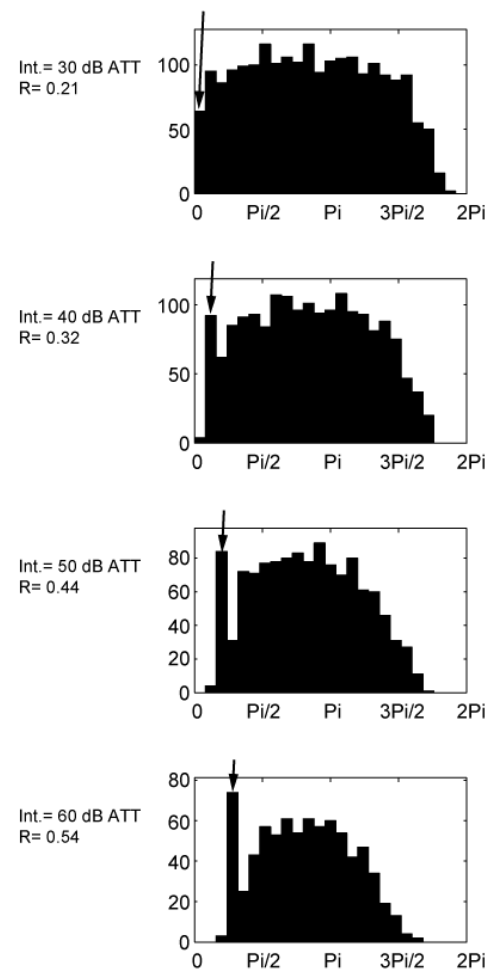

Int $=70 \mathrm{~dB}$ ATT $R=0.64$

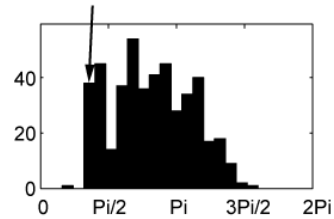

C

U4MAY3007naml

mod freq $=195.3125$
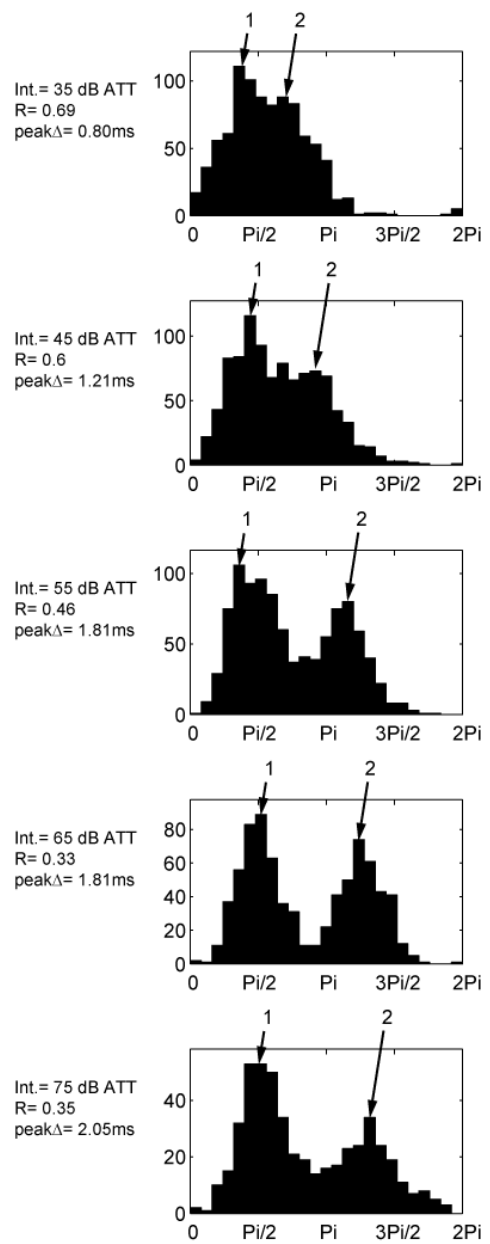

Figure 4-12.

Phase PSTHs for SAM tones. Intensity dependence of vector strength is shown in the phase PSTHs of 3 units. Certain modulation frequencies were unaffected by intensity (column A) and while VS were maintained phase PSTHs often changed shape going from Gaussian to Poisson-like or sometimes developing a low discharge rate tail at higher frequencies.

Common among all responses, was a shift in the phase of the first responses of a cell. This effect was more pronounced at lower frequencies, compare column B with A. 
5. Discussion 


\section{Methodology: Localization of Single Unit Recordings to the MNTB}

We used a dorsal approach to the MNTB, which entailed penetration through distances greater than $6 \mathrm{~mm}$ to reach the dorsal aspect of auditory brainstem cell groups. Therefore, the process of aligning the electrode relative to brain coordinates needed to be accurate and reproducible. Given small variation in brain size and position of cell groups, we made electrode tracks according to a grid applied to the brainstem surface, whereby penetrations moved along a medial-lateral row at $0.2-0.5 \mathrm{~mm}$ increments, and mediallateral rows were placed at $0.5 \mathrm{~mm}$ rostro-caudal increments. Selected electrode tracks were recovered based upon tract tracer or lesion placements, and other electrode tracks were mapped to the tissue relative to these landmarks. By reconstructing tracks in 3D, we could localize most recording sites not only to the MNTB, but also to other cell groups of the SOC (not reported here). Glass pipette electrodes were used for all recordings and to iontophorese BDA. These pipettes were tailored to produce small tract tracer injection sites and yet have the capability of recording single units. They had diameters of 2-4 $\mu \mathrm{m}$ and impedances of about $10 \mathrm{M} \Omega$. Based upon experience (Spirou et al. 1990), it is difficult to isolate large diameter axons of the cat trapezoid body for more than several minutes using electrode tips with these characteristics. However, for consistency in recording parameters and simplicity in using the same amplifier throughout the experiment, we used glass pipettes for all recordings. In multiple published extracellular single unit studies, prepotentials, which are a variable amplitude wave preceding the action potential, have been used to identify principal neurons of the MNTB. Prepotentials are more difficult to isolate with glass than with metal electrodes, and our recording system was not capable of averaging more than $300 \mu$ s prior to a level threshold used to detect action potentials. Therefore, we did not rely upon prepontential detection to distinguish MNTB neurons from local or traversing nerve fibers. We did apply a strict criterion of requiring at least a 20-minute holding time to classify a recording as occurring from a neuronal cell body, which likely excluded many bona fide cellular recordings from our database. Decerebrate preparations typically maintain higher blood pressure than anesthetized animals, and the attendant regular small pulsations due to heart rate are difficult to control with the pneumothorax procedure. Therefore, we are confident that the recordings reported here are from neuronal cell bodies located within the confines of the MNTB. Note, however, that we cannot distinguish principal from non-principal cell recordings, 
the latter of which constitute a small percentage of cells in cats (Morest, 1968; Spangler et al., 1985).

\section{Driven Activity in the MNTB}

The security to which the calyx of Held faithfully elicits an action potential from MNTB cells is important to understanding the role of the MNTB in ITD and ILD processing. Intense interest in this topic was generated by a report, in gerbil, that failures were quite common in vivo (Kopp-Scheinpflug et al., 2003a). Subsequent investigation, also in gerbil, revealed failure in vitro but at a much lower rate (Hermann et al., 2007). The report by KoppScheinpflug et al. (2003a) challenged the dogma of secure, faithful synaptic connection. This dogma was based upon in vivo recordings in cat, that failures of the calyx of Held only occurred when GBC axons were electrically stimulated at frequencies greater than $500 \mathrm{~Hz}$ and no failures were observed in these cells when calyx discharge was driven via sound stimulation (Guinan and Li, 1990). Recent supporting evidence, also from cat in vivo, indicated that failure of the calyx to induce MNTB AP generation is very rare, leading to the suggestion that evidence of calyx failure in previous in vivo studies is the result of the electrode placement near calyces not connected to the cell of interest (Mc Laughlin et al., 2008) and possibly species differences. By comparing the discharge rates of GBCs and large trapezoid body fibers to discharge rates of MNTB cells, a clearer picture of synaptic integrity of the calyx of Held can be gained. In anesthetized cats, low frequency GBC and trapezoid body fibers (TB) can entrain (discharge every cycle) to the phase of the stimulus (CFs $\leq 600$ $\mathrm{Hz}$ ) and consequently sustained discharge rates have been measured up to $600 \mathrm{sp} / \mathrm{s}$ (Joris et al., 1994b). Joris showed that sustained discharge rates were lower ( $\max =400 \mathrm{sp} / \mathrm{s})$ in phase-locked units that could not entrain, which are those having CFs greater than $600 \mathrm{~Hz}$. Other studies of TBs report more modest sustained discharge rates for low CF units. Spirou et al. (1990) recorded from low CF $(<2 \mathrm{kHz})$ phase-locked trapezoid body axons that did not demonstrate perfect entrainment and had maximum sustained discharge rates below 500 $\mathrm{sp} / \mathrm{s}$. Brownell reported that axons of presumed GBCs in the trapezoid body generate a maximum sustained discharge averaging $252 \pm 19.7 \mathrm{sp} / \mathrm{s}$ (mean $\pm \mathrm{SEM}, \mathrm{CF}=0.6-30 \mathrm{kHz}$ ). In the unanesthetized decerebrate cat, our recordings of MNTB cells demonstrated a maximum sustained discharge rate of $381 \mathrm{sp} / \mathrm{s}$. Phase-locked units had sustained discharge 
rates up to $333 \mathrm{sp} / \mathrm{s}$. Our lowest $\mathrm{CF}$ unit $(\mathrm{CF}=400 \mathrm{~Hz})$ did demonstrate entrainment to the stimulus and had a maximum sustained discharge rate less than $200 \mathrm{sp} / \mathrm{s}$. Our measures of maximum sustained discharge rate of MNTB cells in decerebrate cat are similar to those reported in trapezoid body fibers by Brownell (1975), but were less than measures of sustained discharge rates reported in trapezoid body fibers by Spirou et al. (1990) and in GBCs by Joris et al. (1994b). It is difficult to reconcile these differences (lack of entrainment in units having $\mathrm{CF}<600 \mathrm{~Hz}$ and an overall lower maximum sustained discharge rate than are found in most other studies), as the general effects of barbiturate anesthetics act as an agonist on GABA receptors (Olsen and Guo-Dong, 2011). Lowered maximum sustained discharge rates might result from GBCs projecting to the MNTB. Between 9-69 excitatory modified end-bulbs of held from auditory nerve fibers can be found on the cell bodies of GBCs. In addition to these excitatory inputs, inhibitory inputs can be found on the soma and occupy $3-10 \%$ of the somatal surface (Spirou et al., 2005). Pharmacological blockade of these inhibitory inputs in gerbils (Kopp-Scheinpflug et al., 2002) increases maximum sustained discharge rates and therefore rules out synaptic circuitry innervating GBCs as a cause of decreased output from MNTB cells. Immunohistochemistry studies show that inhibitory inputs found on MNTB cells can either be glycine or GABA immunoreactive (gerbil, Roberts and Ribak, 1987; cat, Adams and Mugnaini, 1990; Spirou and Berrebi, 1997). If the circuitry were simple and these GABAergic inputs were driven mainly by excitation, then maximum sustained discharge rates would be higher in the unanesthetized prep than in the anesthetized animal. One mechanism for barbiturate anesthesia to enhance maximum discharge rate relative to the unanesthetized, decerebrate condition, might be through the GABAergic inhibition of glycinergic inputs on MNTB cells. The most likely candidate of GABA controlled glycinergic inhibition on MNTB cells is other MNTB cells (Kuwada and Zook, 1991; Smith et al., 1998). While other sources of these inhibitory inputs have been proposed, collaterals branching from MNTB neurons projecting to the MSO and LSO are the most elegant solution. The use of non-barbiturate anesthetics in gerbils resulted in maximum sustained discharge rates (approximately 300sp/s) similar to those we report (KoppScheinpflug et al., 2008). Most experiments in the brainstem last hours and consequently the level of anesthesia varies throughout the experiment as maintenance doses are given. While spontaneous rates are not comparable to driven rates, as the level of excitation and inhibition 
varies with stimulus and intensity, Kopp-Scheinpflug et al. found that in the MNTB spontaneous rates did not vary with the level of anesthesia (in rats, gerbils and mice) or type of anesthesia (barbiturate vs. non-barbiturate in rats).

\section{Low frequency tuning in the MNTB}

In our dorsal approach to the MNTB we sampled cells that had CFs, which ranged from $0.4-35 \mathrm{kHz}$, and these were consistent with ranges reported in previous studies of the cat MNTB (0.3 - 28 kHz, Guinan et al., 1972b; 0.9 - 30 kHz, Tsuchitani, 1994, 1997; $0.4-35$ kHz, Smith et al., 1998). Tonotopic organization of the SOC, reported by Guinan et al. (1972), demonstrated that while the entire frequency range is represented in the MNTB, MSO and LSO, the MNTB and LSO had tonotopic organization that emphasized higher frequency ranges. In recordings by Guinan et al. (1972 a,b) from MNTB, 17/32 (54\%) of recorded neurons had CFs greater than $5 \mathrm{kHz}$. Low and mid frequency regions were roughly equivalently represented, containing $21 \%$ and $25 \%$ of neurons in the MNTB respectively. In our population of MNTB cells, albeit small, $30 \%$ (6 of 19) had CFs $<2 \mathrm{kHz}, 10 \%$ had CFs of $2-5 \mathrm{kHz}$ and over $50 \%$ had CFs $>5 \mathrm{kHz}$. To access the SOC, Guinan et al. (1972 a,b) utilized both dorsal and ventral approaches. Ventral approaches to the MNTB performed by Tsuchitani (1997) and Smith et al. (1998) yielded far lower percentages of low (<2 kHz) CF units, 11\% ( $n=64)$ and 5\% $(n=45)$ respectively. These studies (Tsuchitani, 1997; Smith et al., 1998) did not examine the distribution of CF specifically and it is difficult to estimate the mid and high frequency populations from the data presented in the papers. However, Smith et al. (1998) indicated that CFs of the MNTB population presented might be skewed as the ventral approach to the MNTB precludes easy access to low frequency MNTB cells.

\section{PSTH properties of MNTB cells}

Because reports of the physiological characteristics of MNTB units in cats are few (Guinan et al., 1972a; Tsuchitani, 1994; Tsuchitani, 1997; Smith et al., 1998), it is necessary to compare our results to GBC and MNTB cells recorded in cats and other species. Reports of physiologically characterized MNTB neurons in cats (Guinan et al.,1972a,b; Guinan and Li, 1990; Smith et al., 1998; Tsuchitani, 1994, 1997) were all performed in anesthetized animals. Our experiments were performed in decerebrate unanesthetized animals, which differ in the 
activation of inhibitory inputs, and we were interested in how responses may differ in comparision to the anesthestized state.

As described in a previous section, debate continues over the role of MNTB as a faithful signal inverter, converting precisely timed/phase-locked excitatory input from GBCs of the VCN into precisely timed/phase-locked inhibitory input to the MSO and LSO. While the main input to each MNTB cell is a single large excitatory calyx of Held, many inhibitory and excitatory non-calcyeal inputs exist on the soma (Roberts and Ribak, 1987; Adams and Mugnaini, 1990; Spirou and Berrebi, 1997). Recordings of GBCs in the cochlear nucleus that combined intracellular recording with cellular labeling show that these cells respond to pure tone stimuli with phase-locked responses for low CF cells, and $\mathrm{PL}_{N}$ discharge patterns for cells having higher CFs (Rhode et al., 1983; Rouiller and Ryugo, 1984). Extracellular recordings in the AVCN have shown that units classified as $P L, P L_{N}$, phase-locked, and onset often exhibit prepotentials (a physiological characteristic of SBCs and GBCs) in spike voltage waveforms (Young et al., 1988; Blackburn and Sachs, 1989). Not all units verified as GBC demonstrate prepotentials in individual waveforms and often it is necessary to average spike waveforms to visualize prepotentials (Bourk, 1976; Young et al., 1988). GBCs are found in areas, specifically the VCN and auditory nerve root area (Osen 1969; Tolbert and Morest 1982), that also demonstrate Chop-S and Chop-T discharge patterns (Young et al., 1988). The ventral component of the trapezoid body, consisting of axons originating from GBCs (van Noort, 1969; Brownell, 1975), demonstrate $P L, P_{N}$ and onset-L responses and thus provide additional evidence that MNTB cells receive non- $\mathrm{PL}_{\mathrm{N}}$ excitatory inputs via the calyx of Held (Spirou et al., 1990). Rhode (2008) recently confirmed, via intracellular recordings, that GBC response patterns are more varied, exhibiting not only Phase-locked, $\mathrm{PL}_{N}$ and $O n-\mathrm{L}$ discharge patterns but at lower stimulus intensities, PL as well. In agreement with other studies, we found that MNTB units have phase-locked and $\mathrm{PL}_{N}$ responses to pure tones (Smith et al., 1998; Tsuchitani, 1994,1997; gerbil, Kopp-scheinpflug et al., 2003); however, we found additional response patterns in small numbers (Chop-S, Chop-T and $P L_{M N}$ ) consistent with the most thorough sampling of MNTB response types (Guinan et al., 1972a). As we did not select for prepotentials, MNTB cells reported could arise from non-principal cells, which comprise $\sim 5 \%$ of the MNTB cell population, so could potentially account for the three chopper cells in our sample. The role of inhibitory inputs found on MNTB cells in the 
shaping of PSTHs is unclear and response types that we recorded in MNTB cells are likely demonstrated in GBCs in unanesthetized preps (see Spirou et al., 2005 for a discussion of inhibitory inputs effects on GBC output).

MNTB first spike latencies suggest that inhibition arrives before excitation to the MSO

The peak of the ITD curve occurs at positive values for most MSO cells. This observation has been attributed to inhibition from the MNTB reaching MSO cells before excitation from contralateral spherical bushy cells (Brand et al. 2003). Our original goal was to test this notion by measuring first spike latencies of MNTB and MSO cells in response to contralateral sound of sufficient loudness to activate MSO cells monaurally. However, our MSO cell population was too small to make these comparisons (not presented here). We do report FSL measurements from MNTB cells as a baseline for future studies of MSO. In this section, our data are compared to other studies in cats. Recordings in the trapezoid body of axons originating from GBC's and SBC's revealed a population with FSL to clicks ranging from over $5 \mathrm{~ms}$ (from low CF fibers) to less than $2 \mathrm{~ms}$ (high CF fibers) (Brownell, 1975; Guinan et al. 1972). In the MNTB, Guinan et al. found click FSL of 4-5 ms for cells having $\mathrm{CFs}$ less than $2 \mathrm{kHz}$. Cells having CFs greater than $2 \mathrm{kHz}$ had first spike latencies in the 2-4 ms range (Guinan et al., 1972). In our data, FSL to click for low $(<2 \mathrm{kHz}) \mathrm{CF}$ units averaged $5.16 \pm 1.14 \mathrm{~ms}$ and for high CF $4.55 \pm 0.39 \mathrm{~ms}$. Differences in FSLs we measured and previous reports are likely due to differences in click characteristics such as width and intensity.

First spike latencies (FSLs) to tones are sensitive to the duration of the onset ramp (Tsuchitani, 1997), and can vary as much as $2.7 \mathrm{~ms}$ with a $1.5 \mathrm{~ms}$ difference in onset duration. The gating characteristic of our pure tones ( $2 \mathrm{~ms}$ ramp, see methods) fell between onset ramps used by Smith et al. (1 ms ramps, 1998) and within the range used by Tsuchitani (1,2 and $2.5 \mathrm{~ms}$ ramps, 1997). The FSL we report for $\mathrm{CF}>3 \mathrm{kHz}$ are in general agreement with those reported by Smith et al. (1998) and Tsuchitani (1997). However, Tsuchitani (1997) did not record from cells with $\mathrm{CF}<3 \mathrm{kHz}$ and Smith et al. (1998) recorded from only 2 cells having $\mathrm{CF}<3 \mathrm{kHz}$. We found $\mathrm{FSL}$ in our population of cells ( $\mathrm{CF}>3 \mathrm{kHz}$ ) was more uniform than FSL reported by Tsuchitani (1997) for tones having $2 \mathrm{~ms}$ onset ramps. 
Phase-Locking of MNTB units compared to their inputs

Many GBCs phase-lock with higher VS (termed enhanced phase-locking) to low frequency tones than do ANFs (Joris et al., 1994b). While high CF GBCs do not phase-lock at CF, their VS in response to low frequency stimuli can exceed VS of low CF units (Joris et al., 1994b; Joris et al., 1994a). We measured phase-locking of low CF MNTB cells to a range of low frequency stimuli across intensities and found that although these cells phase-locked to all of the low frequency stimuli presented we did not observe the enhanced phase-locking $(>0.95)$ found in GBCs (Joris et al., 1994 a,b). High CF units were capable of phase locking to low frequency stimuli, but VS was similar to values recorded from low CF cells. If our small sample is representative of MNTB responses, then the less precise phase-locking we observed is likely due to modulations in synaptic integration. Coefficient of variation (not reported) of first spike latency was consistent with other reports (Tuschitani, 1997; Smith et al., 1998) indicating that the observed low VS, compared to GBCs, was due to exhaustion of the calyx (Kopp-Scheinpflug et al.) or through additional synaptic mechanisms uninhibited in the decerebrate preparation.

\section{Phase-Locking to SAM tones}

To examine temporal coding of high frequency sounds we utilized SAM tones. Because high frequency tones are transduced into a signal containing no phase information it has been postulated that high CF MSO cells utilize the temporal envelope of high frequency stimulus to code ITDs. We presented SAM tones to MNTB units having CFs $>5 \mathrm{kHz}$ at a broader range of modulation frequencies $(6.1-6250 \mathrm{~Hz})$ than is typically presented in studies of SAM tones. The phase-locking to SAM tones was quantified as the VS, and plots of VS across modulation frequency, termed temporal modulation transfer functions (tMTF), were generated. We focused sequentially on the low modulation frequency, peak, and high modulation frequency rolloff responses. Six cells were studied using the complete protocol for SAM tones; VS were calculated and tMTFs were generated for comparison to other studies.

While phase-locking to SAM tones has been investigated in the context of ILD coding (Joris and Yin, 1998) only recently has temporal coding to SAM tones been investigated more thoroughly in MNTB of gerbils (Kopp-Scheinpflug). However, these studies are limited in the data that they present as described below. We found that at low MF, vector strength 
decreased with increasing stimulus intensity; however, at higher MF, VS increased with increasing intensity, having a peak VS between 100-400 MF (Hz). Because of these changes with intensity, tMTFs were initially low pass in shape but became band pass at higher stimulus intensities. In the ILD circuitry of the cat, Joris and Yin (1998) showed that SBCs, GBCs and MNTB cells all demonstrate low-pass tMTFs. Phase-locking was reported in modulation gain and not VS so direct comparisons are difficult. Joris and Yin (1998) showed maximum phase locking values for their MNTB population that varied from $0.6-0.9$. Phaselocking of MNTB cells to SAM tones in our population was strong and maximum VS varied between 0.8 and 0.9. The exception ( $\mathrm{CF}=35 \mathrm{kHz}$ ) is in agreement with Joris and Yin (1998) that high (>20 kHz) CF cells demonstrate poor phase-locking to SAM tones. These authors did not show intensity effects for MNTB cells; however, phase-locking of GBCs decreased uniformly across MF as intensity increased (Joris and Yin, 1998). The MNTB of rats had low pass tMTFs, demonstrated weaker phase-locking (max average VS $=0.6$ ) across MFs and lower VS at higher MFs compared to cat (Kadner and Berrebi, 2008; Tolnai et al., 2008). Our phase PSTHs often demonstrated multiple peaks within the length of a waveform that were more evident in SAC analysis (data not shown). Most studies do not report or show these data; however, one report of SAM responses of cells in the DCN and PVCN in unanesthetized cat shows that through fourier analysis of phase PSTHs second harmonic components of the modulation frequency were revealed (Kim et al., 1990). These complex responses and enhanced phase-locking to SAM tones that we observed in the MNTB are likely the consequence of an intact inhibitory system of an animal that specializes in low frequency sound localization. 


\section{Conclusion}


Physiological response properties of MNTB neurons presented in Chapter 4, together with anatomical findings in Chapter 3, have implications on how future computational models should address inhibition in ITD coding circuitry of the SOC. Further investigation into the sound localization of low frequency stimuli is warranted in larger headed mammals because doubt exists as to whether rate or place (or both) coding strategies are used to locate low frequency sounds in space. Rate coding of azimuthal location in ITD sensitive rodents (guinea pigs and gerbils) is widely accepted and some mechanisms by which peak discharge rates are shifted, such as inhibition and axon location on soma or dendrites, are mostly understood.

In the discussion of MSO function, it becomes necessary to differentiate sound frequencies that generate phase-locked inputs ( $<\sim 4 \mathrm{kHz}$ in cat) from higher frequencies. The range of CFs represented in the cat MSO varies from $100 \mathrm{~Hz}$ to well over $10 \mathrm{kHz}$ (Guinan et al.,1972a,1972b). At frequencies $<4 \mathrm{kHz}, \mathrm{MSO}$ and IC neurons encode the fine structure of acoustic waveforms and at frequencies $>4 \mathrm{kHz}$, they encode ITDs for the envelopes of sounds (rabbit, Batra et al., 1997; guinea pig, Griffin, 2005). The size of an animal's head further restricts the range of frequencies available for sound localization using ITD coding such that the interaural distance should be less than the wavelength of sound. For a cat, with an ecological range of $\pm 400 \mu \mathrm{s}$, we consider frequencies less than $2.5 \mathrm{kHz}$ to be the limit of ITD coding and examine these frequencies in the following discussion.

As head size increases, ecological ITD range increases and the audible spectrum extends to low frequencies in the tens of $\mathrm{Hz}$ range. Psychophysical studies in humans show that ITD sensitivity to low frequency sounds degrades below $100 \mathrm{~Hz}$; the upper limit is approximately $1.6 \mathrm{kHz}$ (Blauert, 1997). Phase-locking to low frequency stimuli $(\sim 100 \mathrm{~Hz})$ produces modulation in the discharge rate of cells projecting to the MSO over several ms, much longer than the ecological ITD window of humans ( $<800 \mu \mathrm{s})$. At frequencies greater than $1.2 \mathrm{kHz}$ the period of the stimulus is short and peak discharge rates can occur twice within the ecological window. Correspondingly, more than one wavelength of the sound will fit within the interaural distance, leading to ambiguity for encoding sound localization. ITD circuitry, then, must encode sound location across the useful range for frequencies. The prevailing mechanisms to accomplish this task, place and rate coding, each have their 
strengths and weaknesses across the ITD-sensitive frequency range. In this dissertation, we combined anatomical studies of ITD circuitry in cats and computational modeling studies based on a cat MSO neuron. As a result, much of the discussion considers utility of cellular mechanisms to encode ITD in animals with larger heads than the typical rodent models. In a model MSO cell, I showed that two sources of inhibition improved ITD coding relative to a single source of inhibition by narrowing the ITD curve and offering greater flexibility in shifting the peak of the ITD curve, and with efficacious combinations of IPSP timing could shift peak discharge rates to more positive delays. How two sources of inhibition affect ITD coding at frequencies greater than $250 \mathrm{~Hz}$ was not explored in the model, but have been elsewhere in a linear summation model (Leibold, 2010). Because we utilized a morphologically accurate MSO cell with the full complement of ion channels our results will guide the following discussion of neural coding at higher and lower frequencies.

In cats and dogs, animals with relatively large heads, physiological evidence supports a place code model (Goldberg and Brown, 1969; Yin and Chan, 1990). An essential feature of a place code is an array of cells with ITD peaks within the ecological range. The physiological evidence in large headed mammals, such as cat and dog, supports a place code as evident by the many MSO cells having a range of peak ITDs within the ecological window (Goldberg and Brown, 1969; Yin and Chan, 1990). The defining feature is an orderly distribution of best ITD with place within the MSO. In cats, a rough pattern of increasing peak ITD with rostro-caudal distance is the best evidence for a place code in the MSO (Yin and Chan, 1990). Our biophysical model offers the capability, using a second source of inhibition, to achieve a range of peak ITDs up to $400 \mu \mathrm{s}$, and so could support a place code model. Both Goldberg and Brown (1969) and Yin and Chan (1990) also reported physiological characteristics of cells that do not fit a place code, in that nearly half of the ITD sensitive MSO cells had peak discharge rates near or outside the ecological window for their respective animal models. These response properties fit better to a rate code strategy, and offer the possibility that the MSO in animals with large heads do not rely exclusively on a place or rate coding mechanism.

In animals with small heads (gerbils and guinea pigs), physiological evidence supports a rate code model, even though the cellular mechanisms require continued investigation. Large populations of ITD sensitive cells recorded from the MSO in gerbil (Brand et al., 2002; 
Pecka et al., 2008) and IC of guinea pig (McAlpine et al., 2001) revealed ITD curves that have peak discharge rates restricted to delays falling outside the ecological window of both

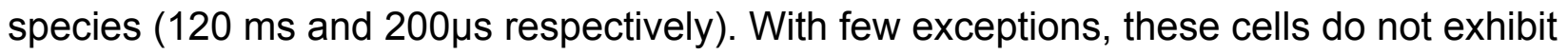
properties that are consistent with a place coding mechanism. Nonetheless, reliance on a rate code poses challenges to position the ITD curve along the ITD axis so that the steepest slope passes through the ecological range of hearing.

As suggested, some MSO neurons in large-headed mammals could employ a rate code, but this poses challenges to shift the ITD peak to larger values than is necessary for small-headed animals. In existing models, a single source of inhibition can achieve shifts of about $200 \mu \mathrm{s}$, which is sufficient for a gerbil or guinea pig. However, at lower frequencies, which are within the audible spectrum of large-headed animals, spike times are more distributed in time so that ITD peaks are not as sharp and slopes are not as steep as they are at higher frequencies. Not only must ITD peaks be shifted further, in cats to at least $400 \mu \mathrm{s}$, but additional shifts may be necessary so that the rounded peak of the ITD curve is outside the ecological range. At $250 \mathrm{~Hz}$ in our model, addition of a second inhibitory input offered great flexibility in the ability of the neural circuit to manipulate the ITD curve. Indeed, we could position the ITD peak at contralateral edge of the ecological range (shifted by $+400 \mu \mathrm{s}$ ), and the trough of the ITD curve at the ipsilateral edge of ecological range (near $-400 \mu \mathrm{s}$, Fig. 310).

At acoustic frequencies near the high frequency limit for ITD sensitivity, neural activity is concentrated in less than one-half of the stimulus period and does not extend across the entire ecological range of hearing (see McAlpine et al. (2001) for an example of frequency dependent ITD curve width). This situation applies to both large and small-headed animals. Changes in rate, then, must be positioned precisely to cover at least a significant portion of the contralateral hemifield in space to contribute to a population average of firing rate. Individual cells, then, will not represent all locations in space.

The limits of a rate code are evident in the processing of low and high frequencies. First, high frequency ITD sensitive neurons have narrow ITD curves that occupy a small region of the ecological range. Second, despite the proof of concept demonstrated in our model, physiological evidence in cat and dog show that ITD curves are not broad at low frequencies but narrow and only occupy a small portion of the ecological window. Therefore 
any coding strategy of ITDs for large-headed mammals must deal with both high and low frequency ITD cells having peak and trough discharge rates that fall within the ecological range. As an alternative, ITD coding strategies near the limits of sound localization might utilize multiple neurons having maximum slopes at a range of ITDs alleviating ambiguities generated from having peak and trough discharge rates falling within the ecological range. I propose that large-headed mammals localize low frequency sound with a rate-place code which is organized such that an array of neurons have maximum peaks and thus slopes tuned to different locations in space which converge later at higher auditory centers generating an unambiguous rate code.

In this model, individual IC neurons receive convergent projections from a range of MSO neurons whose peaks are positioned across the broad range of ITD (Fig. 6.1). If the MSO population is weighted toward cells with peaks at the edge or outside the ecological range, then IC neurons would also have peak discharge rates outside the ecological window and a steep slope through the ecological range. In small-headed mammals summations of the output of MSO neurons utilizing a rate code would result in an even steeper rate code at the level of the IC. Physiological evidence in the MSO and IC supporting a rate code model in small-headed animals is undeniable; however, like the MSO, the IC of large headed mammals has yielded little conclusive evidence supporting either sound localization paradigms (Rose et al.,1968). Nevertheless, summation of MSO output by IC neurons can improve neural encoding of sound location and discrimination between nearby sources of sound for both large and small-headed animals. In the case of large-headed animals, a rateplace code would be converted to a population rate code.

We investigated inhibitory and excitatory interactions in the one of the few anatomically accurate MSO cell models to date; however, two dendrites extended roughly perpendicular (rostrally and caudally) to the section of the tissue and could not be completely reconstructed. Therefore, we simplified the morphology of our reconstructed MSO cell by clipping these dendrites at the soma of our model. In bipolar MSO models, each dendrite receives excitatory inputs that are temporally unimodal. Unilaterally these dendrites sum EPSPs non-linearly and function as current sinks that bilaterally narrow the coincidence detections window and thus improve ITD sensitivity (Agmon Snir 1998). In cat, some percentage (no data available) of bipolar MSO cells have dendrites extending in the rostral 
and caudal directions. These have not been well characterized morphologically (Schwartz, 1977) so inferences of function are difficult to make. Comparatively, the dendritic morphology of adult gerbils is simple (Rautenberg et al., 2009; Russell and Moore, 1999) as these neurons lack the complex tertiary dendritic tufts found in cat (Schwartz, 1977). Almost nothing is known about the innervation of rostral and caudal dendrites. It does seem that these dendrites would degrade ITD sensitivity, especially if excitatory terminals found on the proximal dendrites were capable of depolarizing the cell to threshold either singly or through coincidence with other inputs. On the other hand if these dendrites only received inputs that delivered excitation asynchronously and they were incapable of inducing an AP, then the resulting slight depolarization could, for example, increase low threshold $\mathrm{K}$ conductance, lower cell input resistance and time constant, and narrow the coincidence window. Future physiological studies and computational models will need to address the role that these rostrally and caudally extending dendrites have on ITD coding.

In summary, these data demonstrate that phase-locking of MNTB cells to low frequency stimuli is less precise than their excitatory inputs, GBCs. In contrast, phase-locking of MNTB cells to SAM tones in unanesthetized cat is stronger than has been observed in anesthetized GBC and MNTB recordings. Retrograde labeling shows an approximately equal number of MNTB and LNTB cells were labeled from BDA injections in the MSO and thus justifies the use of equal numbers of inhibitory inputs in computational models of the MSO in large-headed mammals. Topographic organization of pvLNTB hint at a caudal locus of cells innervating MSO cells across rostro-caudal locations. Tonotopic organization of labeled MNTB cells demonstrate that innervation of MSO cells by the MNTB is somewhat tonotopically organized and is supported by another study showing that high frequency MNTB cells do not innervate the MSO (Smith et al., 1998). These anatomical restrictions on inhibitory sources undoubtedly have consequences in the processing of low frequency sounds and combined with our physiological finding in regards to the precision of MNTB cells serve as a guide to further exploration of ITD coding in computational models. Regardless, these results indicate that ITD coding is modified by a second source of inhibition, namely the LNTB, as well as an unexpectedly broad phase-locked inhibition from the MNTB. 

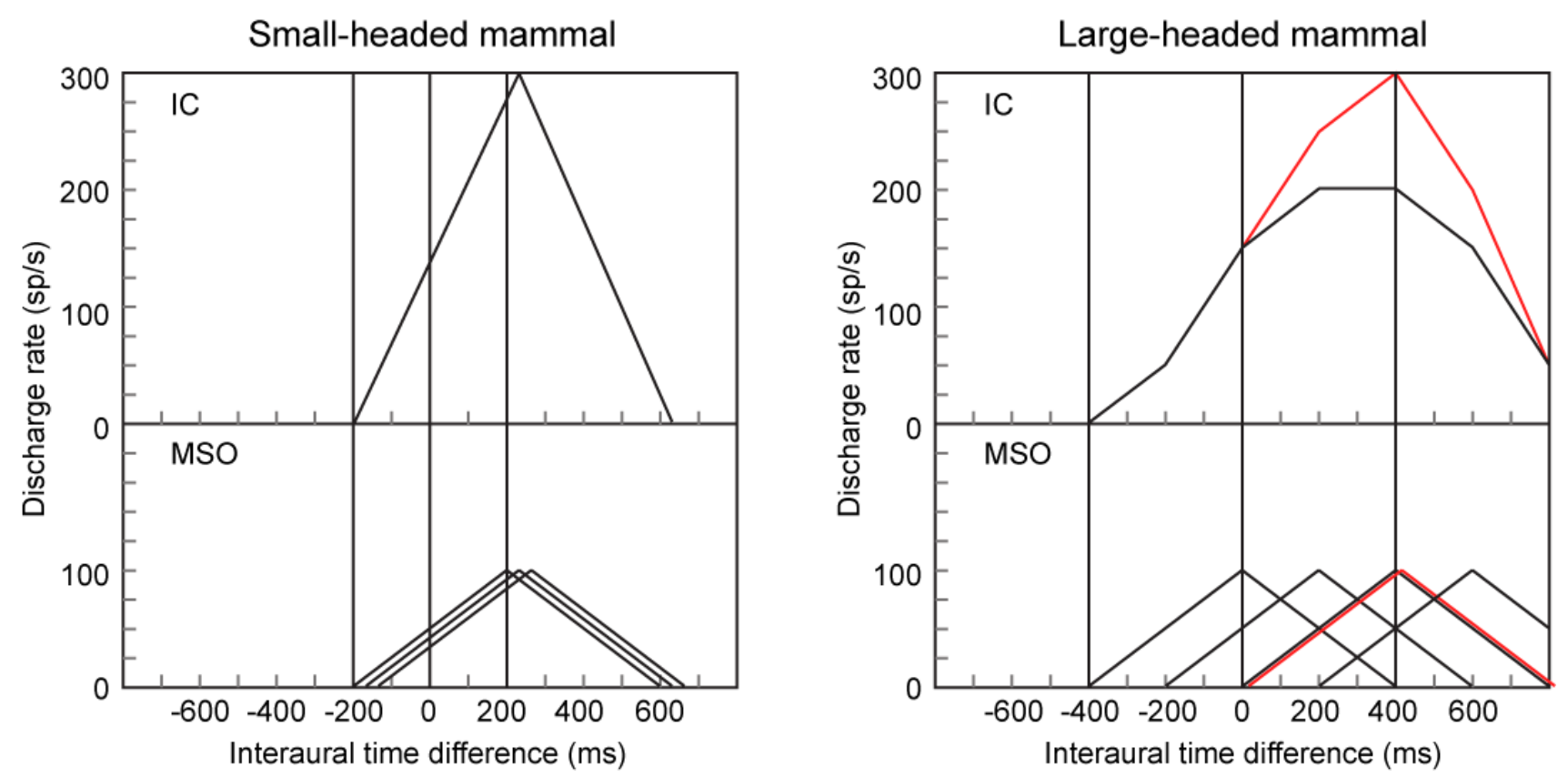

Figure 6-1.

Interaural temporal difference (ITD) curves of the MSO and IC. In small-headed animals discharge rates of MSO cell could be summed in the IC and result in an ITD curve having a peak discharge outside the ecological ITD window and a steep slope within the ecological range. In large-headed animals peak discharge rates of MSO cells are located at contralateral ITDs. At the IC summation of inputs leads to an ITD curve which has a peak discharge rate outside or near the ecological limits of ITD coding. 
7. Methods 
The following is a comprehensive review of the methods not employed in Chapter 3 (Journal of Neuroscience ms), but in the localization and acquisition of physiological responses of MNTB units summarized in Chapter 4.

Animals

Adult (>9 months) colony raised cats were purchased from Harlan of Indianapolis IN. The ears were examined to ensure that they were clean and that the tympanic membrane was intact and translucent (indicating no infection). Animals are housed at a WVU facility and the surgical techniques have been approved by the Institutional Animal Care and Use Committee of West Virginia University.

Anesthesia and Decerebration

Decerebrate cats were used to preserve the strength of inhibitory inputs that are located throughout the auditory nuclei of the brainstem (Evans and Nelson,1973). Cats were initially anesthetized with Xylazine $(1 \mathrm{mg} / \mathrm{Kg} \mathrm{IM})$ and Ketamine $(10 \mathrm{mg} / \mathrm{Kg})$. An areflexic state was maintained with additional injections of ketamine throughout the decerebration procedure. Cats were intubated by separation of the sternohyoid muscle and insertion of a cannula through a small hole in the trachea to maintain the airway. The head was clamped and the muscles overlying the parietal bones were transected and retracted rostrally. A small, $1.5 \mathrm{~cm}^{2}$ section of bone was removed covering the ectosylvian gyrus exposing the middle cerebral artery, which was then tied off at the edges and removed. The cortex and white matter were removed to reveal the hippocampus, which was then aspirated. Underlying the caudal hippocampus is the pulvinar, superior colliculus and brachium of the inferior colliculus. The rostral superior colliculus was then aspirated and the neuraxis transected, at which point anesthesia is no longer required. Gel foam was inserted into the opening along with saline saturated cotton balls.

The ear canals were exposed and the dorsal $2 / 3$ transected; dry cotton balls were placed in the canals to prevent accumulation of fluid in the ear canals. Two screw mounts were attached to the skull so that no pressure was exerted on the ear canals via the ear bars. One screw mount was affixed to the frontal bones overlying the sinuses and another more 
caudally at the rostral edges of the parietal bones. The head was positioned $20^{\circ}$ forward from the Horsley-Clark plane and the screw mounts were attached to rods of a stereotaxic frame. The screw mounts and stereotaxic frame provide a standard plane, which allowed us to reliably record from the MNTB.

\section{Setup}

After the animal was mounted in the stereotaxic frame and ear bars delicately placed in the canals, small holes were drilled in the bullae allowing pressure equalization and measurement of the animals hearing. A small electrode was inserted through this hole and rested on the inside of the bulla. Evoked potentials were recorded and the animals hearing assessed. Afterwards, polyethylene tubes $(30 \mathrm{~cm})$ were inserted. The skull caudal to the tentorium was opened from the lamboidal crest to slightly rostral to the foramen magnum and across the width of the brainstem exposing the cerebellum. The cerebellum was aspirated revealing the $4^{\text {th }}$ ventricle and the brainstem. When recording (see Fig. 7-1) from the brainstem, an agar 1\% saline solution was applied to minimize pulsation and preserve the preparation. Stimuli generated with TDT (Tucker Davis Technologies) hardware and software were delivered to the animal with custom belt amplifiers and speakers that have been attached to the ear bars. The stimulation hardware and software consisted of several components. Stimuli were constructed before hand utilizing TDT RPvds (Real-time Processor Visual Design Studio) with processors clock speeds at $100 \mathrm{kHz}$ allowing us to generate complex stimuli up to approximately $50 \mathrm{kHz}$ (Nyquist frequency). System calibration was performed every experiment using SigCal (TDT). Speaker performance was assessed and stored in a calibration file, which was subsequently loaded into the PA5s (programmable attenuators). After calibration, sound stimuli were presented to the animal using automated scripts in Brainware via the RX6 (Real-time Processor) and PA5s. After a cell was isolated and the CF determined, a response map was generated in software package Brainware (Jan Schnupp). Depending on the characteristics (monaural/binaural, threshold and CF) of the cell, an automated script controlled the presentation of stimuli while recording spike data for offline data analysis with custom Matlab programs. Unit discharges were amplified (Grass, AXON), filtered via TDT hardware PC1 and recorded via an RP2. Areas of interest were injected with biotinylated dextran amine (BDA) or fast green. Animals were maintained for 3-4 
days with daily injection of flocillin, an antibiotic, and injections every 4-6 hours of $60 \mathrm{ml}$ lactated ringer solution. Dependent on the level of involuntary movement, it was sometimes necessary to paralyze the animal with an injection of flaxidil (1cc/hour). While paralyzed, subjects were artificially respirated so that a $\mathrm{CO}_{2}$ level between 1.6 and $3.0 \%$ is maintained.

\section{Perfusion}

The animals were deeply anesthetized with pentobarbital $(40 \mathrm{mg} / \mathrm{kG})$ rendering them atonal and removed from the stereotaxic frame. The cat was placed on a down draft table and the limbs secured. An incision was made from the top of the sternum extending to the abdomen past the navel and the skin lateral to the incision was separated from the underlying musculature. The abdominal wall was opened laterally under the rib cage and another cut made anteroposterior. The diaphragm was cut away from the ribs and the animal was ventilated. The rib cage was cut bilaterally and the sternum pulled upward exposing the heart and lungs. The pericardium was opened and heart exposed; the right atrium and left ventricle were sliced and the descending aorta clamped. A cannula was inserted into the left ventricle and the tip inserted into the ascending aorta. The animal was perfused with saline (to remove blood cells) and then fixative. After perfusion, ice was placed on the head for one hour. The head was removed, and in experiments where the brain was blocked, placed in the stereotaxic frame where the brainstem was transected using a \#11 scalpel blade attached to a micromanipulator, which had been adjusted to the same angle as the electrode penetrations. The brain was removed from the skull, the dura peeled away and the brainstem placed in cryoprotectant for 2-3 days before freezing. The brainstem was sliced with a sliding microtome on a frozen stage. Tissue slices were rinsed, peroxidases blocked $(\mathrm{H} 2 \mathrm{O} 2$ and methanol) and membrane permeablized with the detergent triton. BDA injections were reacted with an avidin-biotin HRP macromolecular complex (ABC; Vectastain, Vector Labs, Burlingame, $C A$ ) and then visualized with DAB (3,3'-diaminobenzidine tetrahydrochloride) and nickel chloride. Avidin has multiple high affinity binding sites for biotin allowing it to bind the biotin-tagged HRP, the injected BDA. DAB is activated via peroxidase in the ABC/BDA complex and turns dark. After reacting, sections were mounted on slides with cresyl gel and later counterstained with cresyl violet for morphological analysis. 
Methods particular to Aim 2

Extracellular recordings were made in the MNTB with quartz and borosilicate pipettes. Stimulation of the MNTB consisted of an arrangement of auditory stimuli including noise bands, pure tone series, SAM tones, and binaural beats. These stimuli were used to determine a cell's characteristic frequency, response to binaural tones and sensitivity to interaural time differences. PSTHs were constructed from $60 \mathrm{~ms}$ pure tones at each cells CF. Response latencies were determined from click stimuli and pure tones (@CF @ 50 dB re: threshold). Response maps were generated from a series of pure tones whose frequency and attenuation varied about a cell's CF and threshold. SAM tones are described by the following equation: $s(t)=\left[1+m \sin \left(2 \pi f_{m} t\right)\right] \sin \left(2 \pi f_{c} t\right)$, where $f_{m}$ is the modulation frequency $m$ the modulation depth and $f_{c}$ the carrier frequency. VS were calculated from phase PSTHs generated from $1 \mathrm{~ms}$ SAM tones. Stimuli generated in TDT (systemll/3) software and hardware were presented through custom built amplifiers and speakers (Stax). Cell responses that were amplified $100 \mathrm{X}$ and filtered through $10 \mathrm{~Hz}-10 \mathrm{kHz}$ Grass amplifier were processed via TDT software and post-processed with custom Matlab software. 


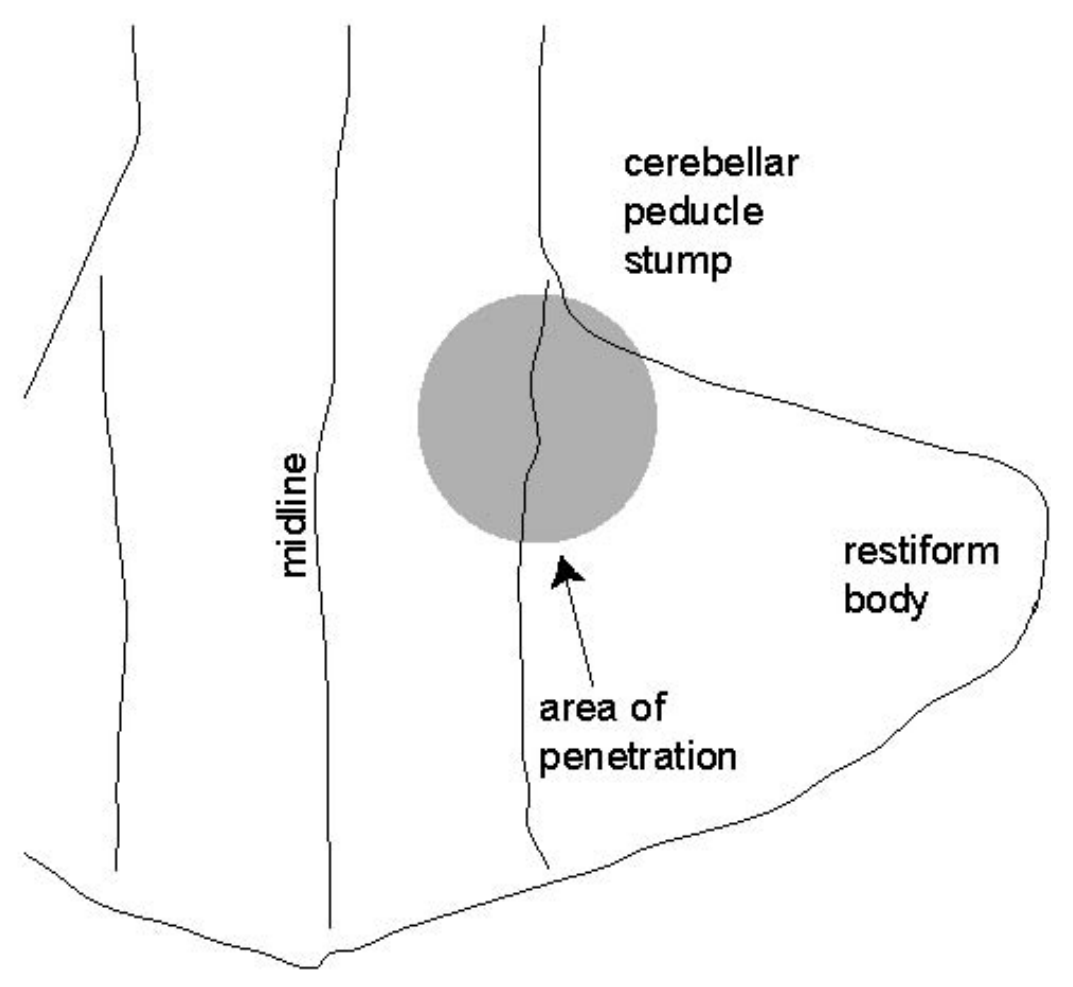

Figure 7-1.

Landmarks of the MNTB on the floor of the fourth ventricle. Stereotaxic of the MNTBThe floor of the fourth ventricle was exposed after the aspiration of the over hanging cerebellum. The penetration for the recording site (grey circle) was located parallel to the cerebellar peduncle and $.5-1 \mathrm{~mm}$ lateral of the midline. Subsequent penetrations were based on recordings made at the initial recording site and were positioned rostra-caudally and medial-laterally in .25 or .5 $\mathrm{mm}$ increments. 


\section{Literature Cited}

Adams JC (1983) Cytology of periolivary cells and the organization of their projections in the cat. J Comp Neurol 215:275-289.

Adams JC, Mugnaini E (1990) Immunocytochemical evidence for inhibitory and disinhibitory circuits in the superior olive. Hear Res 49:281-298.

Agmon-Snir H, Carr CE, Rinzel J (1998) The role of dendrites in auditory coincidence detection. Nature 393:268-272.

Anis NA, Berry SC, Burton NR, Lodge D (1983) The dissociative anaesthetics, ketamine and phencyclidine, selectively reduce excitation of central mammalian neurones by $\mathrm{N}$ methyl-aspartate. Br J Pharmacol 79:565-575.

Awatramani GB, Turecek R, Trussell LO (2004) Inhibitory control at a synaptic relay. J Neurosci 24:2643-2647.

Batra R, Kuwada S, Stanford TR (1989) Temporal coding of envelopes and their interaural delays in the inferior colliculus of the unanesthetized rabbit. J Neurophysiol 61:257268.

Batra R, Kuwada S, Fitzpatrick DC (1997) Sensitivity to interaural temporal disparities of lowand high-frequency neurons in the superior olivary complex. I. Heterogeneity of responses. Journal of Neurophysiology 78:1222-1236.

Beckius GE, Batra R, Oliver DL (1999) Axons from anteroventral cochlear nucleus that terminate in medial superior olive of cat: observations related to delay lines. J Neurosci 19:3146-3161.

Bernstein LR, Trahiotis C (2002) Enhancing sensitivity to interaural delays at high frequencies by using "transposed stimuli". J Acoust Soc Am 112:1026-1036.

Berrebi AS, Spirou GA (1998) PEP-19 immunoreactivity in the cochlear nucleus and superior olive of the cat. Neuroscience 83:535-554.

Blackburn CC, Sachs MB (1989) Classification of unit types in the anteroventral cochlear nucleus: PST histograms and regularity analysis. Journal of Neurophysiology 62:13031329.

Boudreau JC, Tsuchitani C (1968) Binaural interaction in the cat superior olive S segment. J Neurophysiol 31:442-454. 
Boudreau JC, Tsuchitani C (1970) Cat superior olive S-segment cell discharge to tonal stimulation. Contrib Sens Physiol 4:143-213.

Bourk TR (1976) Electrical Responses of Neural Units in the Anteroventral Cochlear Nucleus of the Cat. In: Department of Electrical Engineering and Computer Science: Massachusetts Institute of Technology.

Brand A, Behrend O, Marquardt T, McAlpine D, Grothe B (2002) Precise inhibition is essential for microsecond interaural time difference coding. Nature 417:543-547.

Brawer JR, Morest DK (1975) Relations between auditory nerve endings and cell types in the cat's anteroventral cochlear nucleus seen with the Golgi method and Nomarski optics. J Comp Neurol 160:491-506.

Brawer JR, Morest DK, Kane EC (1974) The neuronal architecture of the cochlear nucleus of the cat. J Comp Neurol 155:251-300.

Brownell WE (1975) Organization of the cat trapezoid body and the discharge characteristics of its fibers. Brain Res 94:413-433.

Brughera AR, Stutman ER, Carney LH, Colburn HS (1996) A Model with Excitation and Inhibition for Cells in the Medial Superior Olive. Auditory Neuroscience 2:219-233.

Brunso-Bechtold JK, Henkel CK, Linville C (1990) Synaptic organization in the adult ferret medial superior olive. J Comp Neurol 294:389-398.

Caird D, Klinke R (1983) Processing of binaural stimuli by cat superior olivary complex neurons. Exp Brain Res 52:385-399.

Cajal SRY (1909) Histologie du Systeme Nerveux de l'Homme et des Vertebres.

Cant NB (1991) Projections to the lateral and medial superior olivary nuclei from the spherical and globular bushy cells of the anteroventral cochlear nucleus. New York: Raven Press.

Cant NB, Morest DK (1979) Organization of the neurons in the anterior division of the anteroventral cochlear nucleus of the cat. Light-microscopic observations. Neuroscience 4:1909-1923.

Cant NB, Casseday JH (1986) Projections from the anteroventral cochlear nucleus to the lateral and medial superior olivary nuclei. J Comp Neurol 247:457-476. 
Cant NB, Hyson RL (1992) Projections from the lateral nucleus of the trapezoid body to the medial superior olivary nucleus in the gerbil. Hear Res 58:26-34.

Carr CE, Konishi M (1988) Axonal delay lines for time measurement in the owl's brainstem. Proc Natl Acad Sci U S A 85:8311-8315.

Chirila FV, Rowland KC, Thompson JM, Spirou GA (2007) Development of gerbil medial superior olive: integration of temporally delayed excitation and inhibition at physiological temperature. The Journal of Physiology 584:167-190.

Clark GM (1969a) Vesicle shape versus type of synapse in the nerve endings of the cat medial superior olive. Brain Res 15:548-551.

Clark GM (1969b) The ultrastructure of nerve endings in the medial superior olive of the cat. Brain Res 14:293-305.

Couchman K, Grothe B, Felmy F (2010) Medial superior olivary neurons receive surprisingly few excitatory and inhibitory inputs with balanced strength and short-term dynamics. J Neurosci 30:17111-17121.

Dasika VK, White JA, Colburn HS (2007) Simple models show the general advantages of dendrites in coincidence detection. J Neurophysiol 97:3449-3459.

Dodla R (2005) Well-Timed, Brief Inhibition Can Promote Spiking: Postinhibitory Facilitation. Journal of Neurophysiology 95:2664-2677.

Dreyer A, Delgutte B (2006) Phase Locking of Auditory-Nerve Fibers to the Envelopes of High-Frequency Sounds: Implications for Sound Localization. Journal of Neurophysiology 96:2327-2341.

Elverland $\mathrm{HH}$ (1978) Ascending and intrinsic projections of the superior olivary complex in the cat. Exp Brain Res 32:117-134.

Evans EF, Nelson PG (1973) The responses of single neurones in the cochlear nucleus of the cat as a function of their location and the anaesthetic state. Exp Brain Res 17:402427.

Fekete DM, Rouiller EM, Liberman MC, Ryugo DK (1984) The central projections of intracellularly labeled auditory nerve fibers in cats. J Comp Neurol 229:432-450.

Fitzpatrick DC, Batra R, Stanford TR, Kuwada S (1997) A neuronal population code for sound localization. Nature 388:871-874. 
Friauf E, Ostwald J (1988) Divergent projections of physiologically characterized rat ventral cochlear nucleus neurons as shown by intra-axonal injection of horseradish peroxidase. Exp Brain Res 73:263-284.

Frisina RD, Smith RL, Chamberlain SC (1985) Differential encoding of rapid changes in sound amplitude by second-order auditory neurons. Exp Brain Res 60:417-422.

Galambos R, Schwartzkopff J, Rupert A (1959) Microelectrode study of superior olivary nuclei. Am J Physiol 197:527-536.

Glattke TJ (1969) Unit responses of the cat cochlear nucleus to amplitude-modulated stimuli. J Acoust Soc Am 45:419-425.

Glendenning KK, Brunso-Bechtold JK, Thompson GC, Masterton RB (1981) Ascending auditory afferents to the nuclei of the lateral lemniscus. J Comp Neurol 197:673-703.

Glendenning KK, Hutson KA, Nudo RJ, Masterton RB (1985) Acoustic chiasm II: Anatomical basis of binaurality in lateral superior olive of cat. J Comp Neurol 232:261-285.

Godfrey DA, Kiang NY, Norris BE (1975) Single unit activity in the posteroventral cochlear nucleus of the cat. J Comp Neurol 162:247-268.

Goldberg JM, Brown PB (1969) Response of binaural neurons of dog superior olivary complex to dichotic tonal stimuli: some physiological mechanisms of sound localization. J Neurophysiol 32:613-636.

Goldberg JM, Brownell WE (1973) Discharge characteristics of neurons in anteroventral and dorsal cochlear nuclei of cat. Brain Res 64:35-54.

Griffin SJ (2005) Neural Sensitivity to Interaural Envelope Delays in the Inferior Colliculus of the Guinea Pig. Journal of Neurophysiology 93:3463-3478.

Guinan JJ, Li RY (1990) Signal processing in brainstem auditory neurons which receive giant endings (calyces of Held) in the medial nucleus of the trapezoid body of the cat. Hear Res 49:321-334.

Guinan JJ, Norris BE, Guinan SS (1972a) Single Auditory Units in the Superior Olivary Complex II: Locations of Unit Categories and Tonotopic Organization. J Neuroscience 4:147-166.

Guinan JJ, Norris BE, Guinan SS (1972b) Single auditory units in the superior olivary complex I: Responses to sounds and classifications based on physiological properties. J Neuroscience 4:101-120. 
Gundersen HJ, Jensen EB (1987) The efficiency of systematic sampling in stereology and its prediction. J Microsc 147:229-263.

Gundersen HJ, Jensen EB, Kiêu K, J N (1999) The efficiency of systematic sampling in stereology--reconsidered. J Microsc 193:199-211.

Hamann M, Billups B, Forsythe ID (2003) Non-calyceal excitatory inputs mediate low fidelity synaptic transmission in rat auditory brainstem slices. Eur J Neurosci 18:2899-2902.

Heffner RS, Heffner HE (1982) Hearing in the elephant (Elephas maximus): absolute sensitivity, frequency discrimination, and sound localization. J Comp Physiol Psychol 96:926-944.

Hermann J, Pecka M, von Gersdorff H, Grothe B, Klug A (2007) Synaptic transmission at the calyx of Held under in vivo like activity levels. J Neurophysiol 98:807-820.

Hines ML, Carnevale NT (1998) The Neuron Simulation Environment.26.

Jeffress LA (1948) A place theory of sound localization. J Comp Physiol Psychol 41:35-39.

Jercog PE, Svirskis G, Kotak VC, Sanes DH, Rinzel J (2010) Asymmetric excitatory synaptic dynamics underlie interaural time difference processing in the auditory system. Plos Biol 8:e1000406.

Johnson DH (1980) The relationship between spike rate and synchrony in responses of auditory-nerve fibers to single tones. J Acoust Soc Am 68:1115-1122.

Joris P, Yin TCT (2007) A matter of time: internal delays in binaural processing. Trends Neurosci 30:70-78.

Joris PX, Yin TC (1992) Responses to amplitude-modulated tones in the auditory nerve of the cat. J Acoust Soc Am 91:215-232.

Joris PX, Yin TC (1998) Envelope coding in the lateral superior olive. III. Comparison with afferent pathways. J Neurophysiol 79:253-269.

Joris PX, Smith PH, Yin TC (1994a) Enhancement of neural synchronization in the anteroventral cochlear nucleus. II. Responses in the tuning curve tail. Journal of Neurophysiology 71:1037-1051.

Joris PX, Carney LH, Smith PH, Yin TC (1994b) Enhancement of neural synchronization in the anteroventral cochlear nucleus. I. Responses to tones at the characteristic frequency. Journal of Neurophysiology 71:1022-1036. 
Kadner A, Berrebi AS (2008) Encoding of temporal features of auditory stimuli in the medial nucleus of the trapezoid body and superior paraolivary nucleus of the rat.

Neuroscience 151:868-887.

Kadner A, Kulesza RJ, Berrebi AS (2006) Neurons in the medial nucleus of the trapezoid body and superior paraolivary nucleus of the rat may play a role in sound duration coding. Journal of Neurophysiology 95:1499-1508.

Karino S, Smith PH, Yin TC, Joris PX (2011) Axonal branching patterns as sources of delay in the mammalian auditory brainstem: a re-examination. J Neurosci 31:3016-3031.

Kim DO, Sirianni JG, Chang SO (1990) Responses of DCN-PVCN neurons and auditory nerve fibers in unanesthetized decerebrate cats to $A M$ and pure tones: analysis with autocorrelation/power-spectrum. Hear Res 45:95-113.

Kiss A, Majorossy K (1983) Neuron morphology and synaptic architecture in the medial superior olivary nucleus. Light- and electron microscope studies in the cat. Exp Brain Res 52:315-327.

Koay G, Bitter KS, Heffner HE, Heffner RS (2002) Hearing in American leaf-nosed bats. I: Phyllostomus hastatus. Hear Res 171:96-102.

Kopp-Scheinpflug C, Lippe WR, Dorrscheidt GJ, Rubsamen R (2003a) The medial nucleus of the trapezoid body in the gerbil is more than a relay: comparison of pre- and postsynaptic activity. J Assoc Res Otolaryngol 4:1-23.

Kopp-Scheinpflug C, Tolnai S, Malmierca M, Rübsamen R (2008) The medial nucleus of the trapezoid body: Comparative physiology. Neuroscience.

Kopp-Scheinpflug C, Fuchs K, Lippe WR, Tempel BL, Rübsamen R (2003b) Decreased temporal precision of auditory signaling in Kcna1-null mice: an electrophysiological study in vivo. J Neurosci 23:9199-9207.

Kuwabara N, Zook JM (1991) Classification of the principal cells of the medial nucleus of the trapezoid body. J Comp Neurol 314:707-720.

Kuwabara N, Zook JM (1992) Projections to the medial superior olive from the medial and lateral nuclei of the trapezoid body in rodents and bats. J Comp Neurol 324:522-538.

Langner G, Schreiner CE (1988) Periodicity coding in the inferior colliculus of the cat. I. Neuronal mechanisms. J Neurophysiol 60:1799-1822. 
Leão RN, Leão RM, da Costa LF, Rock Levinson S, Walmsley B (2008) A novel role for MNTB neuron dendrites in regulating action potential amplitude and cell excitability during repetitive firing. Eur J Neurosci 27:3095-3108.

Leibold C (2010) Influence of inhibitory synaptic kinetics on the interaural time difference sensitivity in a linear model of binaural coincidence detection. J Acoust Soc Am 127:931-942.

Liberman MC (1978) Auditory-nerve response from cats raised in a low-noise chamber. J Acoust Soc Am 63:442-455.

Liberman MC (1991) Central projections of auditory-nerve fibers of differing spontaneous rate. I. Anteroventral cochlear nucleus. J Comp Neurol 313:240-258.

Liberman MC, Dodds LW, Pierce S (1990) Afferent and efferent innervation of the cat cochlea: quantitative analysis with light and electron microscopy. J Comp Neurol 301:443-460.

Lindsey BG (1975) Fine structure and distribution of axon terminals from the cochlear nucleus on neurons in the medial superior olivary nucleus of the cat. $\mathrm{J}$ Comp Neurol 160:81-103.

Lorente de No R (1933) Anatomy of the eighth nerve. I. The central projections of the nerve endings of the internal ear; III. General plan of structure of the primary cochlear nuclei. Laryngoscope:1-38\&327-350.

Lorente de No R (1981) The Primary Acoustic Nuclei. New York: Raven.

Mathews PJ, Jercog PE, Rinzel J, Scott LL, Golding NL (2010) Control of submillisecond synaptic timing in binaural coincidence detectors by K(v)1 channels. Nat Neurosci 13:601-609.

Mc Laughlin M, van der Heijden M, Joris PX (2008) How secure is in vivo synaptic transmission at the calyx of Held? J Neurosci 28:10206-10219.

McAlpine D, Jiang D, Palmer AR (2001) A neural code for low-frequency sound localization in mammals. Nat Neurosci 4:396-401.

Moore JK, Moore RY (1971) A comparative study of the superior olivary complex in the primate brain. Folia Primatol (Basel) 16:35-51. 
Morest DK (1968) The collateral system of the medial nucleus of the trapezoid body of the cat, its neuronal architecture and relation to the olivo-cochlear bundle. Brain Res 9:288-311.

Morest DK (1973) Auditory neurons of the brain stem. Adv Otorhinolaryngol 20:337-356.

Moushegian G, Rupert AL, Gidda JS (1975) Functional characteristics of superior olivary neurons to binaural stimuli. J Neurophysiol 38:1037-1048.

Mugnaini E (1985) GABA neurons in the superficial layers of the rat dorsal cochlear nucleus: light and electron microscopic immunocytochemistry. J Comp Neurol 235:61-81.

Mugnaini E, Osen KK, Dahl AL, Friedrich VL, Jr., Korte G (1980) Fine structure of granule cells and related interneurons (termed Golgi cells) in the cochlear nuclear complex of cat, rat and mouse. J Neurocytol 9:537-570.

Muller M (1990) Quantitative comparison of frequency representation in the auditory brainstem nuclei of the gerbil, Pachyuromys duprasi. Exp Brain Res 81:140-149.

Oliver DL, Beckius GE, Bishop DC, Loftus WC, Batra R (2003) Topography of interaural temporal disparity coding in projections of medial superior olive to inferior colliculus. $J$ Neurosci 23:7438-7449.

Osen KK (1969) Cytoarchitecture of the cochlear nuclei in the cat. J Comp Neurol 136:453484.

Ostapoff EM, Benson CG, Saint Marie RL (1997) GABA- and glycine-immunoreactive projections from the superior olivary complex to the cochlear nucleus in guinea pig. $J$ Comp Neurol 381:500-512.

Paolini AG, FitzGerald JV, Burkitt AN, Clark GM (2001) Temporal processing from the auditory nerve to the medial nucleus of the trapezoid body in the rat. Hear Res 159:101-116.

Pecka M, Brand A, Behrend O, Grothe B (2008a) Interaural Time Difference Processing in the Mammalian Medial Superior Olive: The Role of Glycinergic Inhibition. Journal of Neuroscience 28:6914-6925.

Pecka M, Brand A, Behrend O, Grothe B (2008b) Interaural time difference processing in the mammalian medial superior olive: the role of glycinergic inhibition. J Neurosci 28:69146925. 
Perkins RE (1973) An electron microscopic study of synaptic organization in the medial superior olive of normal and experimental chinchillas. J Comp Neurol 148:387-415.

Pfeiffer RR (1966) Classification of response patterns of spike discharges for units in the cochlear nucleus: tone-burst stimulation. Experimental brain research Experimentelle Hirnforschung Expérimentation cérébrale 1:220-235.

Reyes AD, Rubel EW, Spain WJ (1996) In vitro analysis of optimal stimuli for phase-locking and time-delayed modulation of firing in avian nucleus laminaris neurons. J Neurosci 16:993-1007.

Rhode W (2008) Response patterns to sound associated with labeled globular/bushy cells in cat. Neuroscience 154:87-98.

Rhode WS, Smith PH (1985) Characteristics of tone-pip response patterns in relationship to spontaneous rate in cat auditory nerve fibers. Hear Res 18:159-168.

Rhode WS, Greenberg S (1994) Encoding of amplitude modulation in the cochlear nucleus of the cat. J Neurophysiol 71:1797-1825.

Rhode WS, Oertel D, Smith PH (1983) Physiological response properties of cells labeled intracellularly with horseradish peroxidase in cat ventral cochlear nucleus. J Comp Neurol 213:448-463.

Richter JA, Holtman JR, Jr. (1982) Barbiturates: their in vivo effects and potential biochemical mechanisms. Prog Neurobiol 18:275-319.

Roberts RC, Ribak CE (1987) GABAergic neurons and axon terminals in the brainstem auditory nuclei of the gerbil. J Comp Neurol 258:267-280.

Rose JE, Gross NB, Geisler CD, Hind JE (1966) Some neural mechanisms in the inferior colliculus of the cat which may be relevant to localization of a sound source. $\mathrm{J}$ Neurophysiol 29:288-314.

Rose JE, Brugge JF, Anderson DJ, Hind JE (1967) Phase-locked response to low-frequency tones in single auditory nerve fibers of the squirrel monkey. J Neurophysiol 30:769793.

Rothman JS, Manis PB (2003a) Kinetic analyses of three distinct potassium conductances in ventral cochlear nucleus neurons. J Neurophysiol 89:3083-3096.

Rothman JS, Manis PB (2003b) Differential expression of three distinct potassium currents in the ventral cochlear nucleus. J Neurophysiol 89:3070-3082. 
Rothman JS, Young ED, Manis PB (1993) Convergence of auditory nerve fibers onto bushy cells in the ventral cochlear nucleus: implications of a computational model. $\mathrm{J}$ Neurophysiol 70:2562-2583.

Rouiller EM, Ryugo DK (1984) Intracellular marking of physiologically characterized cells in the ventral cochlear nucleus of the cat. J Comp Neurol 225:167-186.

Russell FA, Moore DR (1999) Effects of unilateral cochlear removal on dendrites in the gerbil medial superior olivary nucleus. European Journal of Neuroscience 11:1379-1390.

Russell FA, Moore DR (2002) Ultrastructural transynaptic effects of unilateral cochlear ablation in the gerbil medial superior olive. Hear Res 173:43-61.

Scheibel ME, Scheibel AB (1974) Neuropil organization in the superior olive of the cat. Exp Neurol 43:339-348.

Schwartz IR (1977) Dendritic Arrangements in Cat Medial Superior Olive. Neuroscience 2:81$\&$.

Scott LL, Mathews PJ, Golding NL (2010) Perisomatic voltage-gated sodium channels actively maintain linear synaptic integration in principal neurons of the medial superior olive. J Neurosci 30:2039-2050.

Seidl AH, Rubel EW, Harris DM (2010) Mechanisms for Adjusting Interaural Time Differences to Achieve Binaural Coincidence Detection. Journal of Neuroscience 30:70-80.

Shofner WP, Young ED (1985) Excitatory/inhibitory response types in the cochlear nucleus: relationships to discharge patterns and responses to electrical stimulation of the auditory nerve. J Neurophysiol 54:917-939.

Slomianka L, West MJ (2005) Estimators of the precision of stereological estimates: an example based on the CA1 pyramidal cell layer of rats. Neuroscience 136:757-767.

Smith PH (1995) Structural and functional differences distinguish principal from nonprincipal cells in the guinea pig MSO slice. J Neurophysiol 73:1653-1667.

Smith PH, Joris PX, Yin TC (1993) Projections of physiologically characterized spherical bushy cell axons from the cochlear nucleus of the cat: evidence for delay lines to the medial superior olive. J Comp Neurol 331:245-260.

Smith PH, Joris PX, Yin TC (1998) Anatomy and physiology of principal cells of the medial nucleus of the trapezoid body (MNTB) of the cat. Journal of Neurophysiology 79:31273142. 
Smith PH, Joris PX, Carney LH, Yin TC (1991) Projections of physiologically characterized globular bushy cell axons from the cochlear nucleus of the cat. J Comp Neurol 304:387-407.

Sommer I, Lingenhohl K, Friauf E (1993) Principal cells of the rat medial nucleus of the trapezoid body: an intracellular in vivo study of their physiology and morphology. Exp Brain Res 95:223-239.

Spangler KM, Warr WB, Henkel CK (1985) The projections of principal cells of the medial nucleus of the trapezoid body in the cat. J Comp Neurol 238:249-262.

Spangler KM, Cant NB, Henkel CK, Farley GR, Warr WB (1987) Descending projections from the superior olivary complex to the cochlear nucleus of the cat. J Comp Neurol 259:452-465.

Spirou G, Rager J, Manis P (2005) Convergence of auditory-nerve fiber projections onto globular bushy cells. Neuroscience 136:843-863.

Spirou GA, Young ED (1991) Organization of dorsal cochlear nucleus type IV unit response maps and their relationship to activation by bandlimited noise. J Neurophysiol 66:1750-1768.

Spirou GA, Berrebi AS (1996) Organization of ventrolateral periolivary cells of the cat superior olive as revealed by PEP-19 immunocytochemistry and Nissl stain. J Comp Neurol 368:100-120.

Spirou GA, Berrebi AS (1997) Glycine immunoreactivity in the lateral nucleus of the trapezoid body of the cat. J Comp Neurol 383:473-488.

Spirou GA, Brownell WE, Zidanic M (1990) Recordings from cat trapezoid body and HRP labeling of globular bushy cell axons. Journal of Neurophysiology 63:1169-1190.

Spirou GA, Rowland KC, Berrebi AS (1998) Ultrastructure of neurons and large synaptic terminals in the lateral nucleus of the trapezoid body of the cat. J Comp Neurol 398:257-272.

Spirou GA, Chirila FV, von Gersdorff H, Manis PB (2008) Heterogeneous $\mathrm{Ca}(2+)$ influx along the adult calyx of held: A structural and computational study. Neuroscience 154:171185.

Spitzer MW, Semple MN (1995) Neurons sensitive to interaural phase disparity in gerbil superior olive: diverse monaural and temporal response properties. J Neurophysiol 73:1668-1690. 
Stotler WA (1953) An experimental study of the cells and connections of the superior olivary complex of the cat. J Comp Neurol 98:401-431.

Strutt JW (1876) On Waves. Philos Mag 5:257-279.

Strutt JW (1877) The Theory of Sound. London: Macmillan.

Svirskis G, Dodla R, Rinzel J (2003) Subthreshold outward currents enhance temporal integration in auditory neurons. Biological Cybernetics 89:333-340.

Tolbert LP, Morest DK, Yurgelun-Todd DA (1982) The neuronal architecture of the anteroventral cochlear nucleus of the cat in the region of the cochlear nerve root: horseradish peroxidase labelling of identified cell types. Neuroscience 7:3031-3052.

Tolnai S, Hernandez O, Englitz B, Rübsamen R, Malmierca MS (2008) The medial nucleus of the trapezoid body in rat: spectral and temporal properties vary with anatomical location of the units. Eur J Neurosci 27:2587-2598.

Tsuchitani C (1977) Functional organization of lateral cell groups of cat superior olivary complex. J Neurophysiol 40:296-318.

Tsuchitani C (1994) The brain stem evoked response and medial nucleus of the trapezoid body. Otolaryngol Head Neck Surg 110:84-92.

Tsuchitani C (1997) Input from the medial nucleus of trapezoid body to an interaural level detector. Hear Res 105:211-224.

Tzounopoulos T, Kim Y, Oertel D, Trussell LO (2004) Cell-specific, spike timing-dependent plasticities in the dorsal cochlear nucleus. Nat Neurosci 7:719-725.

Uchizono K (1965) Characteristics of excitatory and inhibitory synapses in the central nervous system of the cat. Nature 207:642-643.

van Noort J (1969) The anatomical basis for frequency analysis in the cochlear nuclear complex. Psychiatr Neurol Neurochir 72:109-114.

Warr WB (1969) Fiber degeneration following lesions in the posteroventral cochlear nucleus of the cat. Exp Neurol 23:140-155.

Warr WB (1972) Fiber degeneration following lesions in the multipolar and globular cell areas in the ventral cochlear nucleus of the cat. Brain Res 40:247-270. 
Weiss TF, Rose C (1988) A comparison of synchronization filters in different auditory receptor organs. Hear Res 33:175-179.

Werthat F, Alexandrova O, Grothe B, Koch U (2008) Experience-dependent refinement of the inhibitory axons projecting to the medial superior olive. Dev Neurobiol 68:1454-1462.

Wiegrebe L, Meddis R (2004) The representation of periodic sounds in simulated sustained chopper units of the ventral cochlear nucleus. J Acoust Soc Am 115:1207-1218.

Winkler C (1907) The central course of the Nervus octavus and its influence on motility. Amsterdam: Johannes Muller.

Yin TC, Chan JC (1990) Interaural time sensitivity in medial superior olive of cat. Journal of Neurophysiology 64:465-488.

Young ED, Brownell WE (1976) Responses to tones and noise of single cells in dorsal cochlear nucleus of unanesthetized cats. J Neurophysiol 39:282-300.

Young ED, Robert JM, Shofner WP (1988) Regularity and latency of units in ventral cochlear nucleus: implications for unit classification and generation of response properties. Journal of Neurophysiology 60:1-29.

Young SR, Rubel EW (1983) Frequency-specific projections of individual neurons in chick brainstem auditory nuclei. J Neurosci 3:1373-1378.

Zhou Y, Carney LH, Colburn HS (2005) A Model for Interaural Time Difference Sensitivity in the Medial Superior Olive: Interaction of Excitatory and Inhibitory Synaptic Inputs, Channel Dynamics, and Cellular Morphology. Journal of Neuroscience 25:3046-3058. 
Jesse M. Thompson

\section{Curriculum vitae}

Address:

656 Afton St.

Morgantown, WV 26505

3046298422

thompson.jesse@gmail.com

Education:

West Virginia University College of Engineering and Mineral Resources

B.S., Computer Engineering 2001

West Virginia University School of Medicine

Ph.D., Physiology

Doctoral Dissertation:

Role of Inhibition in Binaural Processing

Research Interests:

Neural Mechanisms underlying sound localization

Computational modeling of neurons

Teaching Experience:

Medical Pharmacology 761

Small group facilitator for 12 medical students

Mentoring Experience:

Summer undergrad student Mark Daniel

Publications:

Jesse Paper

Chirila FV, Rowland KC, Thompson JM, Spirou GA. Development of gerbil medial superior olive: integration of temporally delayed excitation and inhibition at physiological temperature. The Journal of Physiology. October 2007

Abstracts:

Thompson JM, Brown PB, Millecchia R, Spirou GA. Distribution of bilateral inhibitory projections to medial superior olive and their functional effects on temporal coding. Society for Neuroscience, 2009

Chirila FV, Rowland KC, Thompson JM, Spirou GA. Development of gerbil medical superior olive: integration of temporally delayed excitation and inhibition at physiological temperature. Society for Neuroscience, 2007 
Thompson JM, Millecchia R, Brown PB, Samson F, Spirou GA. Temporal coding by MNTB neurons in unanesthetized cat. Society for Neuroscience, 2006

Thompson JM, Samson F, Brown PB, Millecchia R, Spirou GA. Sound-evoked activity of MNTB neurons in unanesthetized cat. Society for Neuroscience, 2005

Thompson JM, Rowland KC, Spirou GA. Cellular basis for ITD sensitivity in the MSO.

ARO, 2004

Spirou GA, Rowland KC, Thompson JM. Potassium channels and neural inhibition shape sensitivity to interaural temporal disparities for sound localization. Society for Neuroscience, 2003

Spirou GA, Rowland KC, Thompson JM. Cellular Specializations for Sound Localization. $6^{\text {th }}$ IBRO World Congress of Neuroscience, 2003

Muller BS, Thompson JM, Berrebi AS, Wince TJ, Spirou GA. Innervation of Cat MSO. ARO, 2002. 